Microwave-mediated Claisen Rearrangement followed by Phenol Oxidation: a Simple Route to Naturally Occurring 1,4-

Benzoquinones. The First Syntheses of Verapliquinones A and B and Panicein A

Christopher J. Davis, Timothy E. Hurst, Aouregan M. Jacob and Christopher J. Moody

SUPPORTING INFORMATION

\begin{tabular}{|l|l|l|l|}
\hline H/C NMR Spectra 10a & S2-3 & H/C NMR Spectra 15 & S39-40 \\
\hline H NMR Spectra 11a & S4 & H/C NMR Spectra 16 & S41 \\
\hline H NMR Spectra 12a & S5 & H NMR Spectra 5/6 & S42 \\
\hline H/C NMR Spectra 1 & S6-7 & H/C NMR Spectra 17 & S43-44 \\
\hline H/C NMR Spectra 10b & S8-9 & H/C NMR Spectra carbonate of 17 & S45-46 \\
\hline H/C NMR Spectra 11b & S10-11 & H/C NMR Spectra 18 & S47-48 \\
\hline H NMR Spectra 12b & S12 & H/C NMR Spectra 19 & S49-50 \\
\hline H/C NMR Spectra 2 & S13-14 & H/C NMR Spectra 20 & S51-52 \\
\hline H/C NMR Spectra pentadec-1-en-3-ol & S15-16 & H/C NMR Spectra 7 & S53-54 \\
\hline H/C NMR Spectra 10c & S17-18 & H/C NMR Spectra ethyl 3-(3-furyl)propenoate & S55-56 \\
\hline H/C NMR Spectra 11c & S19-20 & H/C NMR Spectra ethyl 3-(3-furyl)propanoate & S57-58 \\
\hline H/C NMR Spectra 12c & S21-22 & H/C NMR Spectra 21 & S59-60 \\
\hline H/C NMR Spectra 3 & S23-24 & H/C NMR Spectra 22 & S61-62 \\
\hline H/C NMR Spectra heptadec-1-en-3-ol & S25-26 & H/C NMR Spectra 23 & S63-64 \\
\hline H/C NMR Spectra 10d & S27-28 & H/C NMR Spectra 24 & S65-66 \\
\hline H/C NMR Spectra 11d & S29-30 & H/C NMR Spectra 9 & S67-68 \\
\hline H/C NMR Spectra 12d & S31-32 & Pentadec-1-en-3-ol, heptadec-1-en-3-ol & S69 \\
\hline H/C NMR Spectra 4 & S33-34 & Compounds 13 and 17 & S70 \\
\hline H/C NMR Spectra 13 & S35-36 & (E)-ethyl 3-(3-furyl)propenoate, ethyl 3-(3-furyl)propanoate & S71 \\
\hline H/C NMR Spectra 14 & S37-38 & Compounds 21 and 22 & S72 \\
\hline
\end{tabular}


1-Allyloxy-2-methoxybenzene 10a

cjd-ph
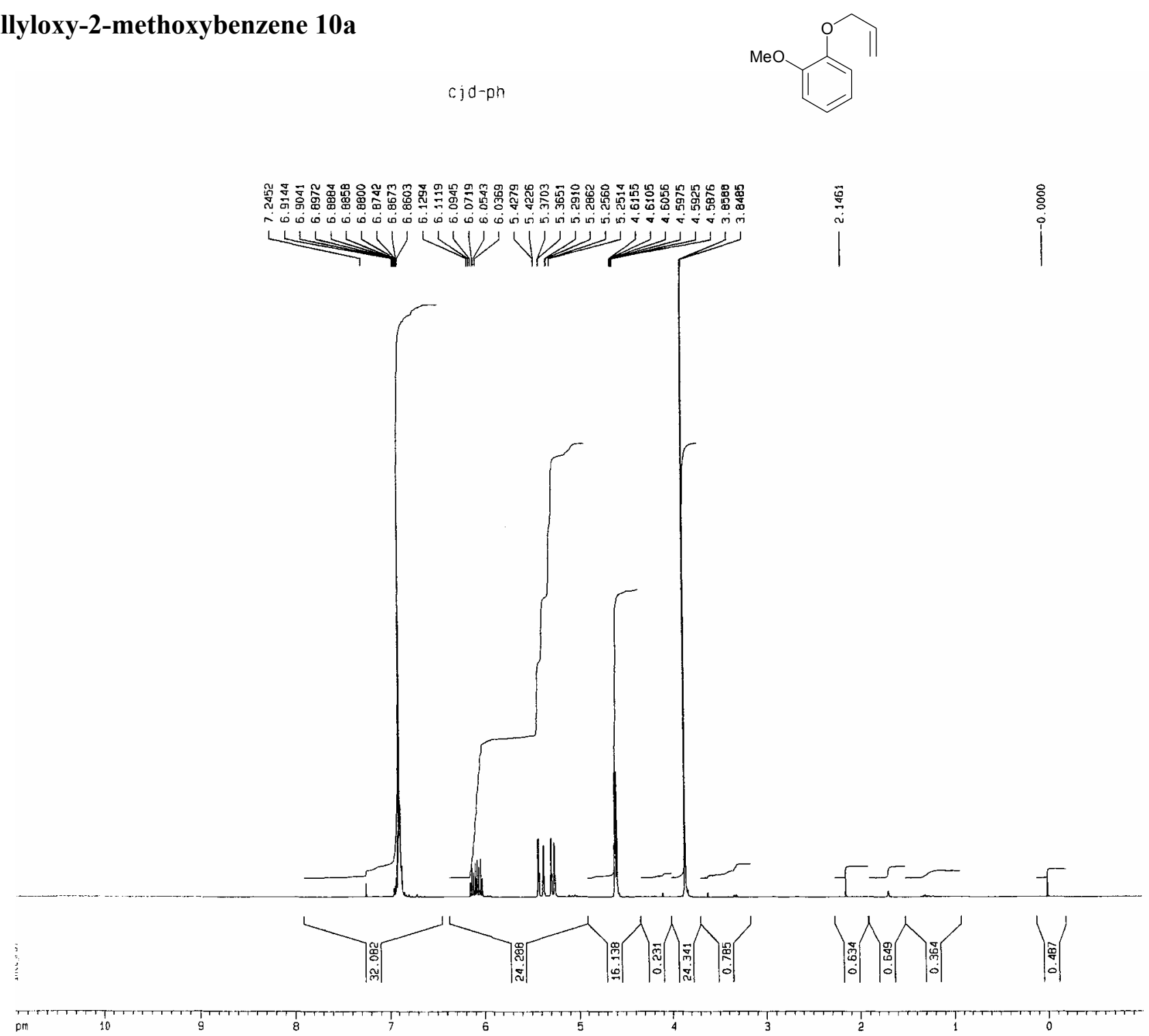

Current Data Parameters
NAME

EXPNO

F2- Acquisition Parameters

Date
Time

Iime
INSTRUM

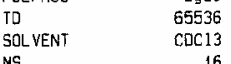

16
NS

$\begin{array}{ll}\text { SWH } & 6172,839 \mathrm{~Hz} \\ \text { FIDFES } & 0.094190 \mathrm{~Hz}\end{array}$

$\begin{array}{cc}\text { AQ } & 5.3004660 \mathrm{sec} \\ \text { AG } & 50.8\end{array}$

$\begin{array}{rr}\text { AG } & 50.8 \\ O K & 81.000 \text { usec } \\ \text { OK } & 6.00 \text { usec }\end{array}$

$\begin{array}{cc}\text { TE } & \begin{array}{c}300.0 \mathrm{~K} \\ 01\end{array} \\ 01.0000000 \mathrm{sec}\end{array}$

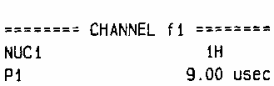

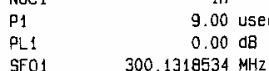

F2- Processing parameters
SI
SF

$\begin{array}{ll}\text { SI } & 32768 \\ \text { SF } & 300.1300105 \\ \text { MHOW } & \end{array}$

$\begin{array}{lc}\text { SFOW } & \text { EM } \\ \text { KSS } & 0 \\ \text { LB } & 0.30 \mathrm{~Hz}\end{array}$

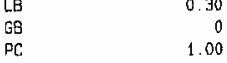

10 NMP plot parameters

$\begin{array}{ll}c X & 30.00 \mathrm{~cm} \\ \mathrm{CY} & 19.00 \mathrm{~cm}\end{array}$

$\begin{array}{lr}F_{1 P} & 11.000 \mathrm{pPm} \\ F_{10} & 3301.43 \mathrm{~Hz} \\ F_{22} & -1.000 \mathrm{~F}\end{array}$

$\begin{array}{ll}-1.000 \mathrm{pprm} \\ \mathrm{Fr} & -300.13 \mathrm{~Hz}\end{array}$

$\begin{array}{lr}\text { PPMCM } & 0.40000 \mathrm{ppm} / \mathrm{Cm} \\ \mathrm{HZCM} & 120.05200 \mathrm{~Hz} / \mathrm{cm}\end{array}$ 
cjd-ph
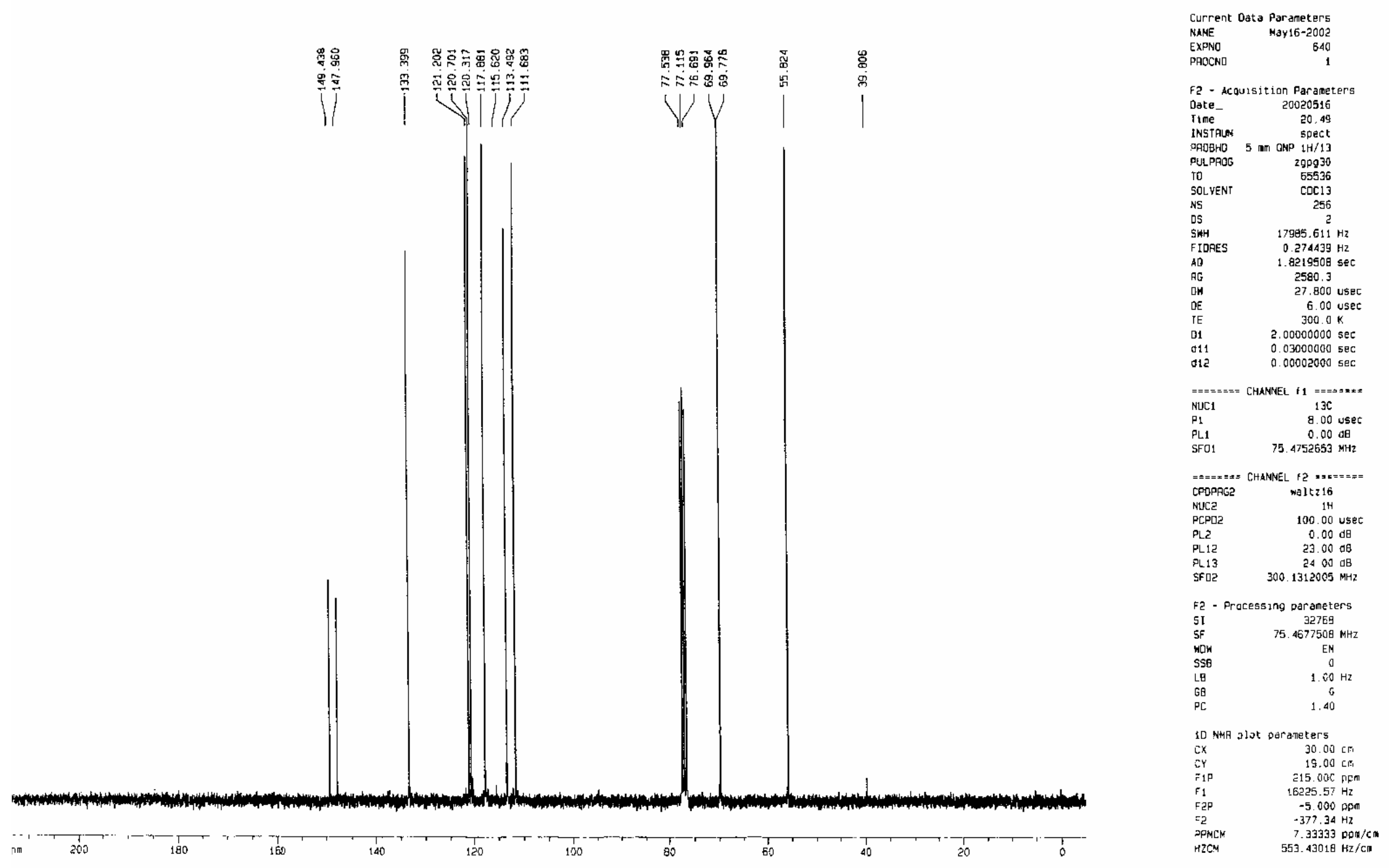


\section{2-Allyl-6-methoxyphenol 11a}

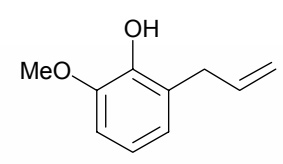

โปอ502月อ
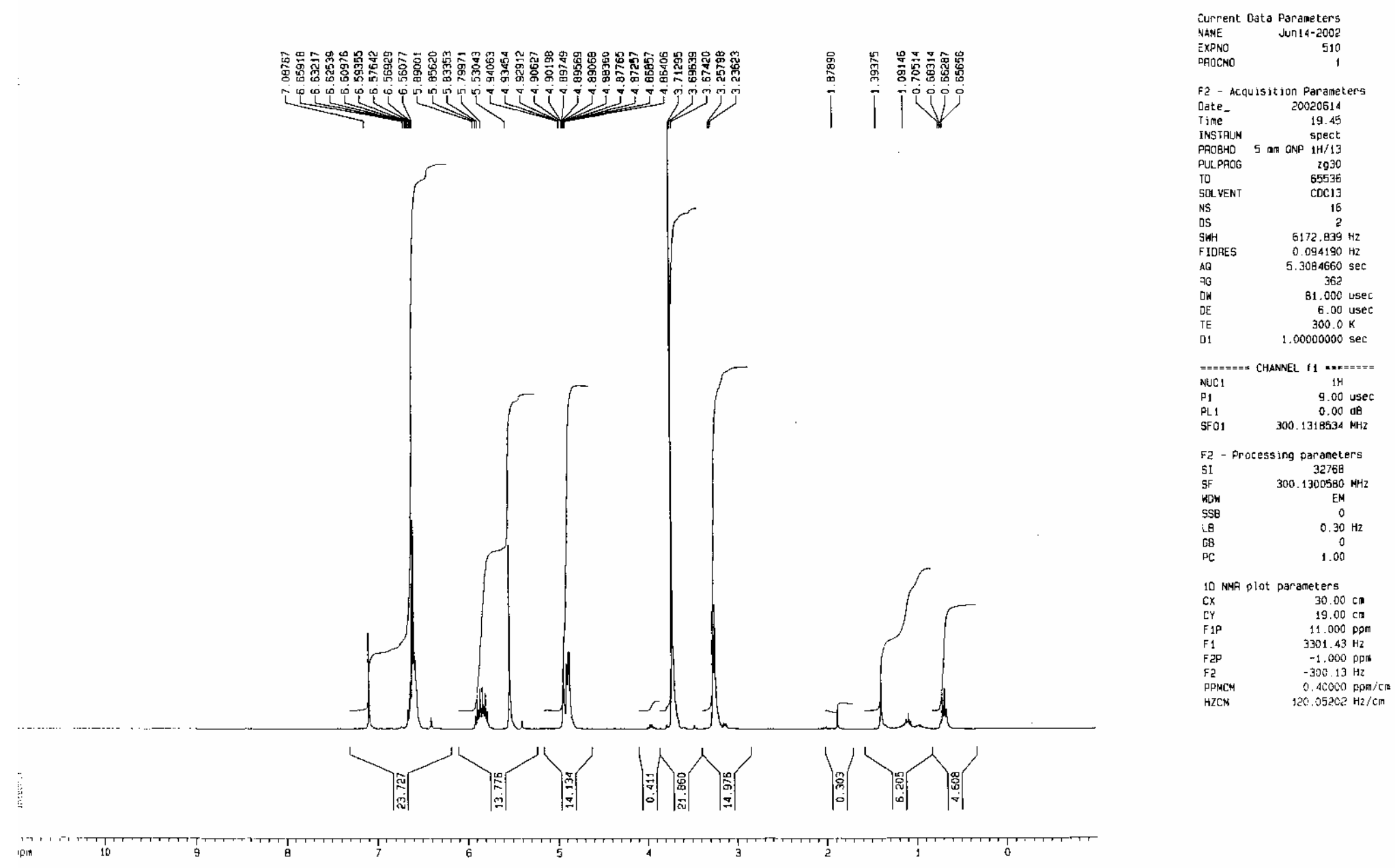
6-Methoxy-2-propylphenol 12a

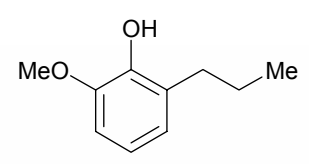

cj d524
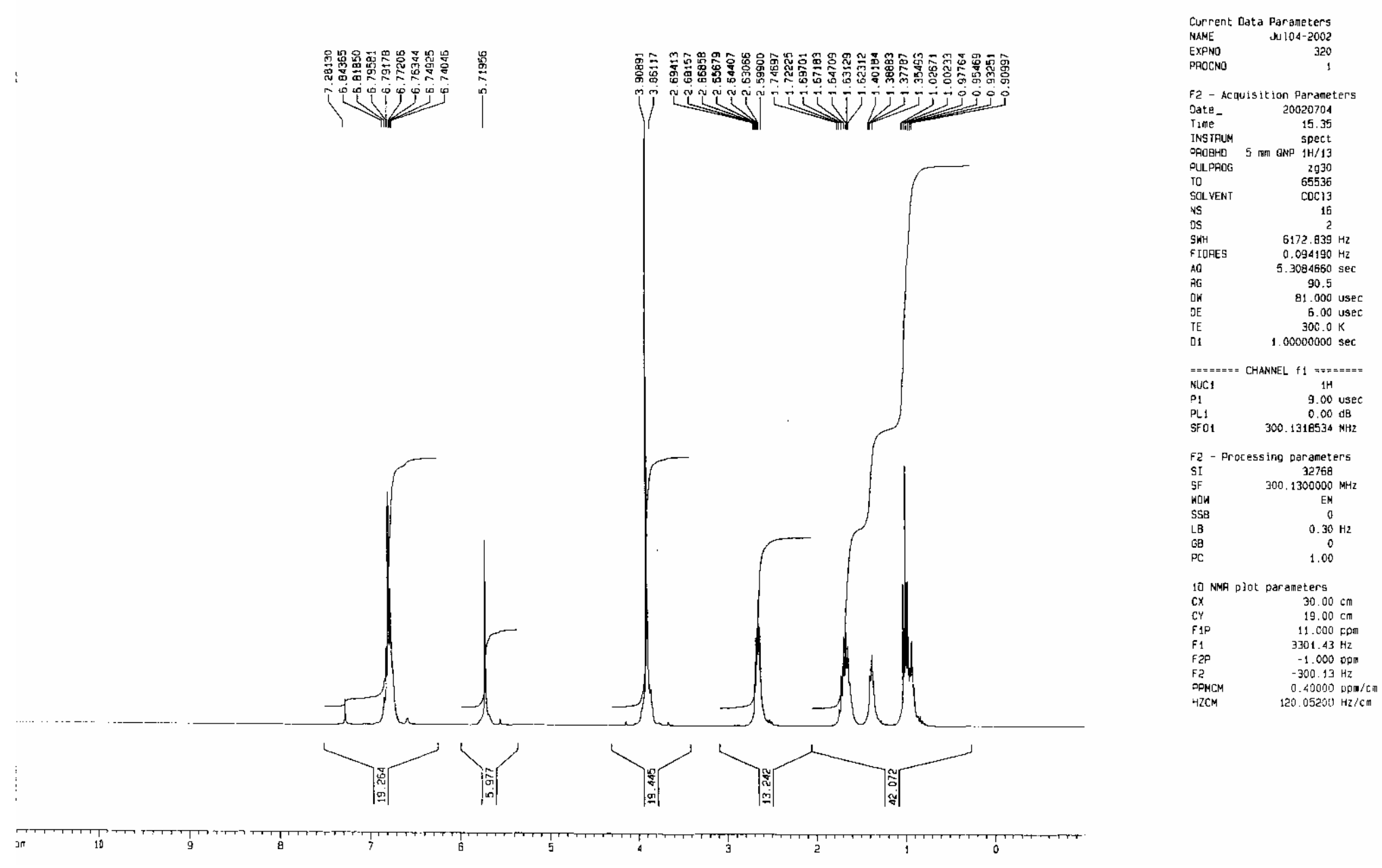
2-Methoxy-6-propyl-1,4-benzoquinone 1

.itis

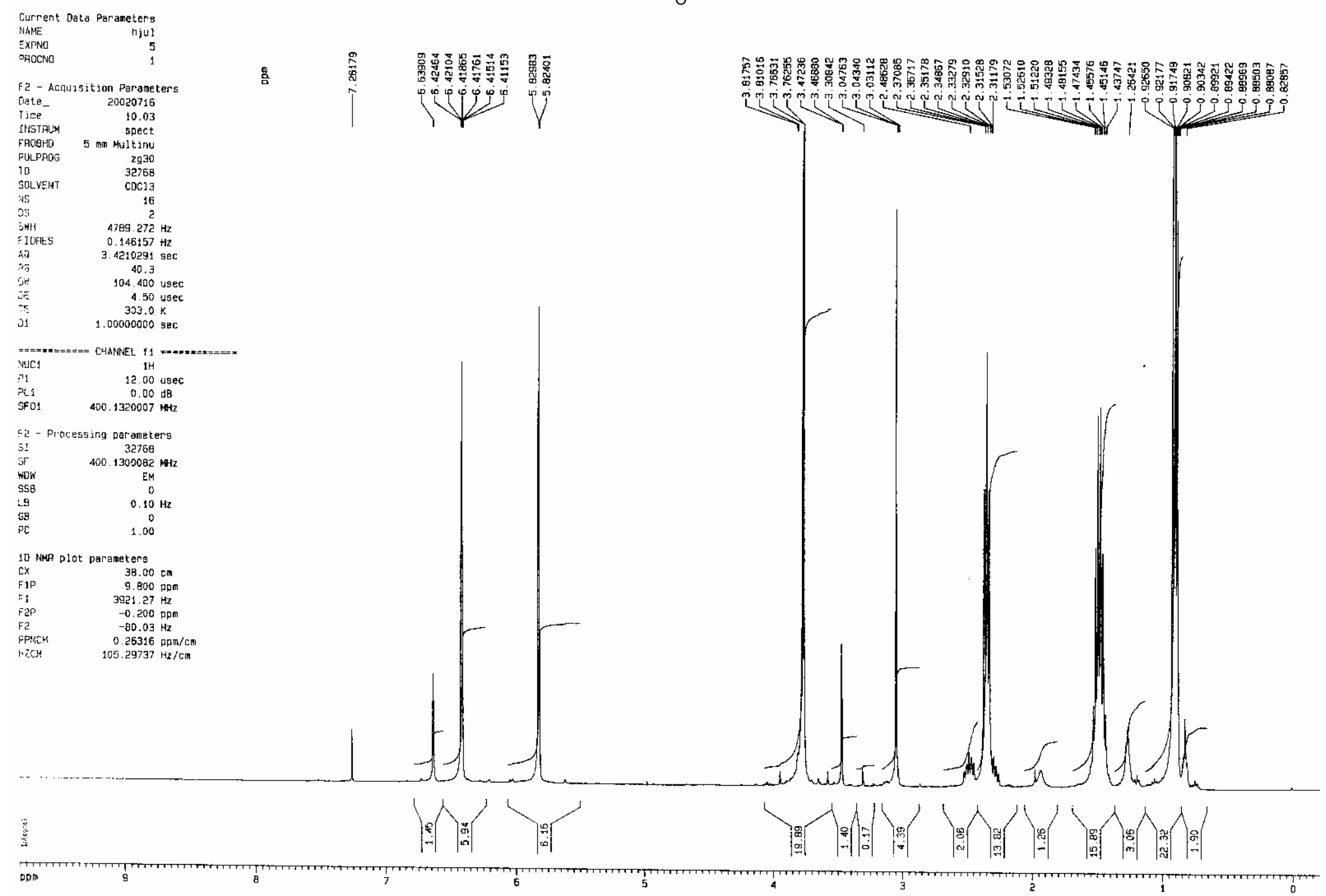


CJ10526
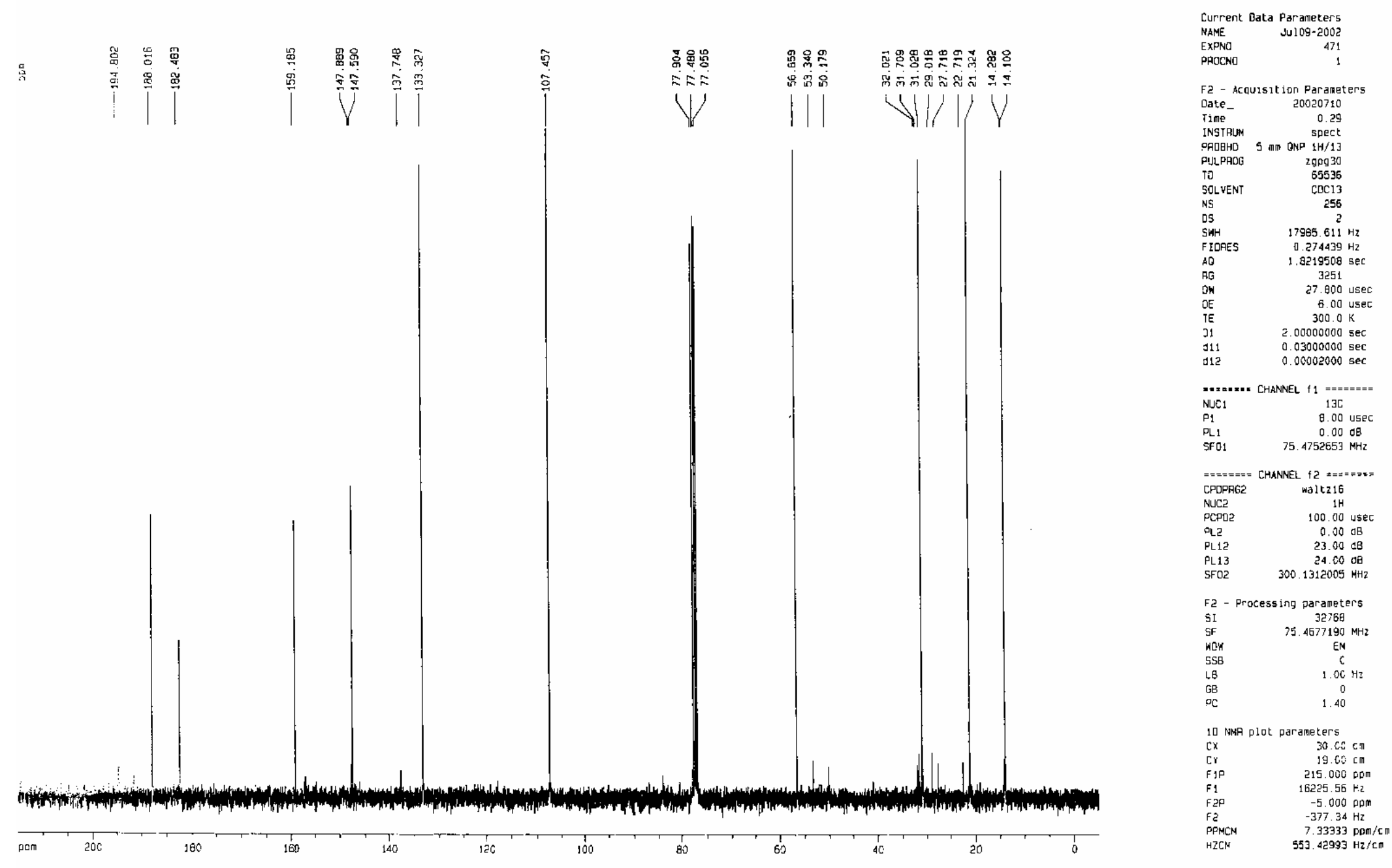
1-Methoxy-2-(pent-1-en-3-yloxy)benzene 10b

cja446e 1

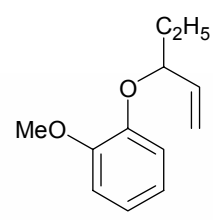

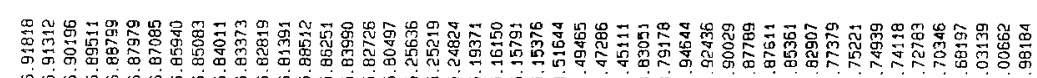

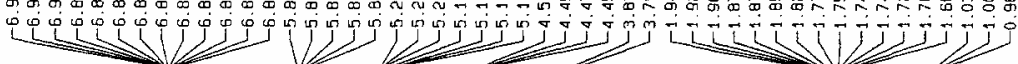

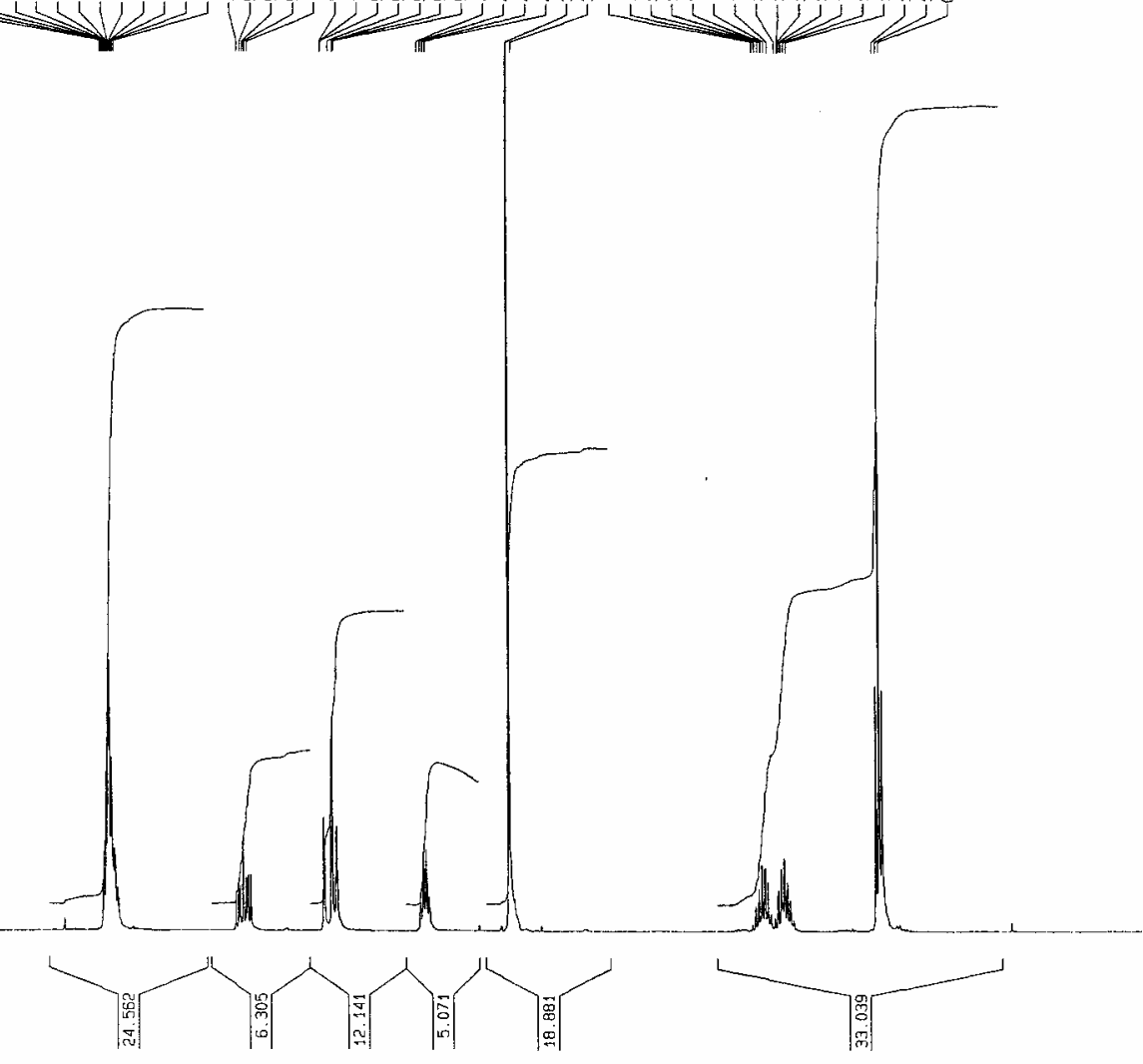

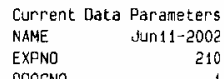

2. Acquisition farameters

Date- 20020611

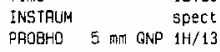

PULPPOG
IO

SOLVENT
NS

$\begin{array}{ll}\text { SHH } & 6172.939 \mathrm{~Hz} \\ \text { FHOAES } & 0.094190 \mathrm{~Hz}\end{array}$

$\begin{array}{ll}F \text { KOAES } & 0.094190 \mathrm{~Hz} \\ A B & 5.3084660 \mathrm{sec}\end{array}$

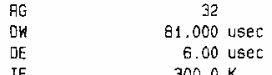

$\begin{array}{ll}\text { TE } & 3000 \mathrm{~K} \\ 01 & 1.00000000 \mathrm{sec}\end{array}$

$=\geq z= \pm==$ CHANNEL $f 1$
NUC1 $= \pm= \pm= \pm=$

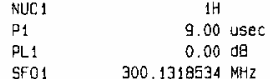

$\begin{array}{ll}\text { F2 - Processing par ameters } \\ \text { SI } & 32768 \\ \text { SF } & 300.1300184 \\ \text { SWHZ }\end{array}$

SF
HOW
SSI

$\begin{array}{cc}\text { LSP } & 0 \\ \text { LB } & 0.30 \mathrm{HZ} \\ \mathrm{GB} & 0\end{array}$

10 NMA p10L parameters

$\begin{array}{ll}\text { cX } & 30.00 \mathrm{~cm} \\ \mathrm{CY} & 19.00 \mathrm{~cm}\end{array}$

$\begin{array}{lr}\text { CY } & 19.00 \mathrm{~cm} \\ \mathrm{FIP} & 11.000 \mathrm{ppm} \\ \mathrm{Fi} & 3301.43 \mathrm{~Hz}\end{array}$

$\begin{array}{ll}F_{22} & -1.000 \mathrm{ppm} \\ F_{2} & -300.13 \mathrm{~Hz}\end{array}$

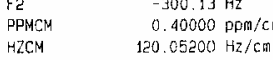


CJD446

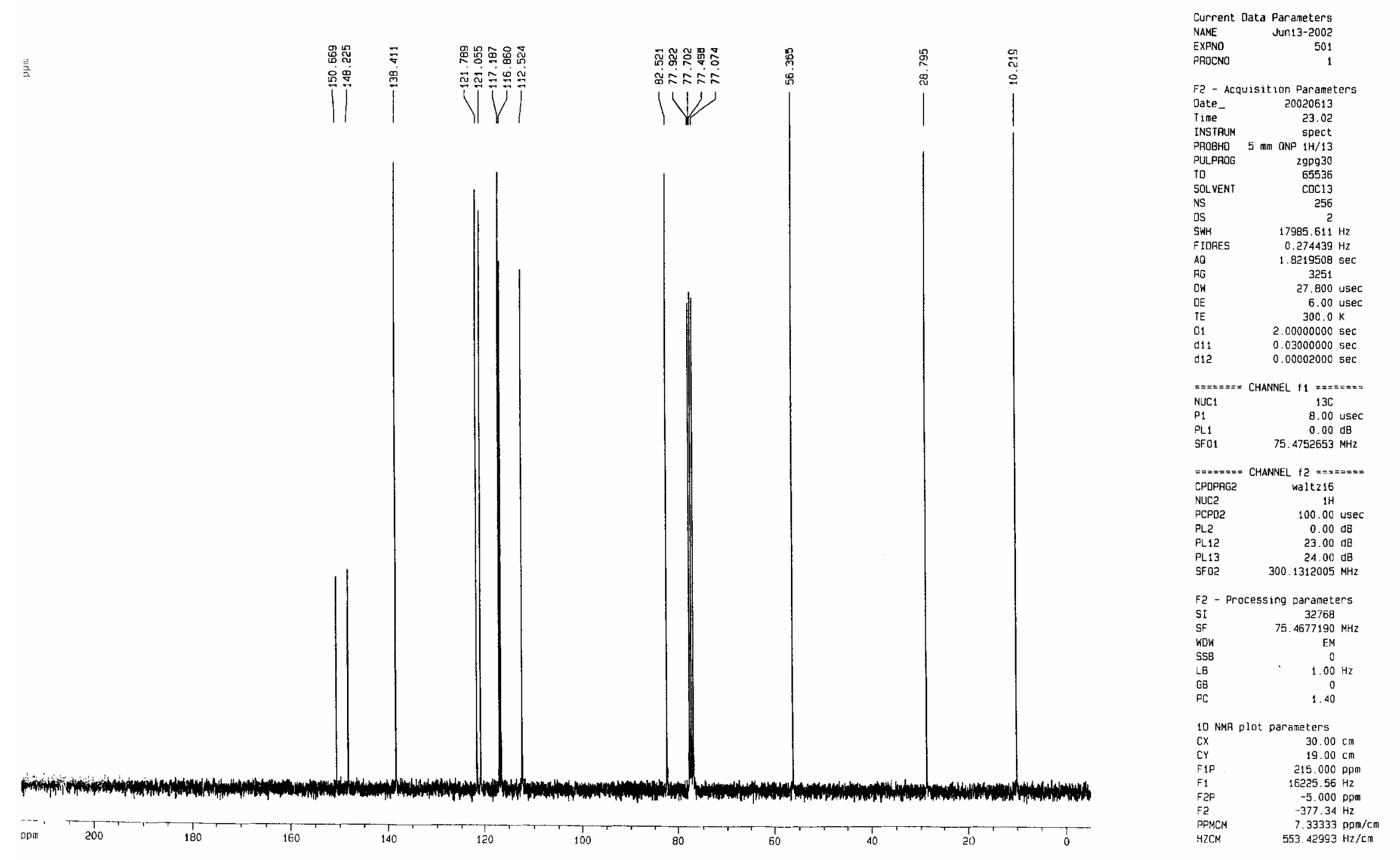


2-Methoxy-6-(pent-2-enyl)phenol 11b

cjo516
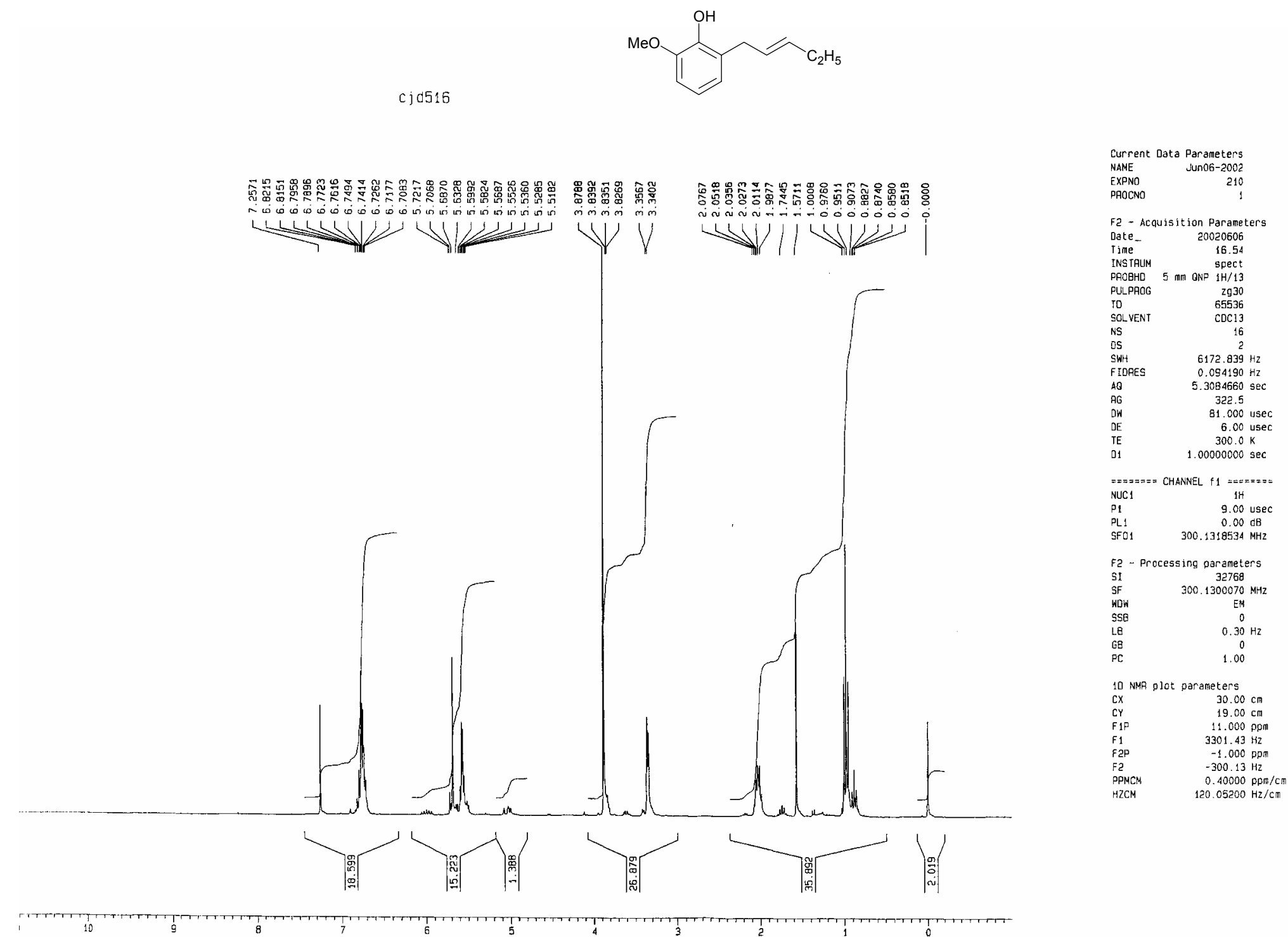
cjd516

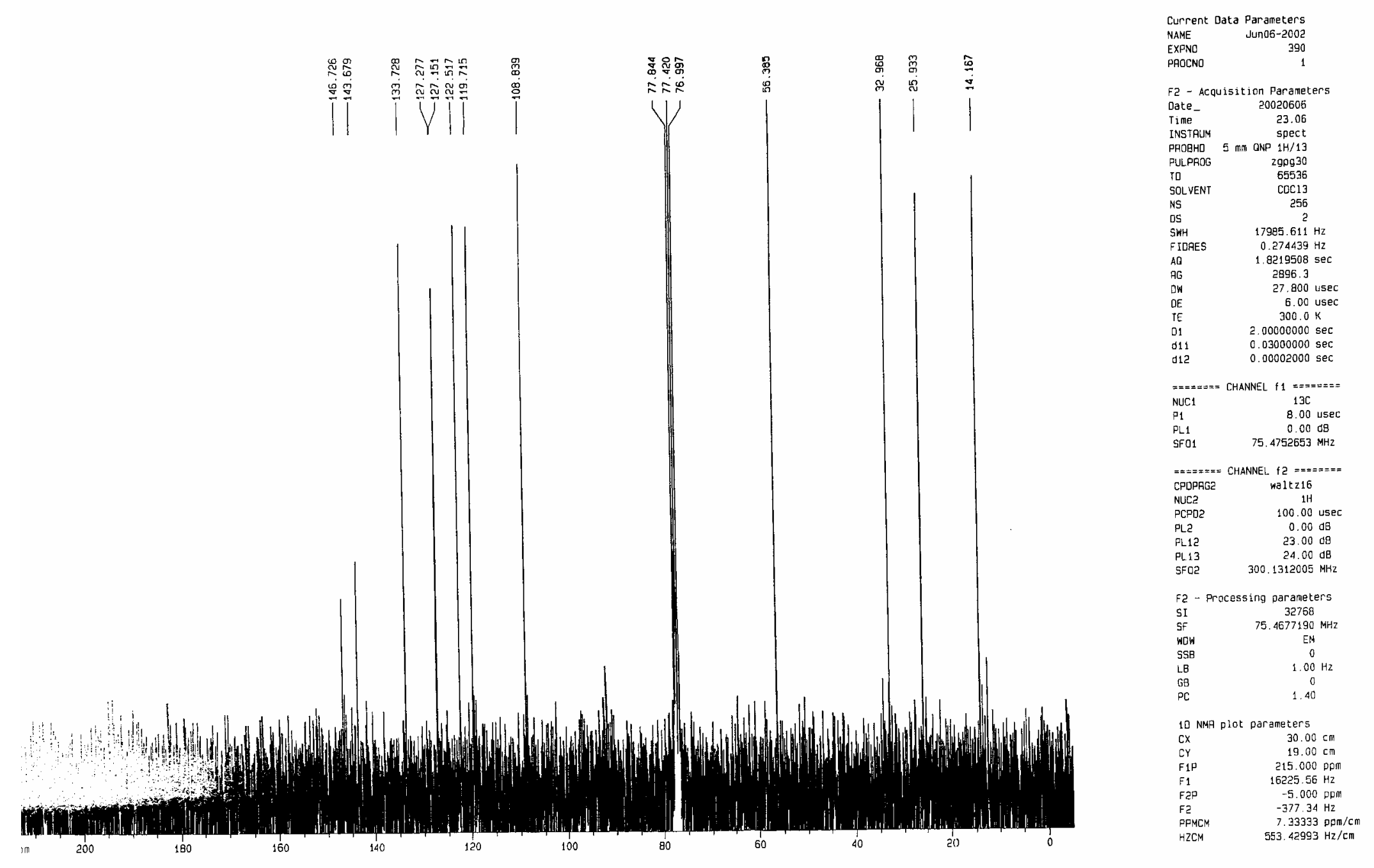


2-Methoxy-6-pentyl-phenol 12b

AMJ 520

MeO

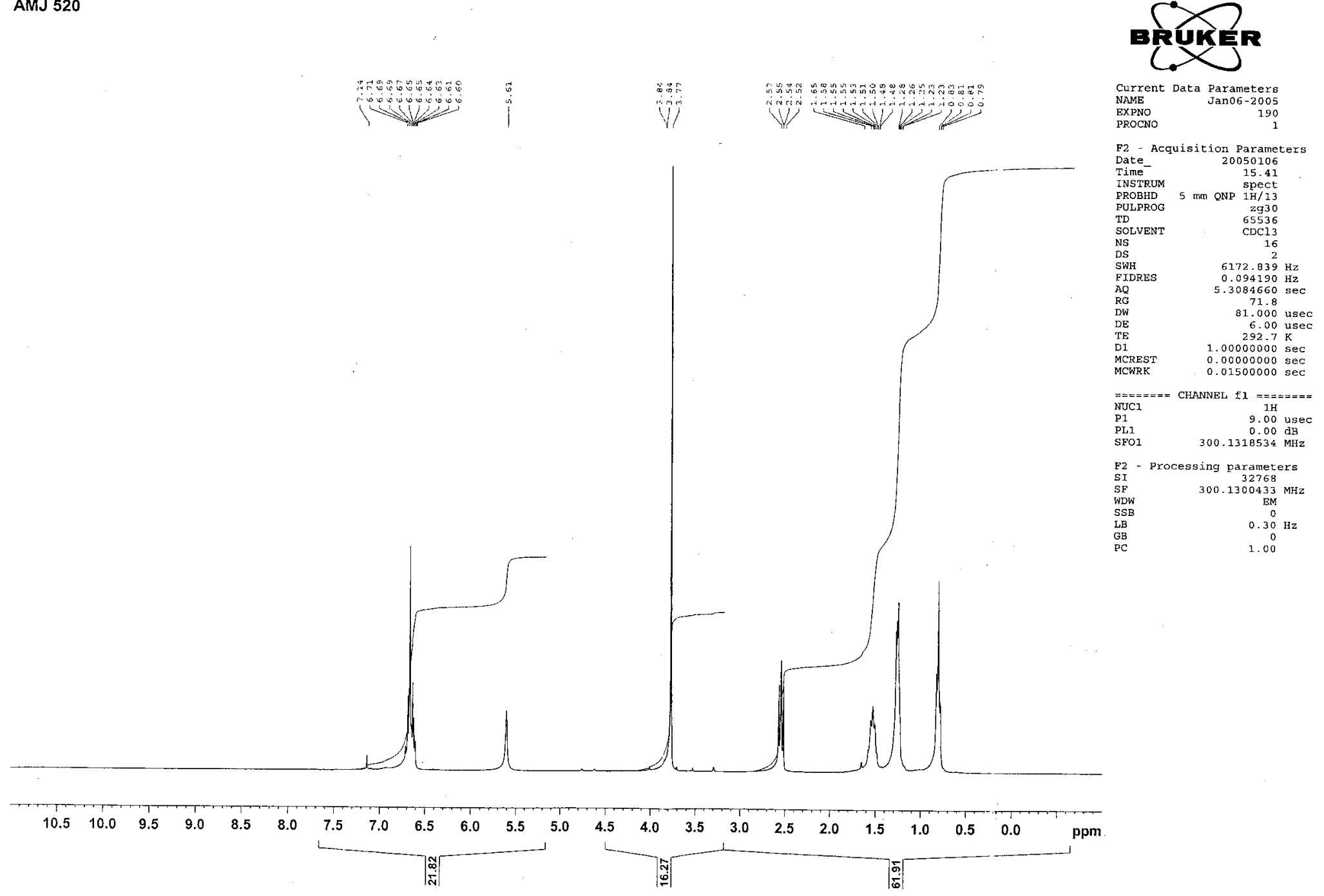

2- 
Methoxy-6-pentyl-1,4-benzoquinone (primin) 2

primin<smiles>CCCCC1=CC(=O)C=C(OC)C1=O</smiles>
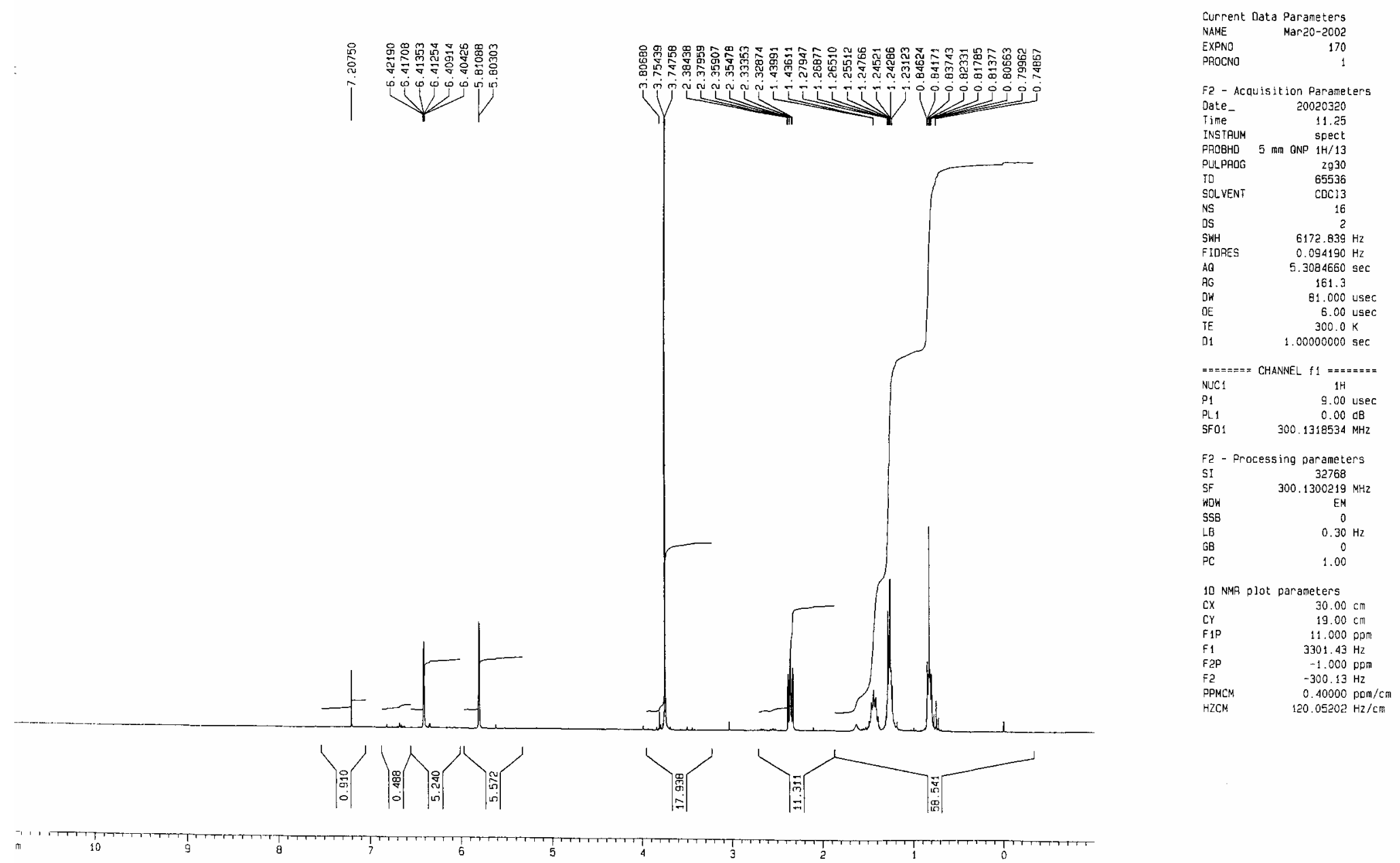
primin

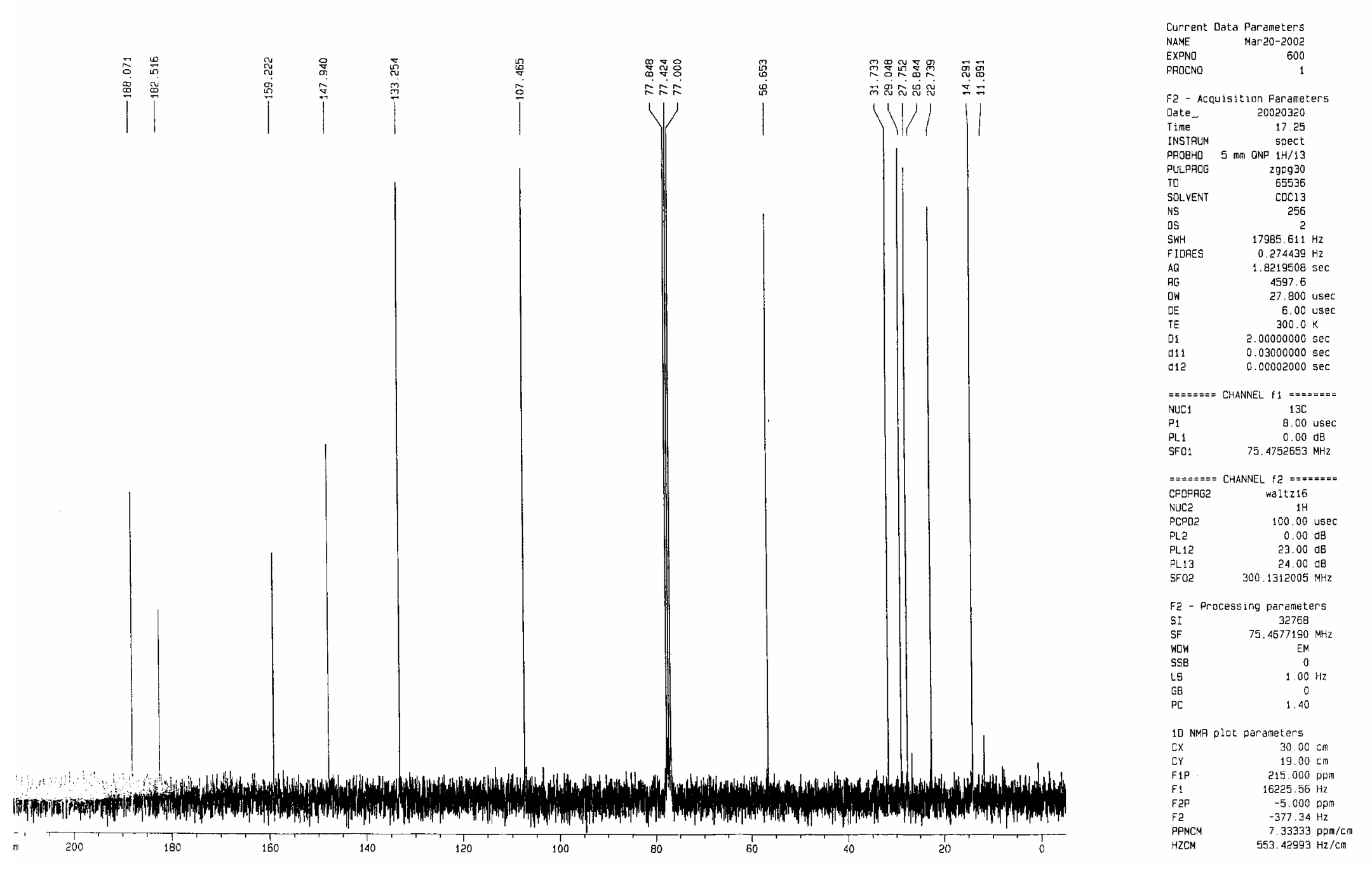




\section{Pentadec-1-en-3-ol}

sMu $: 10$

$$
\overbrace{12}^{\mathrm{C}_{12} \mathrm{H}_{25}}
$$

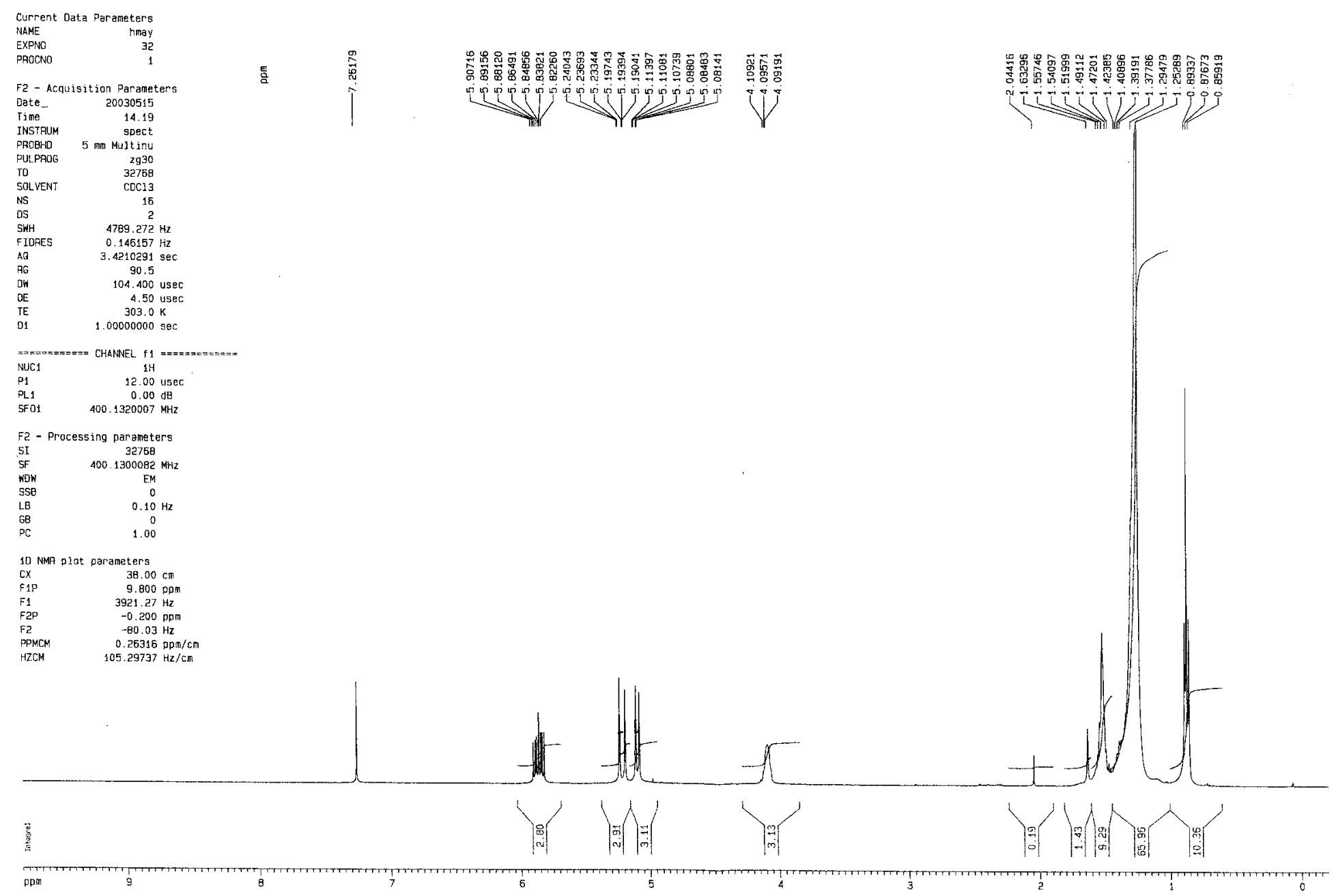




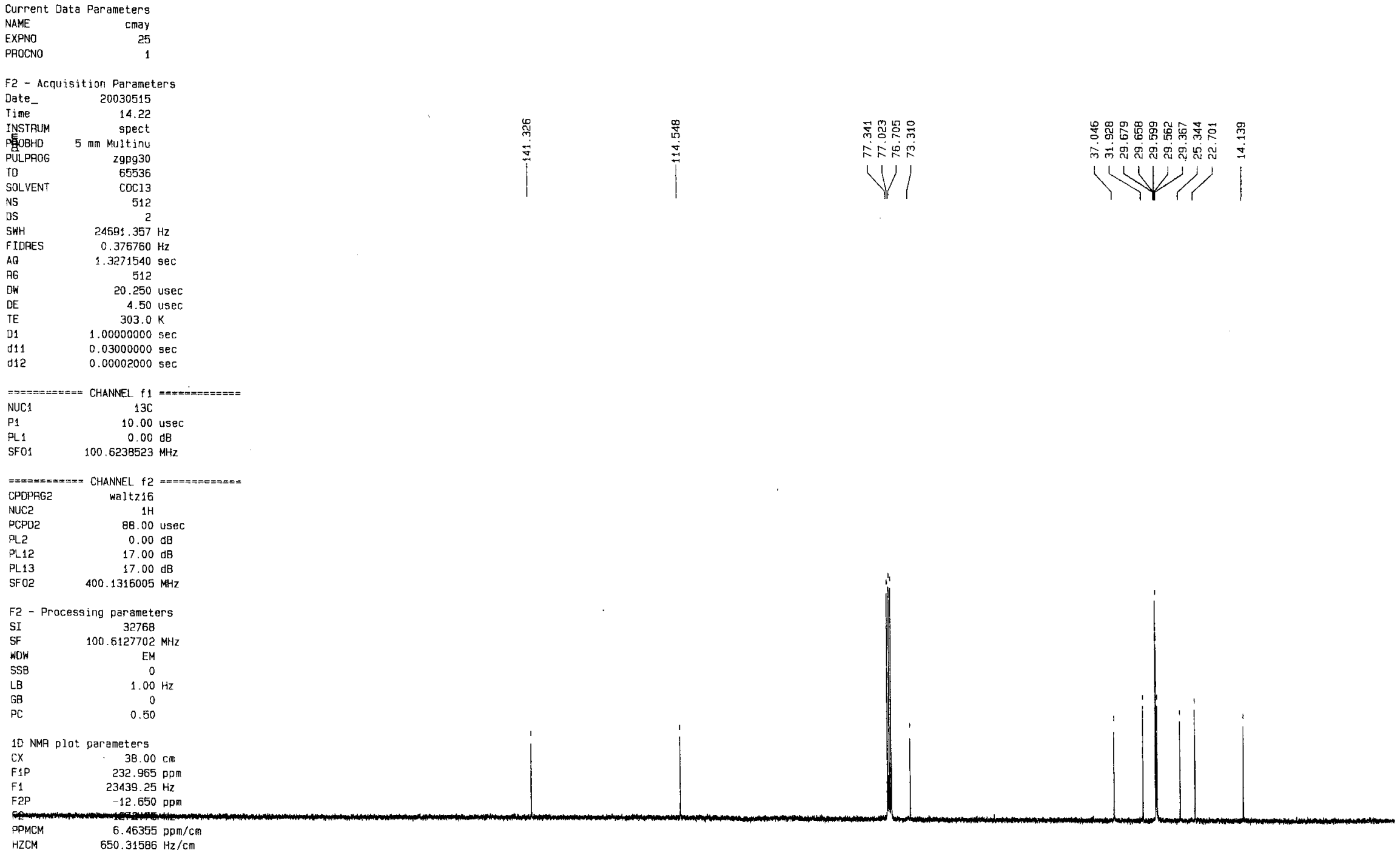


1-Methoxy-2-(pentadec-1-en-3-yloxy)benzene 10c

IMJ $22^{2}$

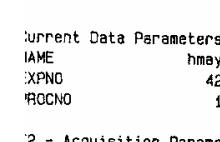

$2-$ Acquisition Paraneters
late- 20030519

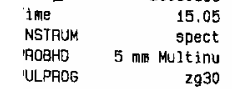

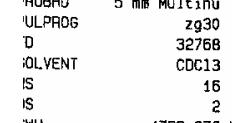

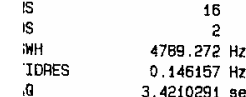

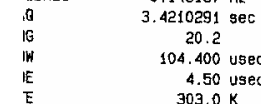

$\begin{array}{cc}\text { IE } & 4.50 \text { usec } \\ \text { E } & 3030 \mathrm{~K} \\ 11 & 1.00000000 \mathrm{~s} 80\end{array}$

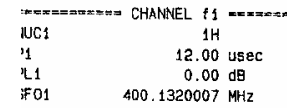

i. - Process ing parameters

$\begin{array}{ll}\text { iI } & \text { if } \\ \text { if } & 400.1300008 \mathrm{MHz} \\ \text { iD } & \mathrm{EM}\end{array}$

$\begin{array}{lc}\mathrm{NON} & \mathrm{EM} \\ \mathrm{iSB} & 0 \\ 3 \mathrm{~B} & 0.10 \mathrm{~Hz} \\ ; 8 & 0 \\ \mathrm{~B} & 1.00\end{array}$

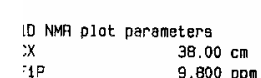

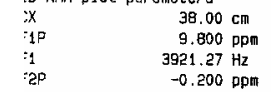

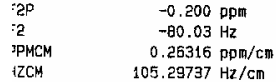

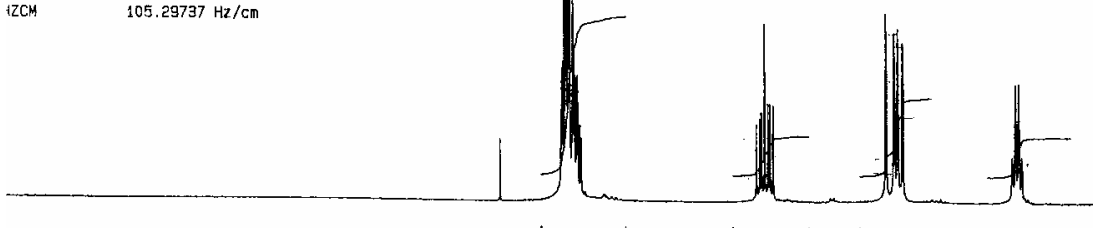

1

DPM

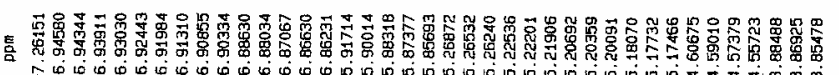

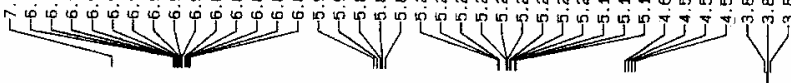

(12)

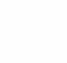

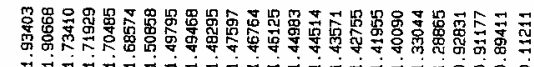

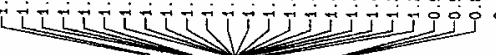

ता 
Current Data Parametors

$\begin{array}{lr}\text { NAME } & \text { CMay } \\ \text { EXPNO } & 35 \\ \text { PHOCNO } & 1\end{array}$

F2 - Acquisition Parameters

$\begin{array}{lr}\text { Date } & 20030519 \\ \text { Time } & 15.07\end{array}$

spect
INSTRUM
P

PULPAOG

TD $\quad 65536$

$\begin{array}{lr}\text { SOLVENT } & \text { COC } 13 \\ \text { NS } & 256\end{array}$

SWH $24691.257 \mathrm{~Hz}$

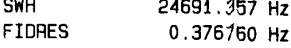

AQ $\quad 1.3271540 \mathrm{sec}$

$\begin{array}{ll}\text { AG } & 256 \\ \text { OW } & 20.250 \text { us }\end{array}$

$\begin{array}{lr}\text { DE } & 4.50 \text { use } \\ \text { TE } & 303.0 \mathrm{~K}\end{array}$

$0.03000000 \mathrm{sec}$

d12 $\quad 0.00002000 \mathrm{sec}$

$==2=== \pm==2$ CHANNEL $f 1$

$\begin{array}{ll}\text { NUC1 } & 13 \mathrm{C} \\ \text { P1 } & 10.00 \text { usec }\end{array}$

SF01 $100.6238523 \mathrm{MHZ}$

$z==x=z=3==$ CHANNEL $f 2$
CPDPAG

NUC2 $1 \mathrm{H}$

$\begin{array}{lr}\text { PL2 } & 88.00 \text { use } \\ \text { PLi2 } & 0.00 \mathrm{~dB}\end{array}$

PL13 13

SF02 400.1315005 MHz

F2 - Processing parameters

SI $\quad 32768$

SF $\quad 100.6127702 \mathrm{MHz}$

$\begin{array}{lc}\text { WDW } & E M \\ \text { SSB } & 0 \\ \text { LB } & 1.00 \mathrm{~Hz}\end{array}$

$G B$
$P C$

10 NMA piot parameters

CX $\quad 38.00 \mathrm{~cm}$

$38.00 \mathrm{~cm}$
F1P

$\begin{array}{lr}232.965 \mathrm{ppm} \\ F_{1} 1 & 23439.25 \mathrm{~Hz} \\ F_{2 p} & -12.650 \mathrm{ppm}\end{array}$

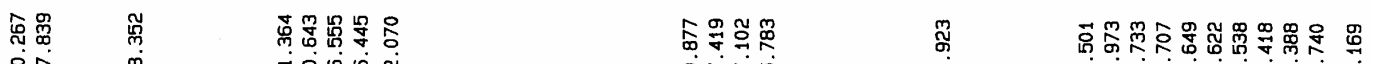

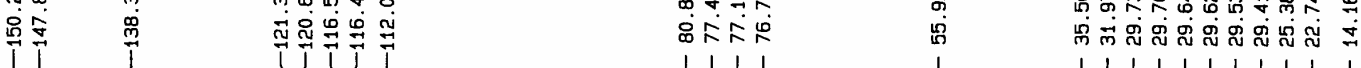

$-12.650 \mathrm{ppm}$
EPMCM

HZCM $\quad 5.46355 \mathrm{ppm} / \mathrm{cm}$ 
2-Methoxy-6-(pentadec-2-enyl)phenol 11c

AMJ 388
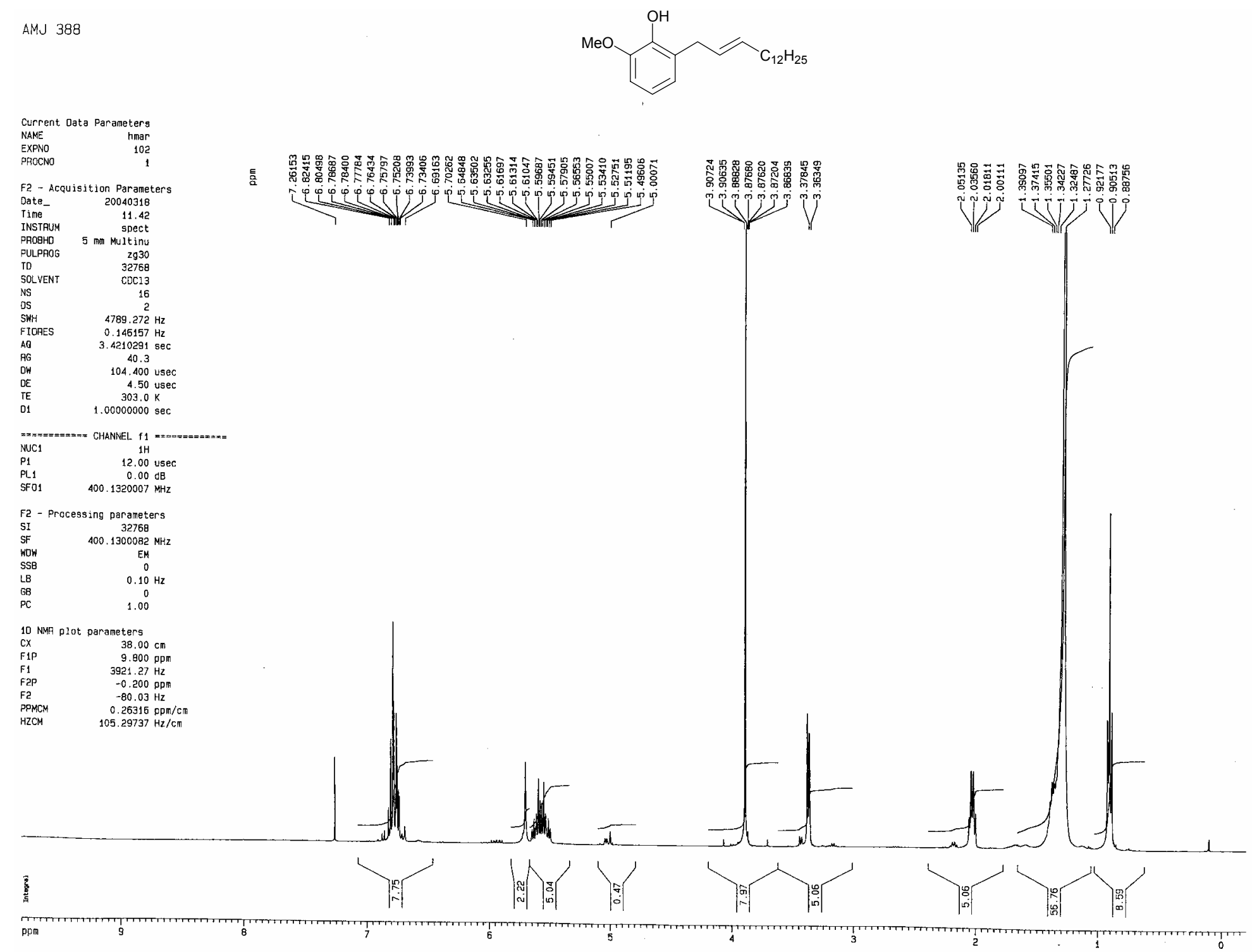
AMJ 388
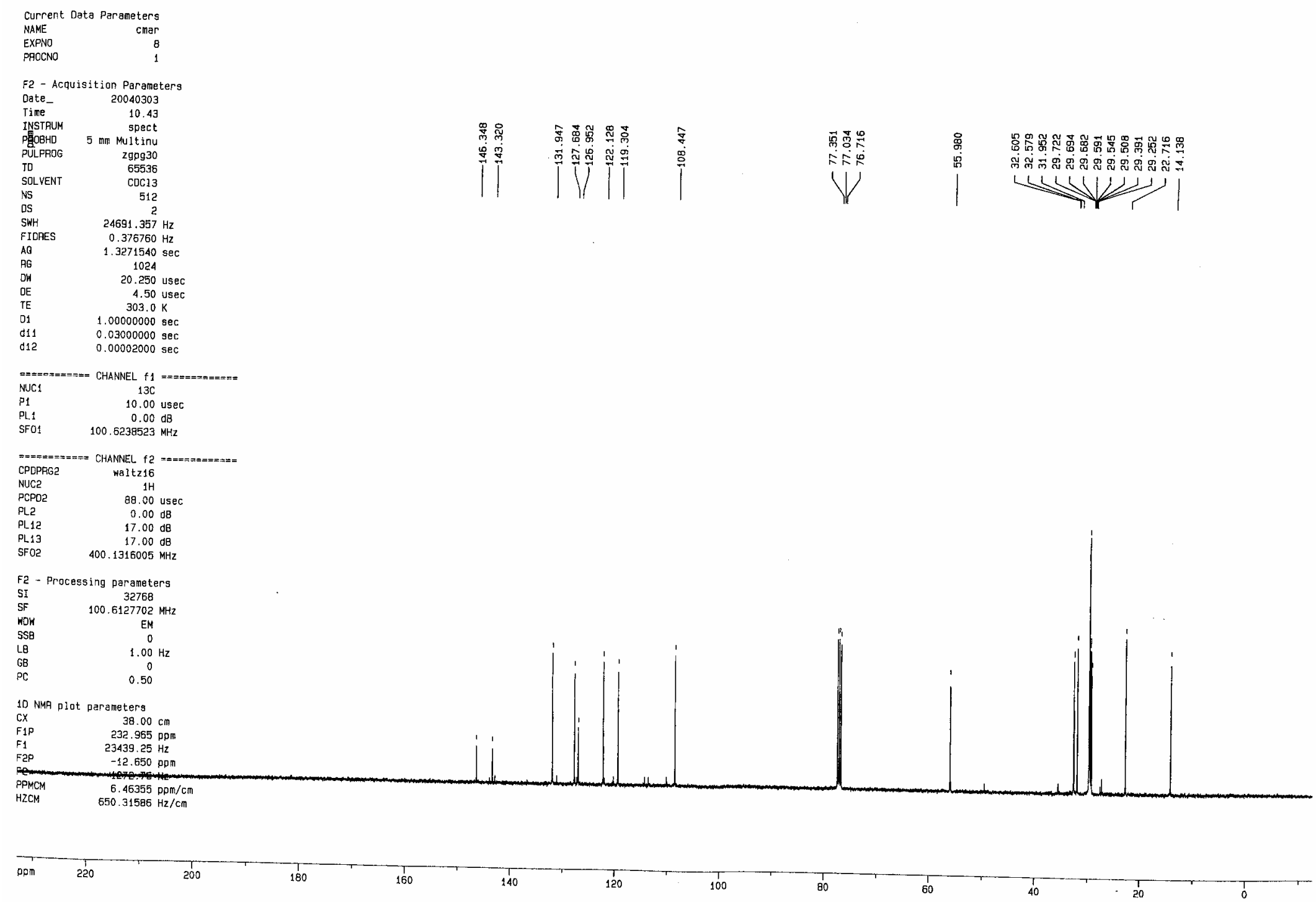
2-Methoxy-6-pentadecylphenol 12c

AMJ 201
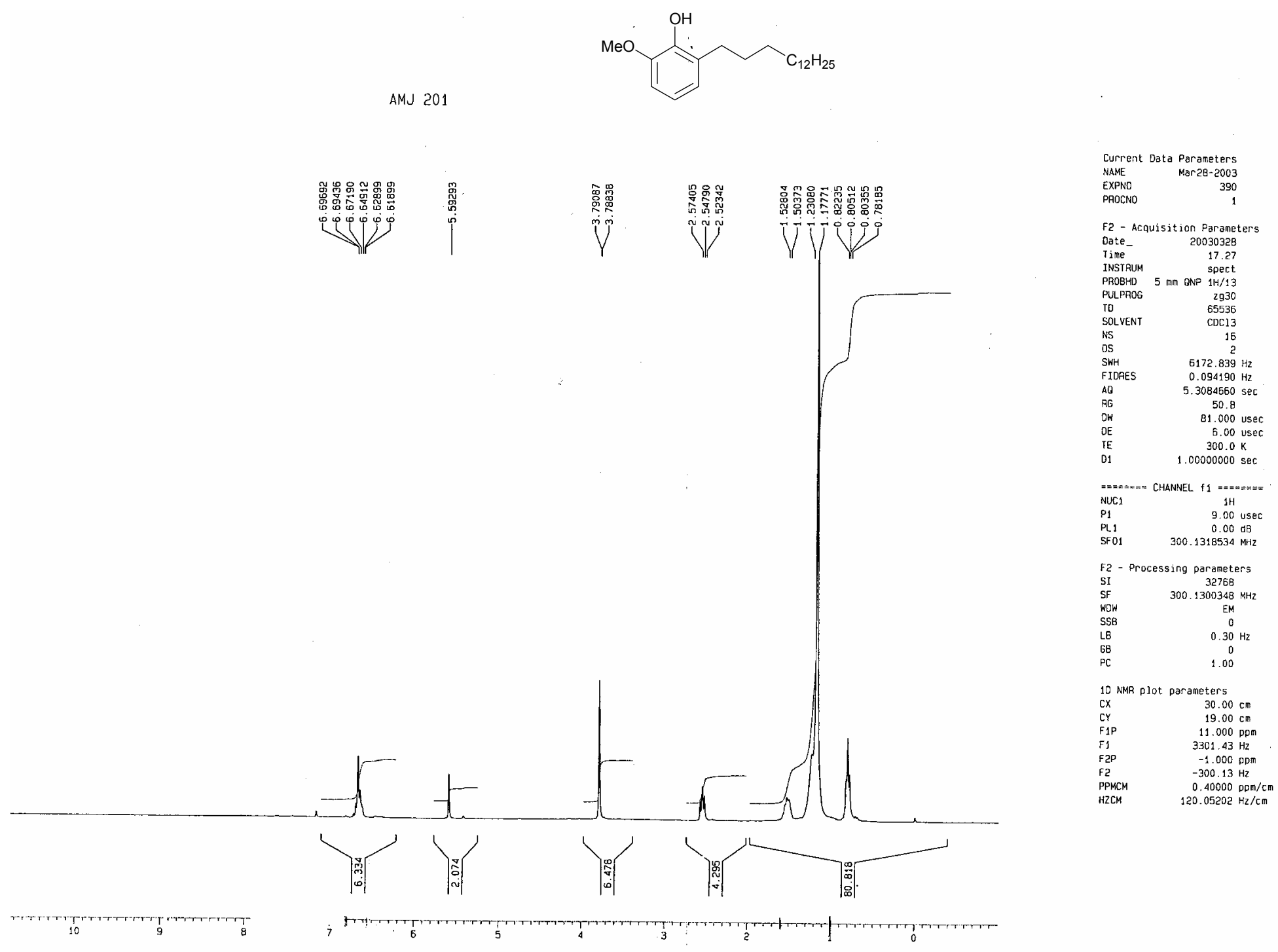
AMJ 201
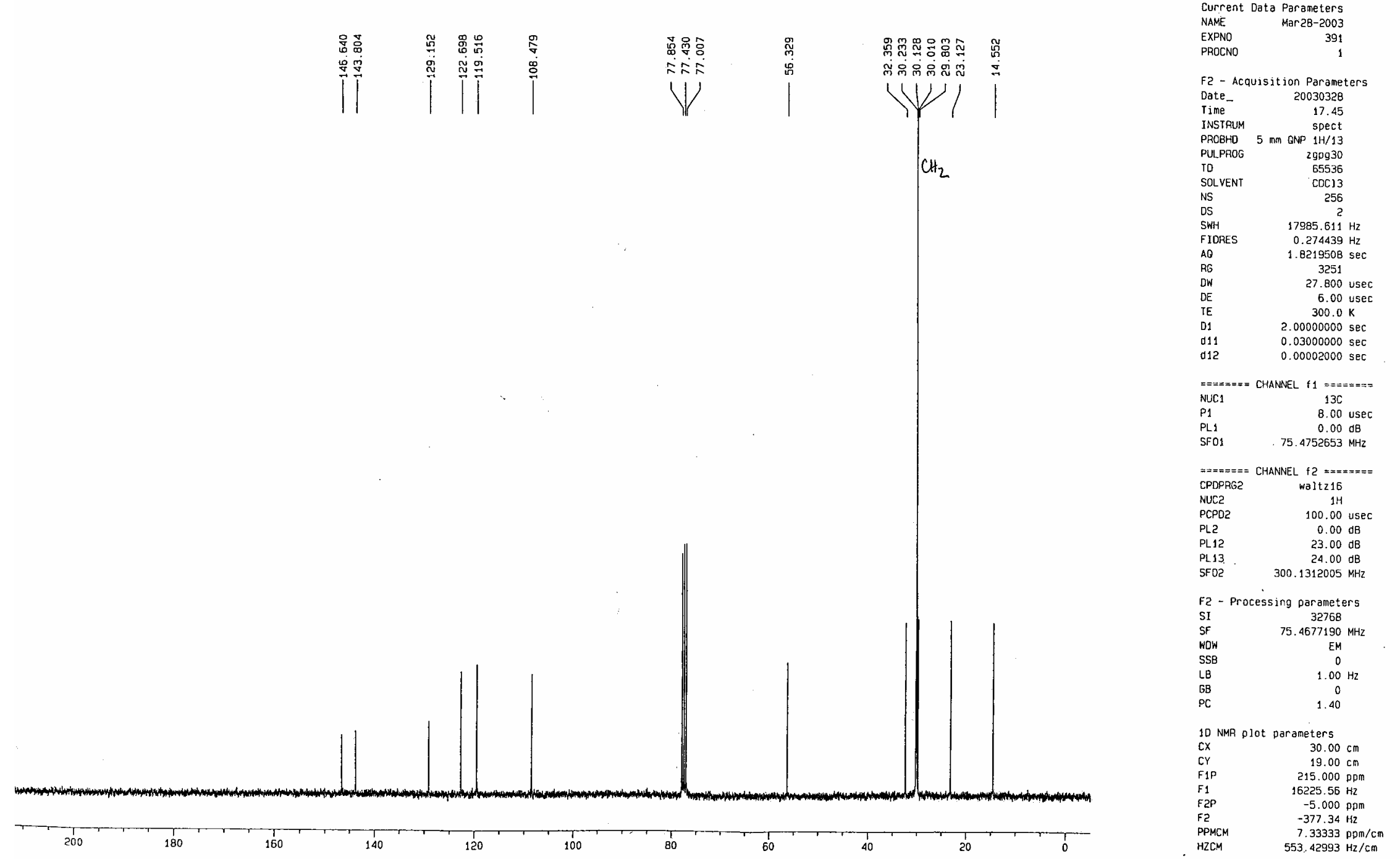
2-Methoxy-6-pentadecyl-1,4-benzoquinone 3

AMJ 188
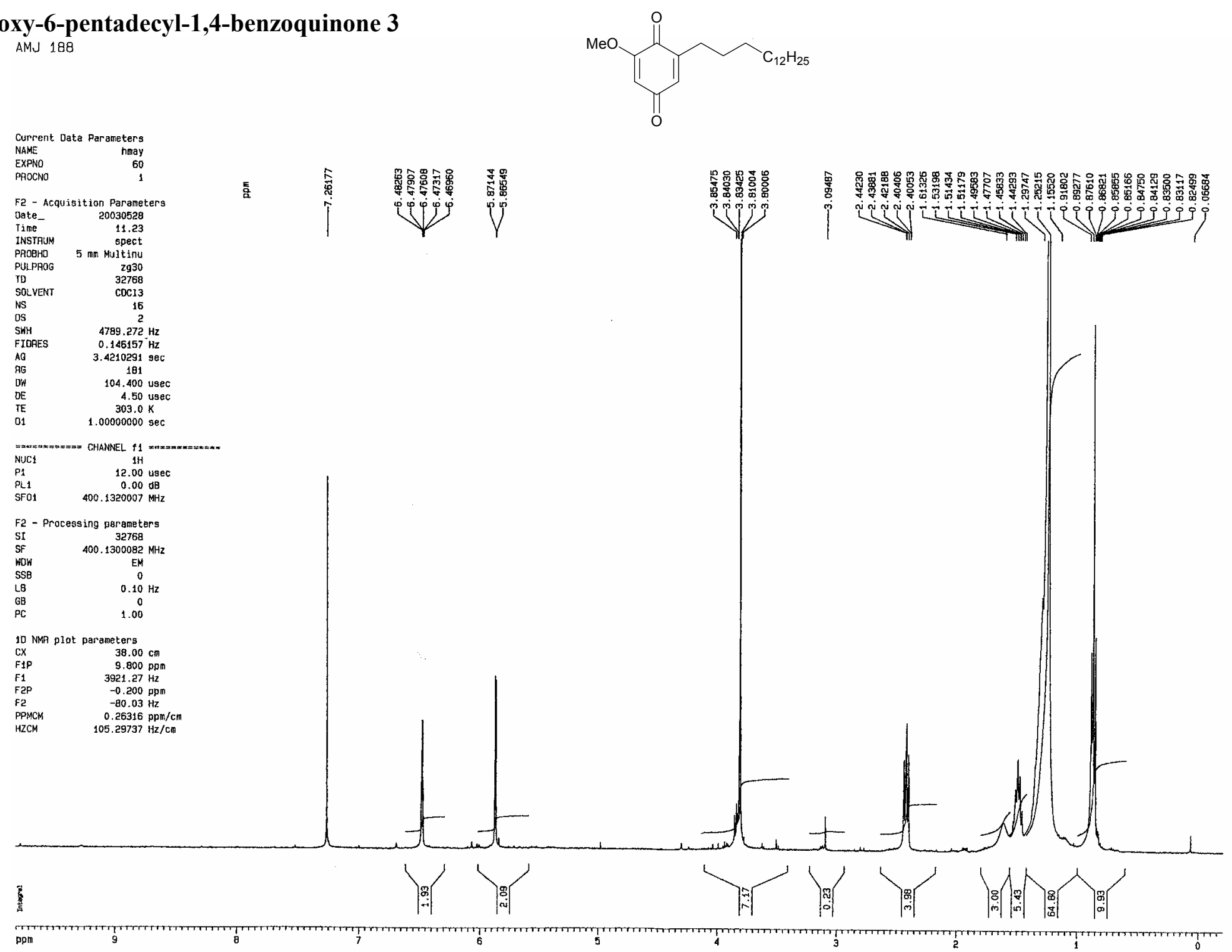
AMJ 188
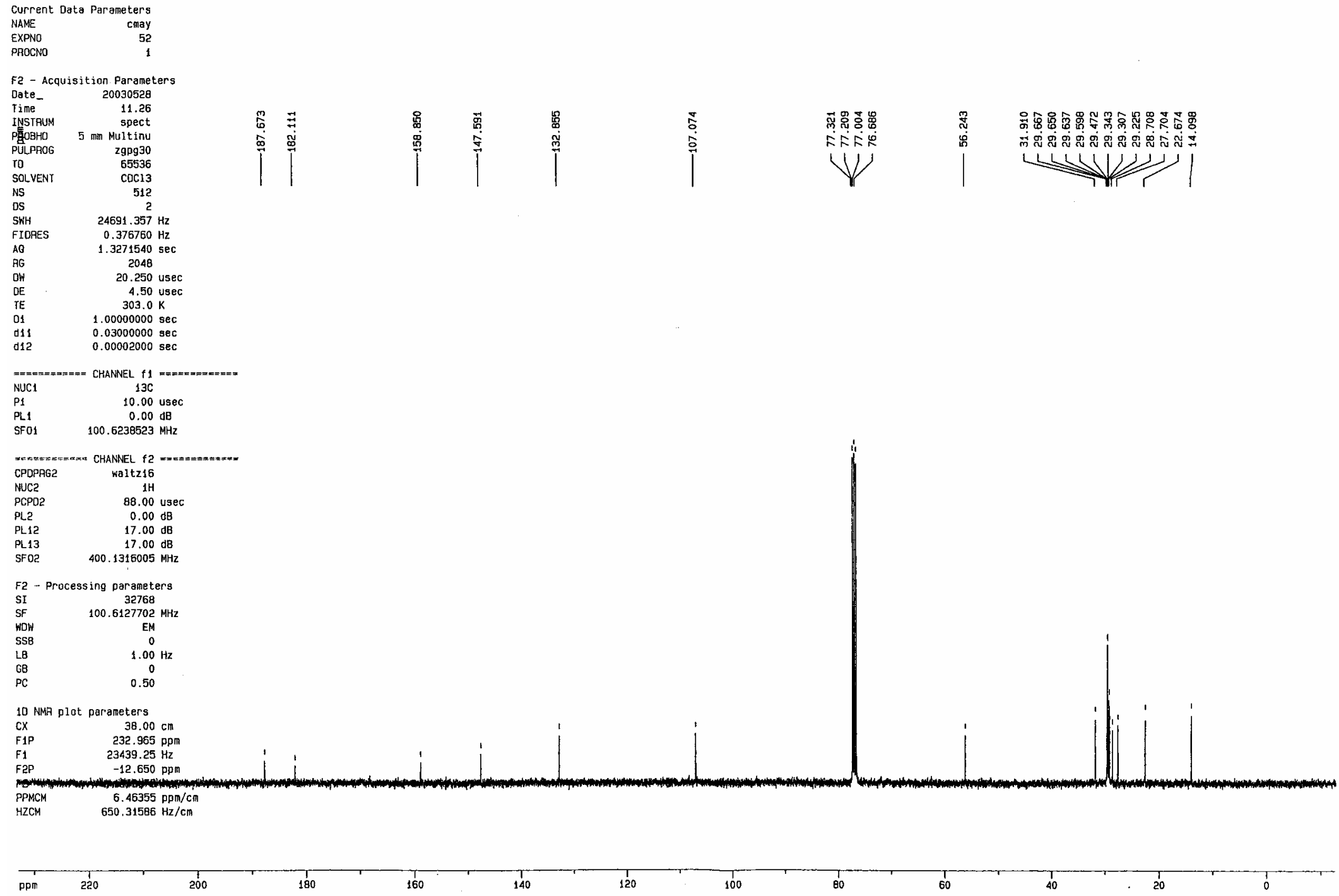
Heptadec-1-en-3-ol

$$
\overbrace{14}^{\mathrm{C}_{14} \mathrm{H}_{29}}
$$

cjo 482
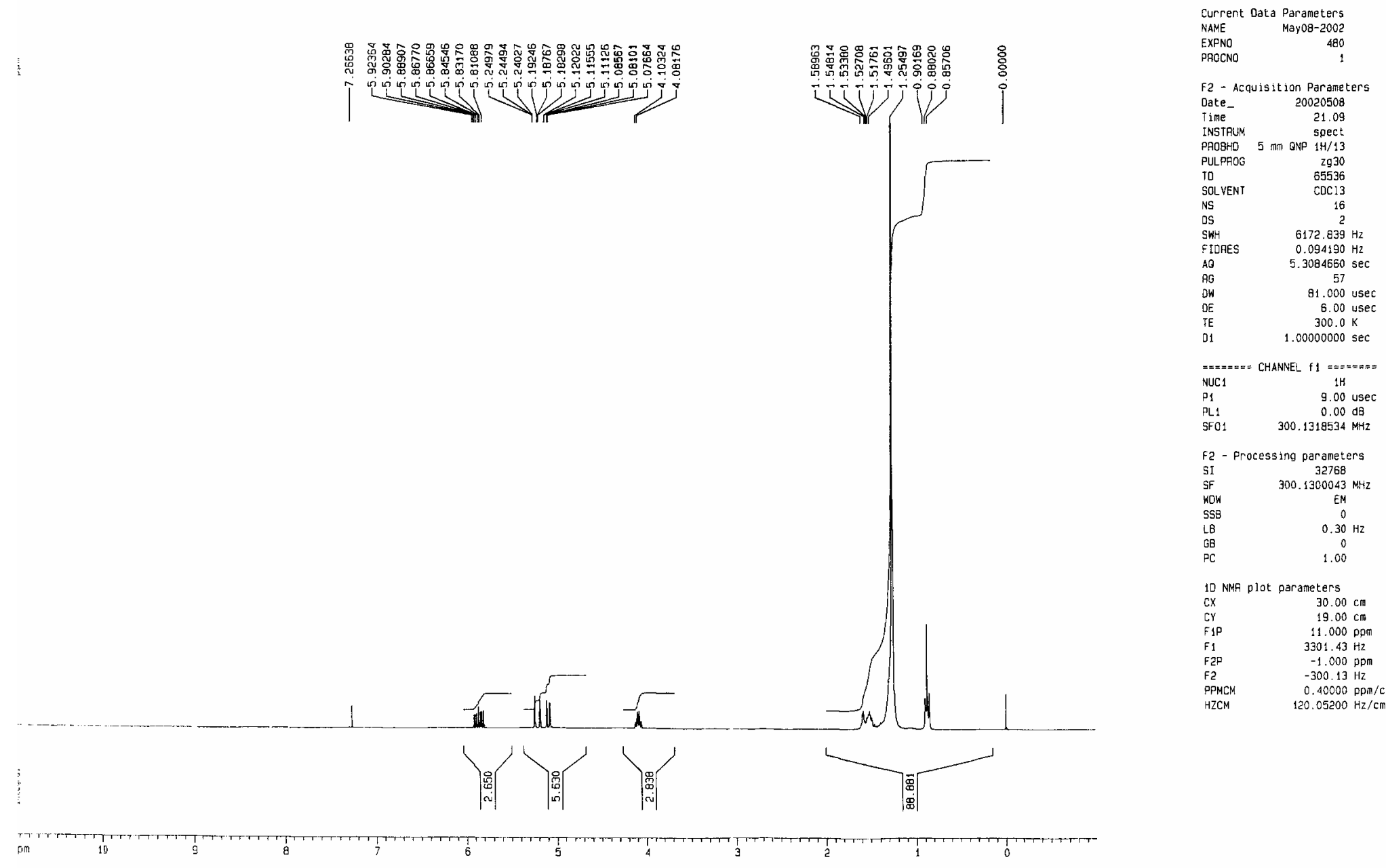
c)

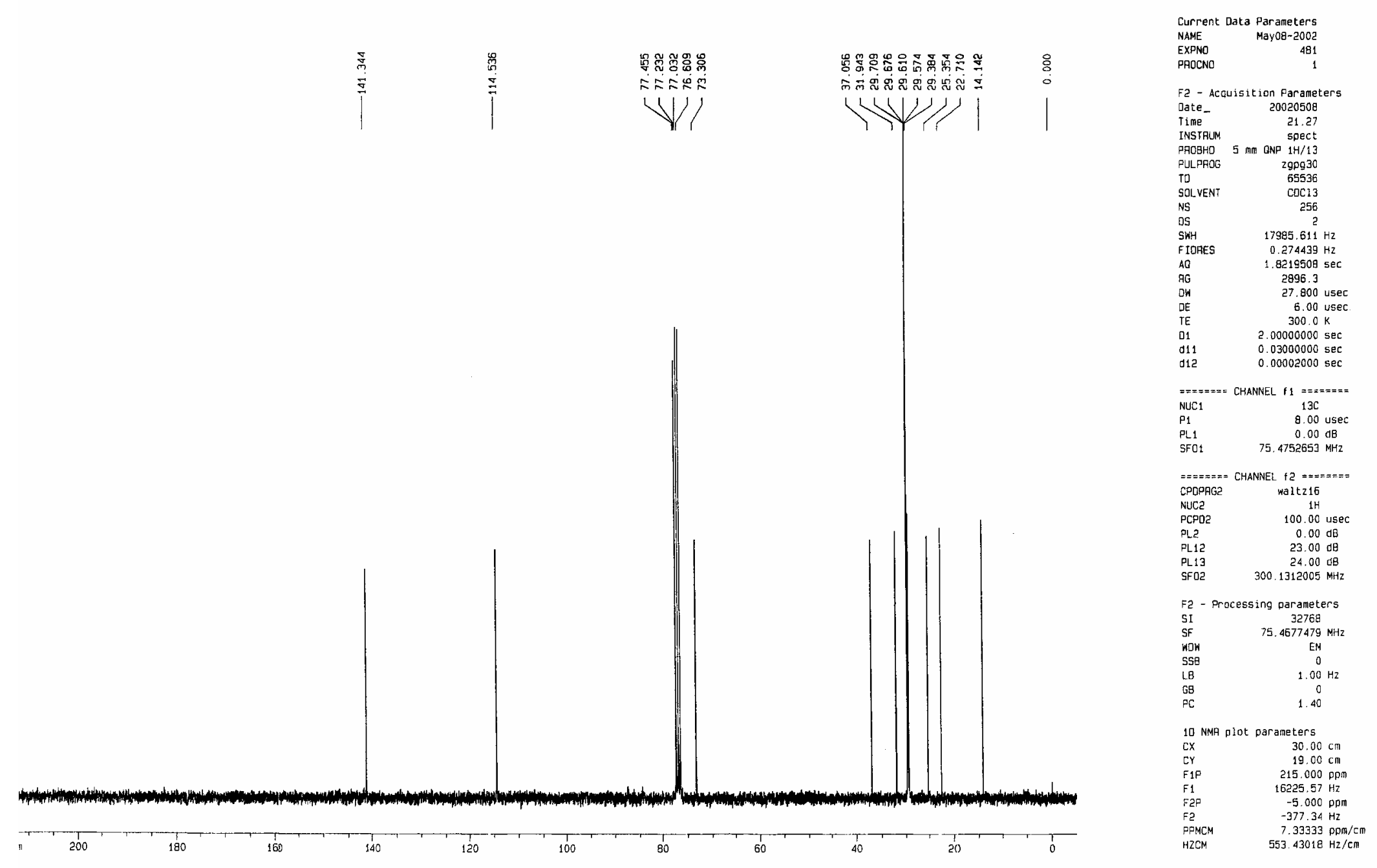


1-Methoxy-2-(heptadec-1-en-3-yloxy)benzene 10d<smiles>C=CC(CC)Oc1ccccc1OC</smiles>
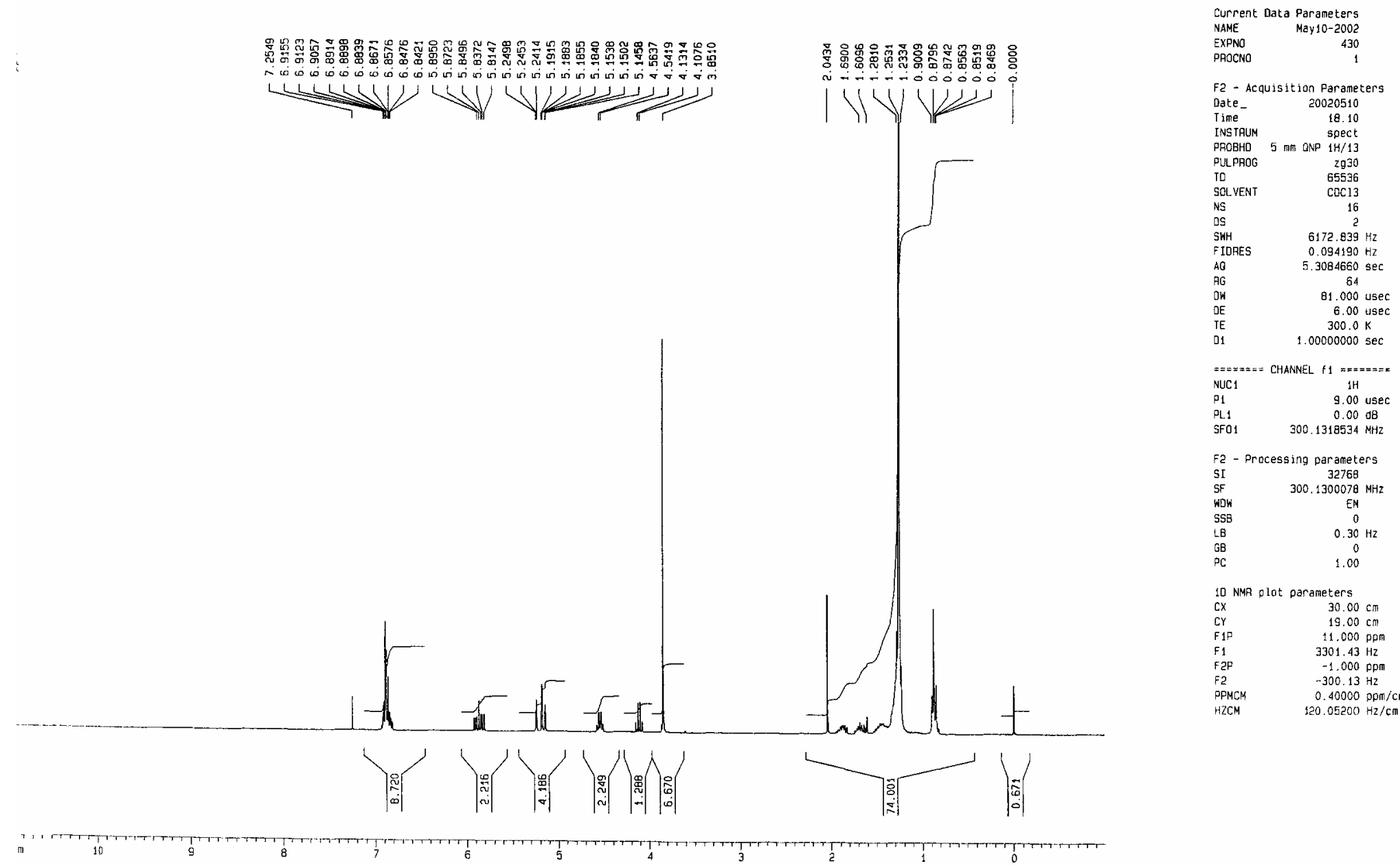
cj0485
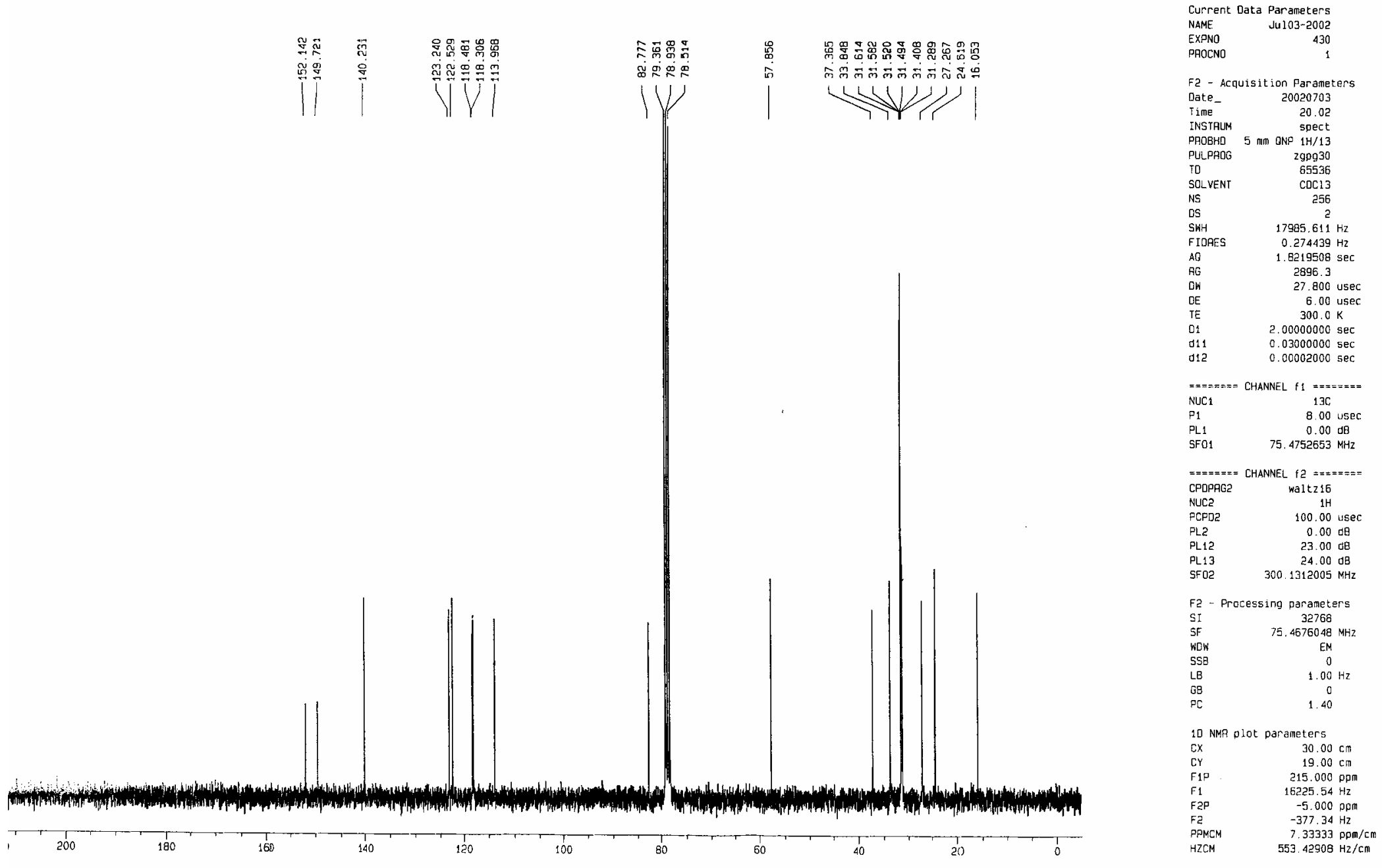
2-Heptadec-2-enyl-6-methoxyphenol 11d
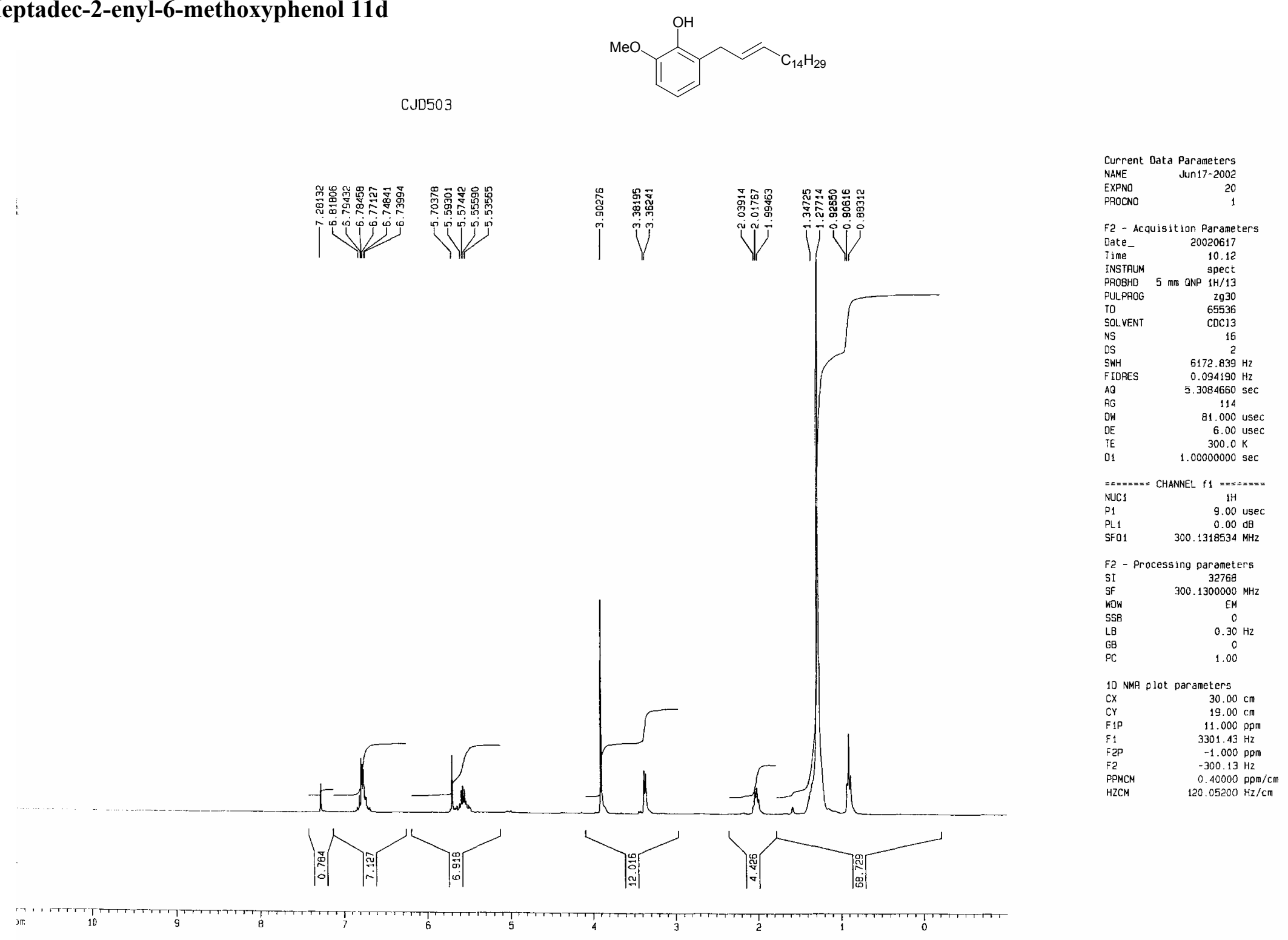
cj0503

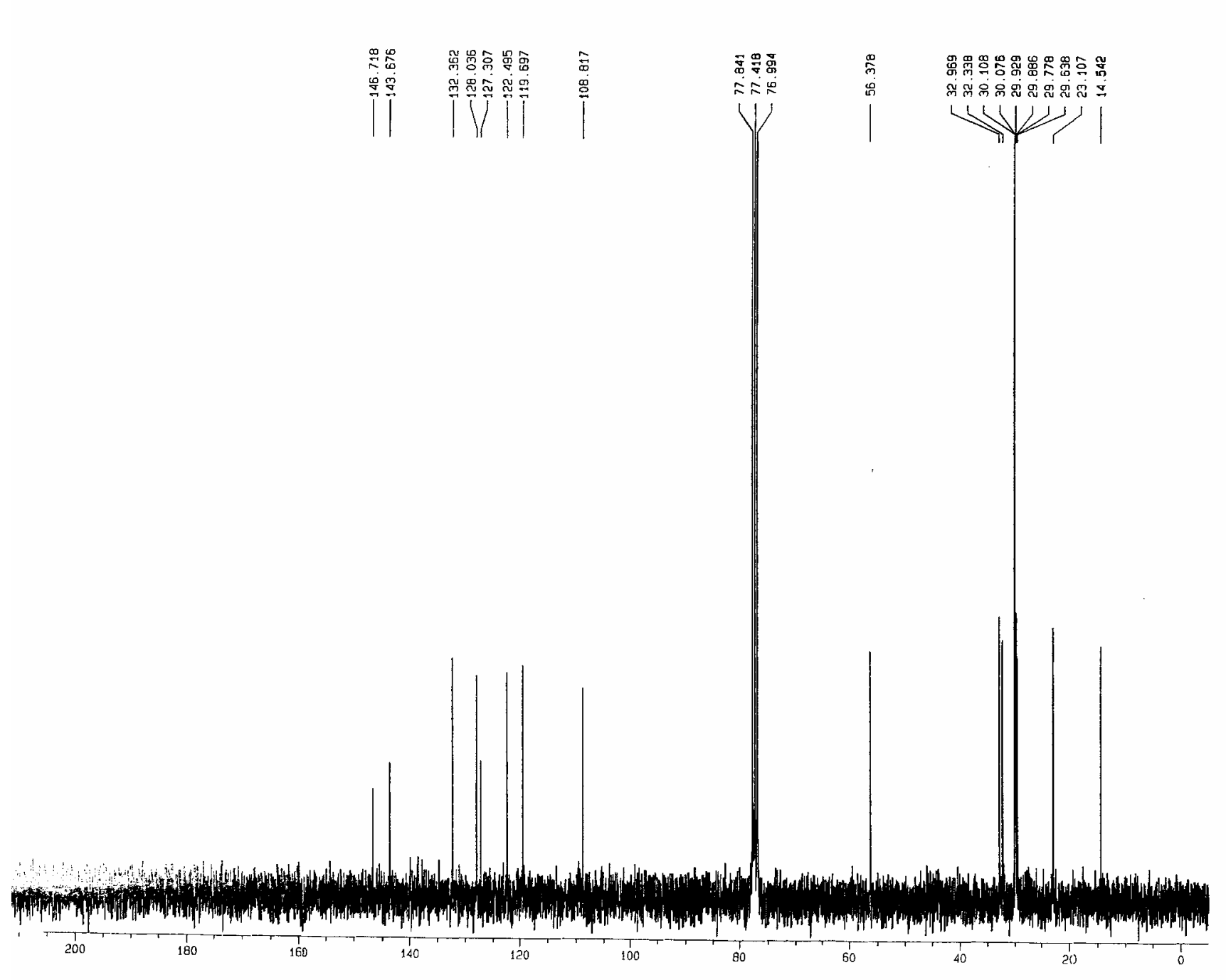

$$
\begin{aligned}
& \begin{array}{l}
\text { Current Data Parameters } \\
\text { NAME S Jun17-2002 } \\
\text { EXPNO }
\end{array} \\
& \text { PROCNO } \\
& \text { F2 - Acquisition Parameters } \\
& \text { Date } 20020617 \\
& \begin{array}{ll}
12.38 \\
\text { INSTFUM } \\
\text { PROBHD }
\end{array} \\
& \begin{array}{lr}
\text { PULPAOG } & \text { IDP } \\
\text { ID } & 65330 \\
\end{array} \\
& \begin{array}{lr}
\text { SOLVENT } & \text { COC13 } \\
\text { NS } & 256 \\
\text { OS } & 5
\end{array} \\
& \begin{array}{lr}
\text { SWH } & \multicolumn{2}{c}{} \\
\text { FHDFES } & 17985.611 \mathrm{~Hz} \\
\text { FIDES } & 0.274439 \mathrm{~Hz}
\end{array} \\
& \begin{array}{ll}
\text { AQ } & 0.274439 \mathrm{~Hz} \\
\text { AG } & 1.8219508 \mathrm{sec}
\end{array} \\
& \begin{array}{lr}
\text { RG } & 5792.6 \\
\text { OW } & 27.800 \text { usec } \\
\text { DE } & 6.00 \text { usec } \\
\text { TE } & 50000 \text {. }
\end{array} \\
& \begin{array}{ll}
01 & 300.0 \mathrm{~K} \\
01 & 00000000 \mathrm{sec}
\end{array} \\
& \begin{array}{ll}
\text { di1 } & 0.03000000 \mathrm{sec} \\
\mathrm{dt2} & 0.00002000 \mathrm{sec}
\end{array}
\end{aligned}
$$

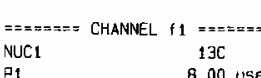

$$
\begin{aligned}
& \begin{array}{lr}
\text { NUC1 } & 13 C \\
\text { PL } & 8.00 \mathrm{usec} \\
\text { PL1 } & 0.00 \mathrm{oB} \\
\text { SFO1 } & 75.4752653 \mathrm{MHz}
\end{array} \\
& \begin{array}{l}
= \pm===z=\text { CHANNEL } f 2=z== \pm=- \\
\text { CPOPRGS }
\end{array} \\
& \begin{array}{lc}
\text { CPOPAG } & \text { Maltz16 } \\
\text { NUC2 } & 1 \mathrm{H} \\
\text { PCPO2 } & 100.00 \mathrm{usec} \\
\text { PL2 } & 0.00 \mathrm{~dB} \\
\text { PL2 } & 23.00 \mathrm{~dB}
\end{array} \\
& \begin{array}{lr}
\text { PL12 } & 23.00 \mathrm{~dB} \\
\text { PL13 } & 24.00 \mathrm{~dB} \\
\text { SF02 } & 300.1312005 \mathrm{MHZ}
\end{array} \\
& \begin{array}{lr}
\text { F2 - Processing parameters } \\
\text { SI } & 32768 \\
\text { SF } & 75.4677190 \\
\text { MHz }
\end{array} \\
& \begin{array}{ll}
\text { SF } & 75.4677190 \text { MHI } \\
\text { WWW } & \text { EM }
\end{array} \\
& \begin{array}{lc}
\text { SSB } & 0 \\
\text { LB } & 1.00 \mathrm{~Hz} \\
\text { GB } & 0
\end{array} \\
& \begin{array}{l}
\text { 10 NMP plot parameters } \\
\text { CX } \\
30.00 \mathrm{c}
\end{array} \\
& \begin{array}{lr}
C X & 30.00 \mathrm{~cm} \\
C Y & 19.00 \mathrm{~cm} \\
F_{1 P} & 215.000 \mathrm{ppm} \\
\mathrm{F}_{1} & 1525.55 \mathrm{mz}
\end{array} \\
& \begin{array}{ll}
F 1 & 16225.56 \mathrm{~Hz} \\
\text { FeP } & -5.000
\end{array} \\
& \begin{array}{ll}
\text { F2 } & -377.34 \mathrm{~Hz} \\
\text { PPMCM } & 7.3339 \mathrm{PPR} / \mathrm{CH}
\end{array}
\end{aligned}
$$




\section{2-Heptadecyl-6-methoxyphenol 12d}

c j 4508
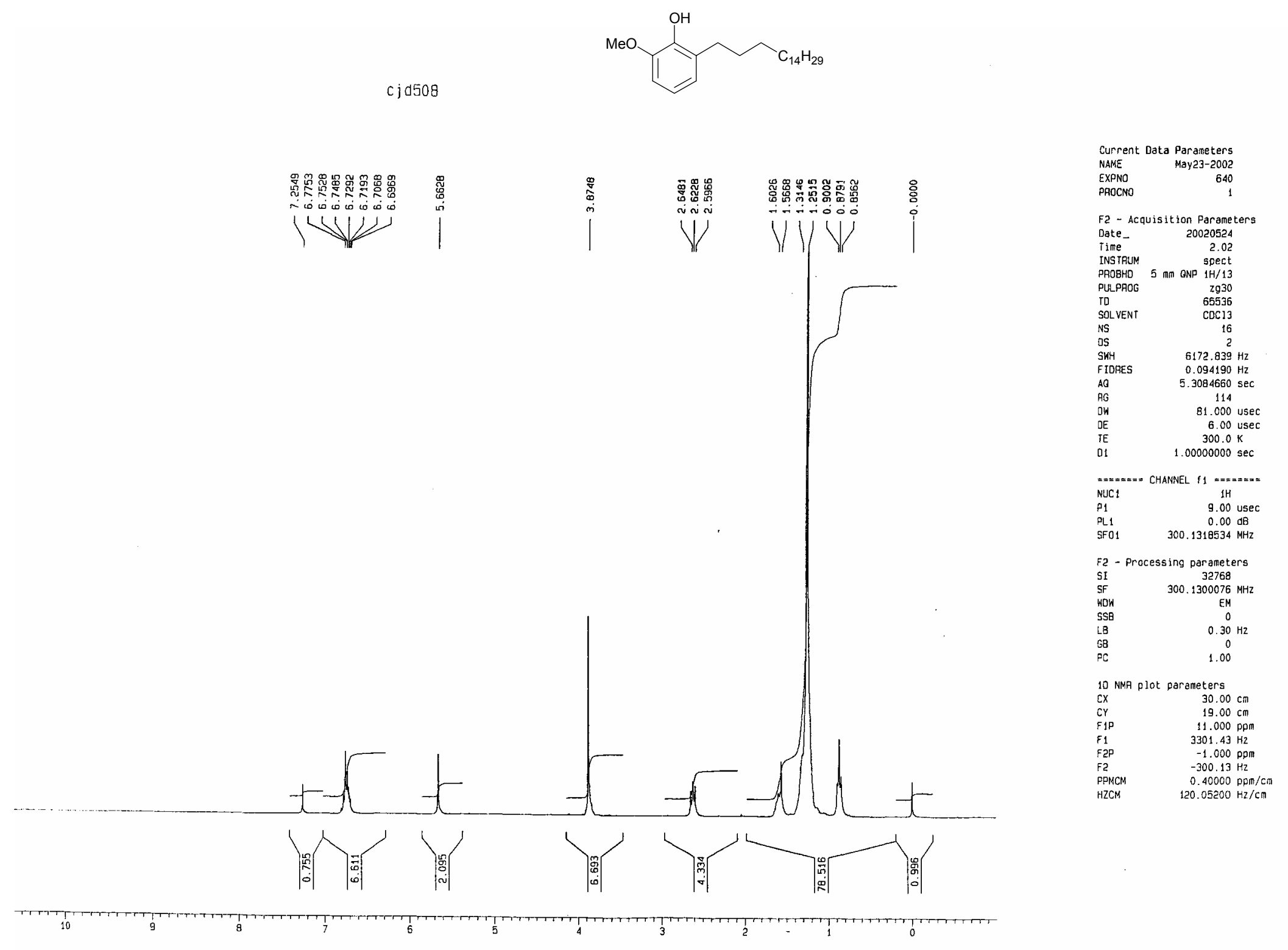


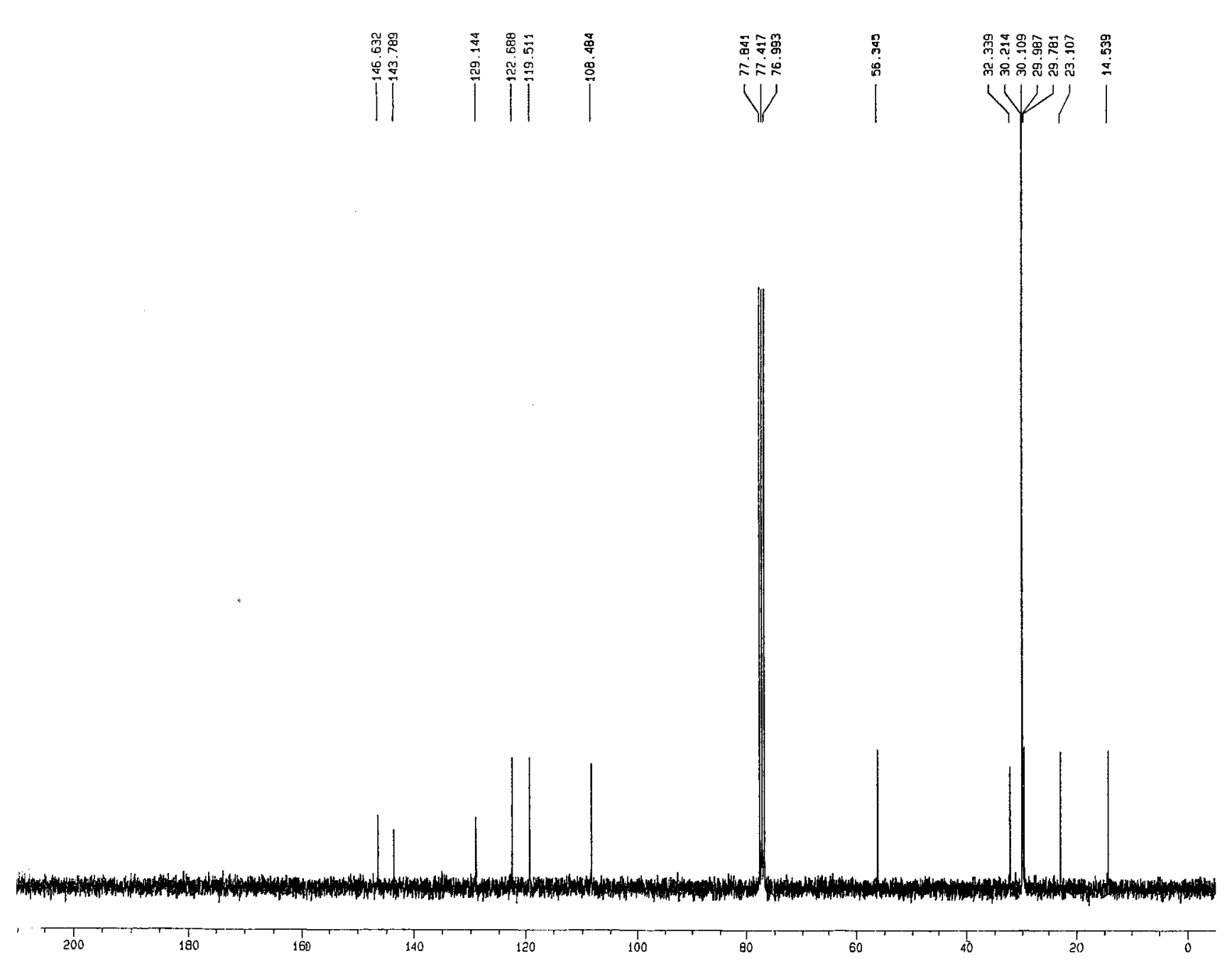

Current Data Parameters
NAME Mayz-200a
EXPND

EXPNO
PROCNO

F2 - Acquisition Parameters

$\begin{array}{ll}\text { Date_ } & 20020527 \\ \text { Time- } & 15.10\end{array}$

INSTRUM

PसOBHD 5 mm ONP $14 / 13$

$29 p 930$
ro

$\begin{array}{lr}\text { SOLVENT } & \text { CDC13 } \\ \text { NS } & 256 \\ \text { DS } & 256\end{array}$

$\begin{array}{lr}\text { SWH } & 17985.611 \mathrm{~Hz} \\ \text { FIDAES } & 0.274439 \mathrm{~Hz}\end{array}$

Aa $\quad 1.8219500 \mathrm{sec}$

$\begin{array}{lr}\text { AG } & 3251 \\ \text { DW } & 27.800 \mathrm{usec} \\ \text { OE } & 6.00 \mathrm{usec}\end{array}$

JE $\quad 300.0 \mathrm{~K}$

d11 $\quad 0.03000000 \mathrm{sec}$

d12 $\quad 0.00002000 \mathrm{sec}$

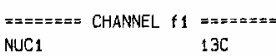

$\begin{array}{ll}\text { P1 } & 13 C \\ \text { PL1 } & 8.00 \text { USE }\end{array}$

$\begin{array}{lr}\text { PL1 } & 0.00 \mathrm{~dB}\end{array}$

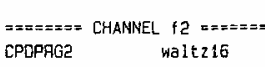

$\begin{array}{ll}\text { NuC2 } & 1 \mathrm{H} \\ \text { PCPOE } & 100.00\end{array}$

$\begin{array}{lr}\text { PCPO2 } & 100.00 \text { use } \\ \text { PL.2 } & 0.00 \mathrm{dg} \\ \text { PL12 } & 23.000 \mathrm{~dB}\end{array}$

$24.00 \mathrm{~dB}$
$\mathrm{SFCO}$

$\begin{array}{lr}\text { F2 - Processing parameters } \\ \text { SI } & 32776 \\ \text { SE } & 75.4677190\end{array}$

$\begin{array}{lc}\text { SF } & 75.4677190 \mathrm{NH} \\ \text { WDW } & \text { EM } \\ \text { SSB } & 0\end{array}$

$\begin{array}{cc}\text { SSB } & 0 \\ \text { L } & 1.00 \mathrm{~Hz} \\ \text { G } & 0\end{array}$

10. NMF plot parameters

$\begin{array}{ll}\mathrm{CX} & 30.00 \mathrm{cll} \\ \mathrm{CY} & 19.00 \mathrm{~cm}\end{array}$

$\begin{array}{lr}\text { CY } & 19.00 \mathrm{~cm} \\ F_{11} & 215.000 \mathrm{ppm} \\ F_{1} & 16225.56 \mathrm{~Hz}\end{array}$

$\begin{array}{ll}\mathrm{F}_{2} & 1625.56 \mathrm{~Hz} \\ \mathrm{~F} & -5.000 \mathrm{ppm} \\ \mathrm{FP} & -377.34 \mathrm{~Hz}\end{array}$

$\begin{array}{lr}\mathrm{FP} & -377.34 \mathrm{~Hz} \\ \text { PPMCM } & 7.33333 \mathrm{pom} / \mathrm{cm} \\ \text { HZCM } & 553.42993 \mathrm{~Hz} / \mathrm{cm}\end{array}$ 
2-Heptadecyl-6-methoxy-1,4-benzoquinone (pallasone B) 4
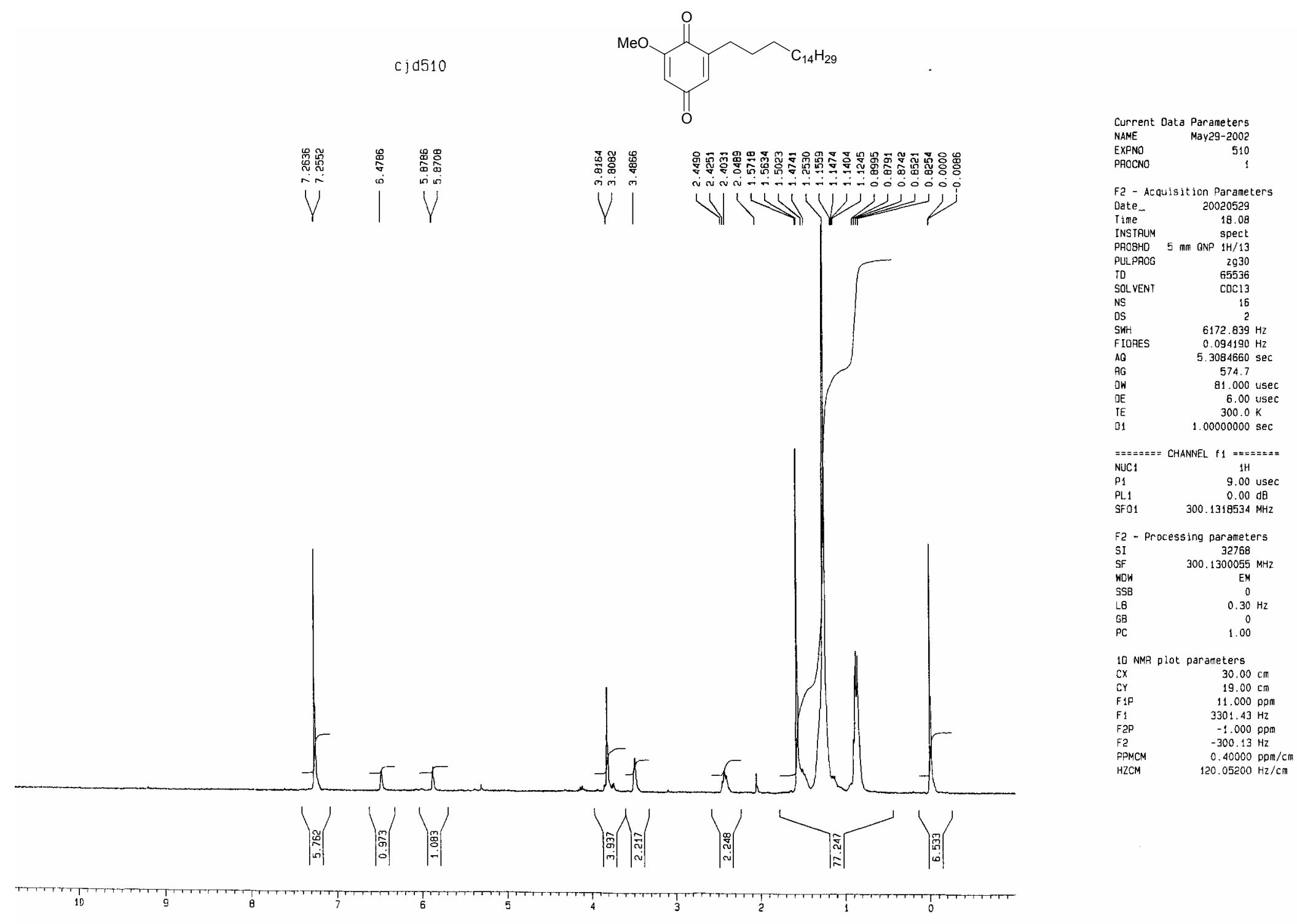
CJ0510

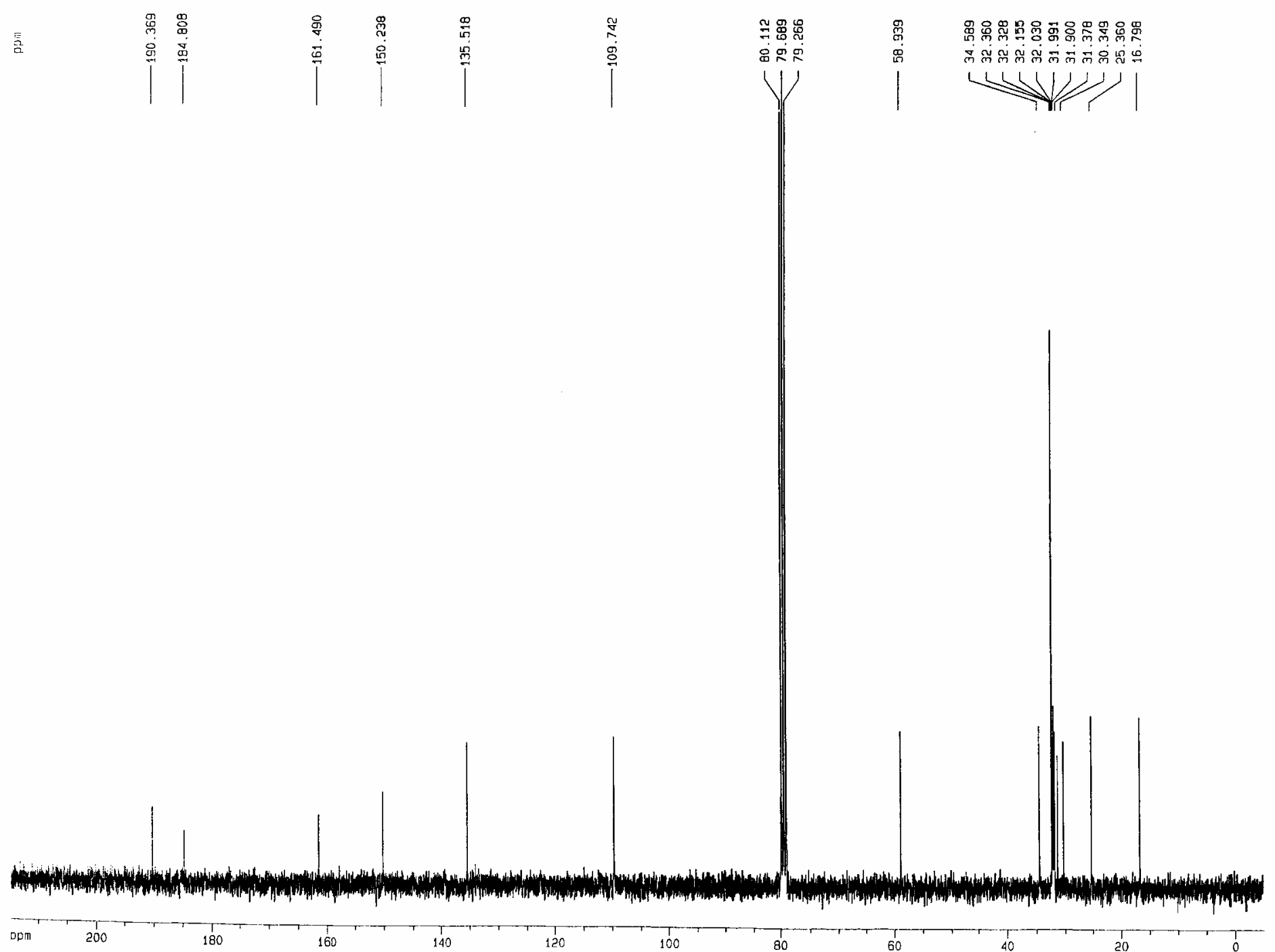

Current Data Paraneters

NAME Jun07-2002

EXPND

1

Fa - Acquisition Parameters

$\begin{array}{ll}\text { Date_- } & 20020607 \\ \text { Time } & 15.55\end{array}$

INSTR

PAOBHO $5 \mathrm{mIM}$ ONP $\begin{array}{r}\text { spect } \\ 1 H / 13\end{array}$

PULPAOG 2 290930

$\begin{array}{lr}\text { TO } & 65536 \\ \text { SOLYENT } & \text { CDC13 } \\ \text { NS } & 256\end{array}$

SHH $17985.611 \mathrm{~Hz}$

AG $\quad 0.274439 \mathrm{~Hz}$

AG
AG
AG

$\begin{array}{lr}\text { OW } & 27.800 \text { usec } \\ \text { OE } & 5.00 \text { usec }\end{array}$

TE $300.0 \mathrm{~K}$

$\begin{array}{ll}01 & 2.00000000 \mathrm{sec} \\ d_{11} & 0.03000000 \mathrm{sec} \\ \mathrm{d} & 0.00002000 \mathrm{sec}\end{array}$

$=\approx *= \pm= \pm$ CHANNEL $f 1== \pm===$
NUC1 $13 \mathrm{C}$

$\begin{array}{lr}\text { P1 } & 8.00 \text { usec } \\ \text { PL1 } & 0.00 \mathrm{~dB} \\ \text { SFO1 } & 75.4752653 \mathrm{MHZ}\end{array}$

$== \pm=\equiv=$ CHANNEL $f 2==== \pm==$

CPDPAG
NUCR

$\begin{array}{lc}\text { NUC2 } & 1 \mathrm{H} \\ \text { PCPD2 } & 100.00 \text { usec }\end{array}$

$\begin{array}{lr}\text { PLL2 } & 0.00 \mathrm{~dB} \\ \text { PLI2 } & 23.00 \mathrm{~dB}\end{array}$

$24.00 \mathrm{~dB}$

$300.1312005 \mathrm{MH}$

F2- Processing peraneters

SF $\quad 75.4675478 \mathrm{MHZ}$

$\begin{array}{lc}\text { HOW } & E M \\ \text { SSE } & 0 \\ \text { LB } & 1.00 \mathrm{~Hz}\end{array}$

10 NMA plot parameters

$\begin{array}{ll}\text { CX } & 30.00 \mathrm{~cm} \\ \mathrm{CY} & 19.00 \mathrm{~cm}\end{array}$

$\begin{array}{ll}\text { F1P } & 215.000 \mathrm{pPm} \\ \text { F1 } & 16225.52 \mathrm{~Hz}\end{array}$

$\begin{array}{lr}F 1 & 1625.52 \mathrm{~Hz} \\ F 2 P & -5.000 \mathrm{pp} \\ F 2 & -377.33 \mathrm{~Hz}\end{array}$

$\begin{array}{ll}F 2 & -377.33 \mathrm{~Hz} \\ \text { PPMCM } & 7.33393 \mathrm{POm} / \mathrm{Cm}\end{array}$

HZCM $553.42865 \mathrm{~Hz} / \mathrm{cm}$ 


\section{3,7-Dimethyloct-6-en-1-yl-3-ol 13}

$\mathrm{MO}_{\mathrm{Me}}^{\mathrm{Me}}$

c) 0461
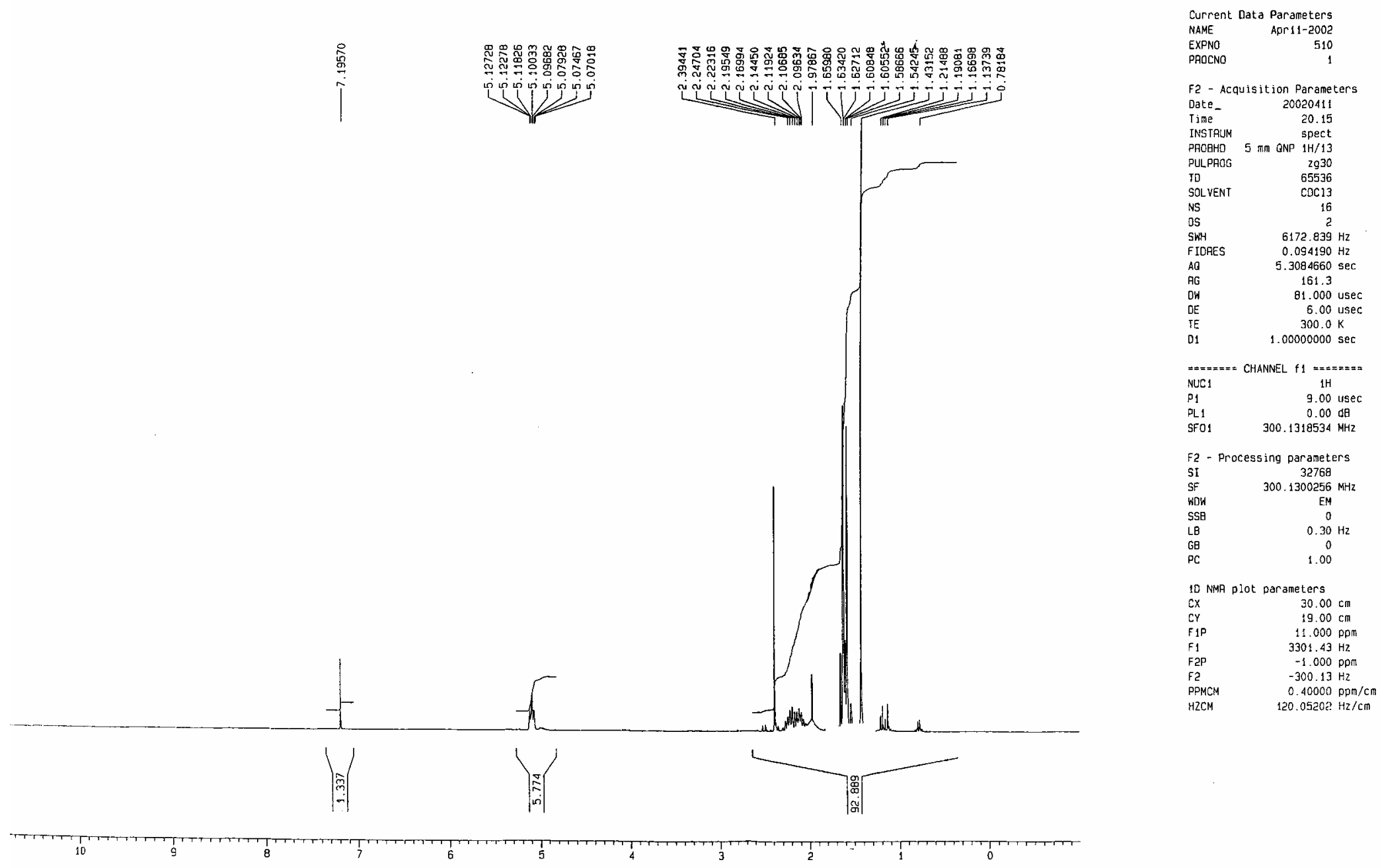

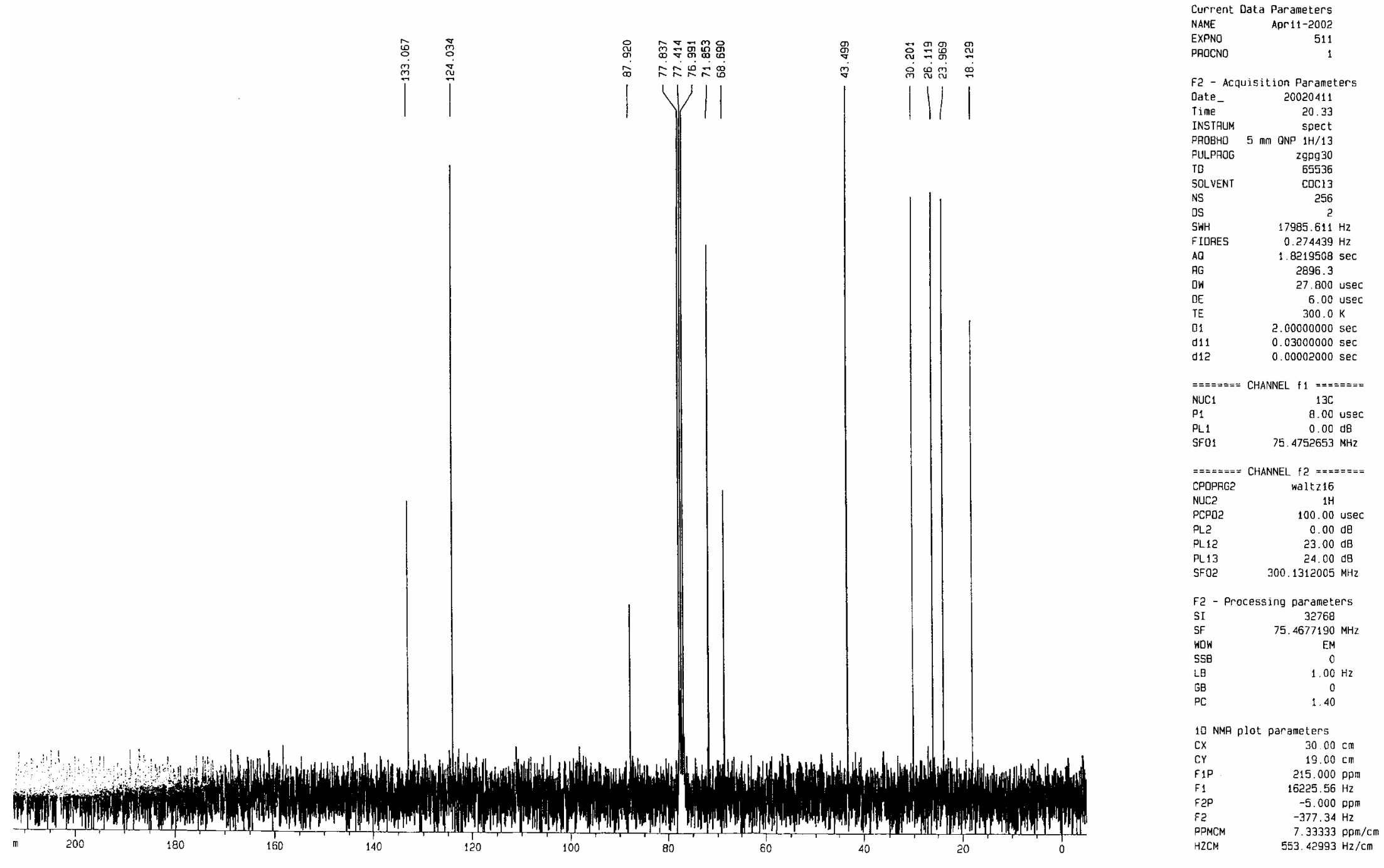
1-(3,7-Dimethyloct-6-en-1-yn-3-yloxy)-2-methoxybenzene 14

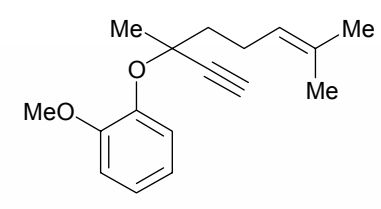

C.ปD492
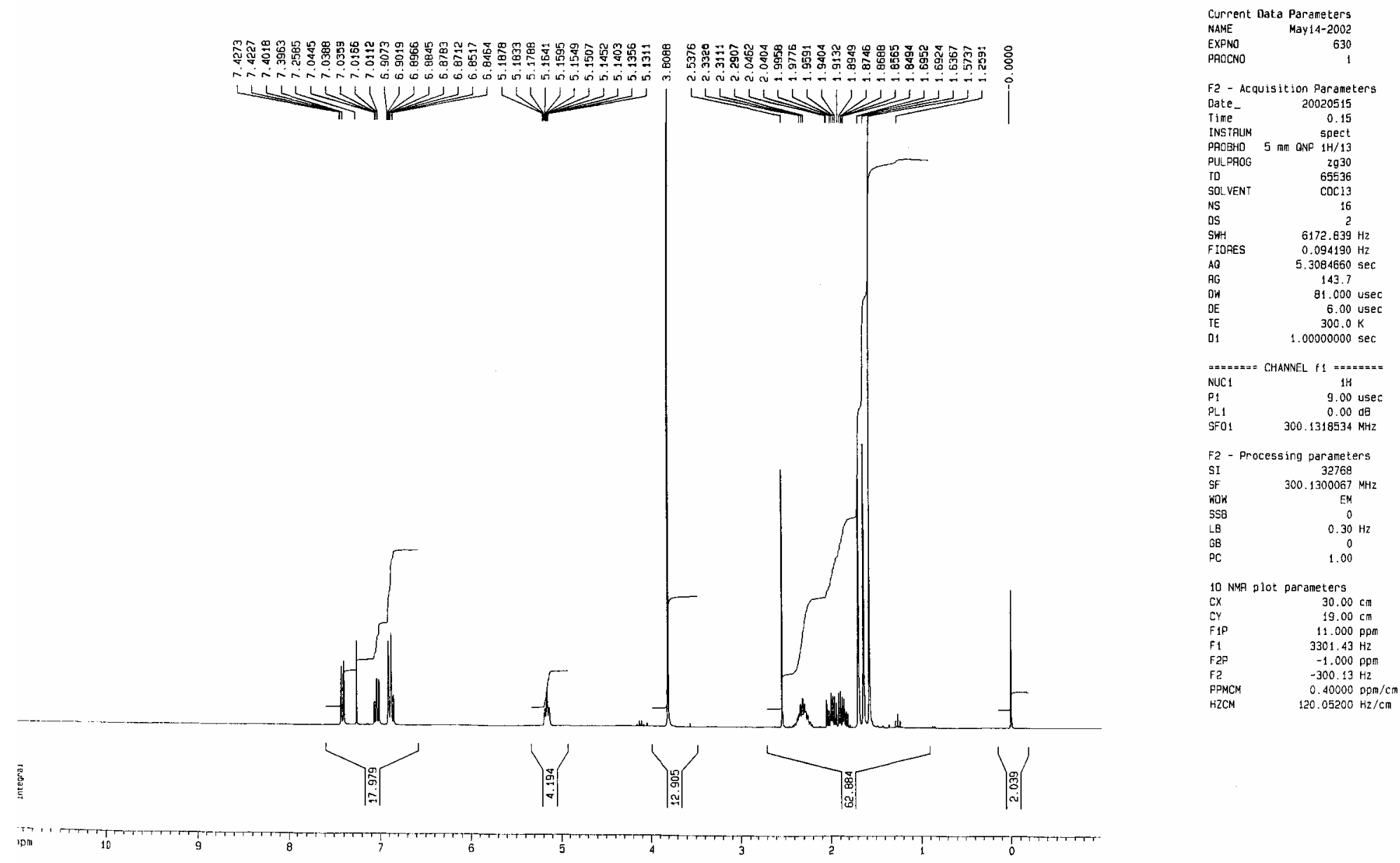


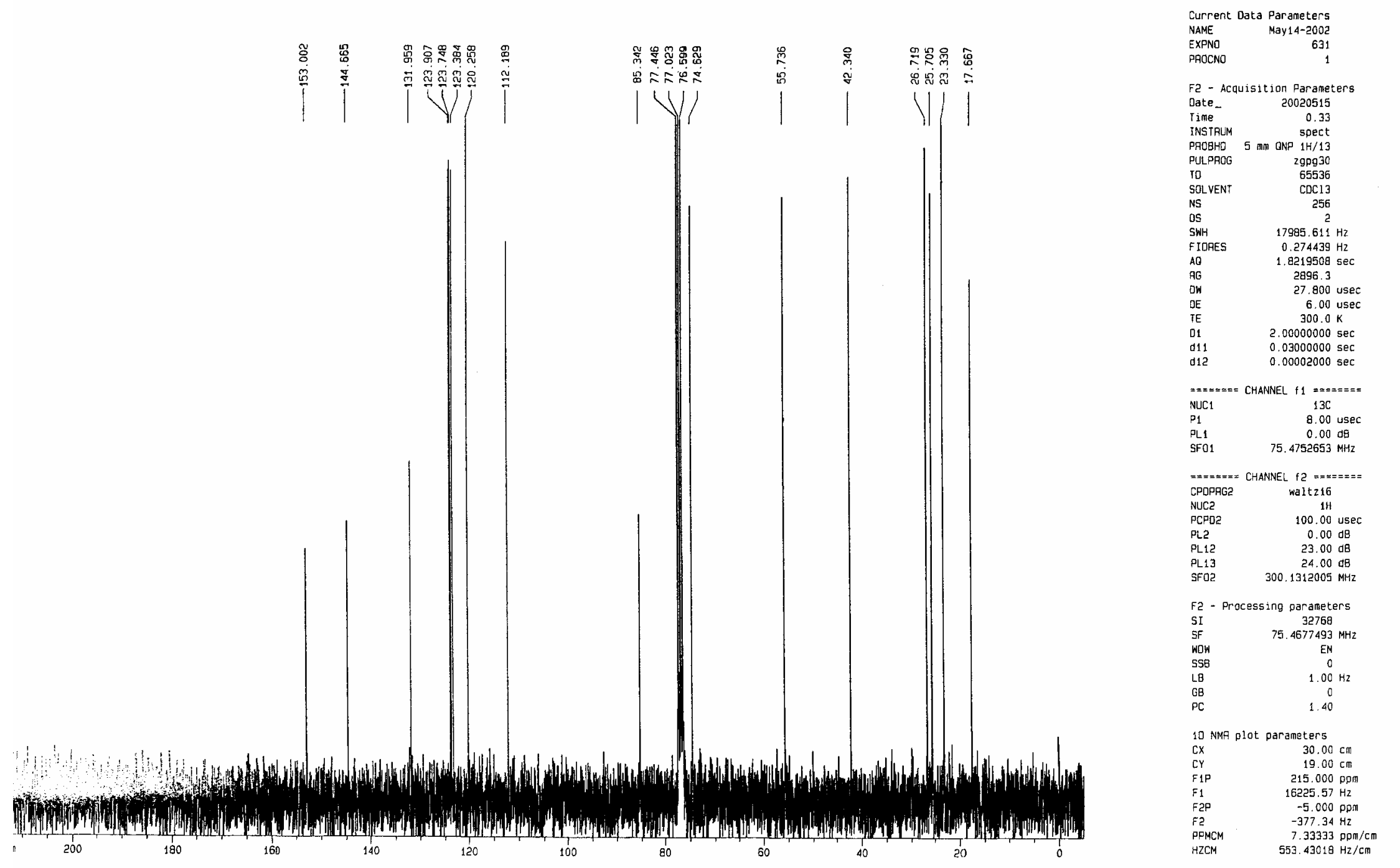


1-(3,7-Dimethylocta-1,6-dien-3-yloxy)-2-methoxybenzene 15

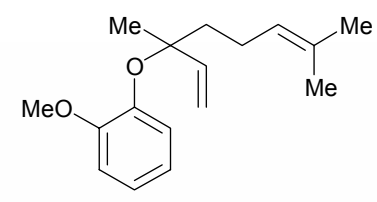

cjo497
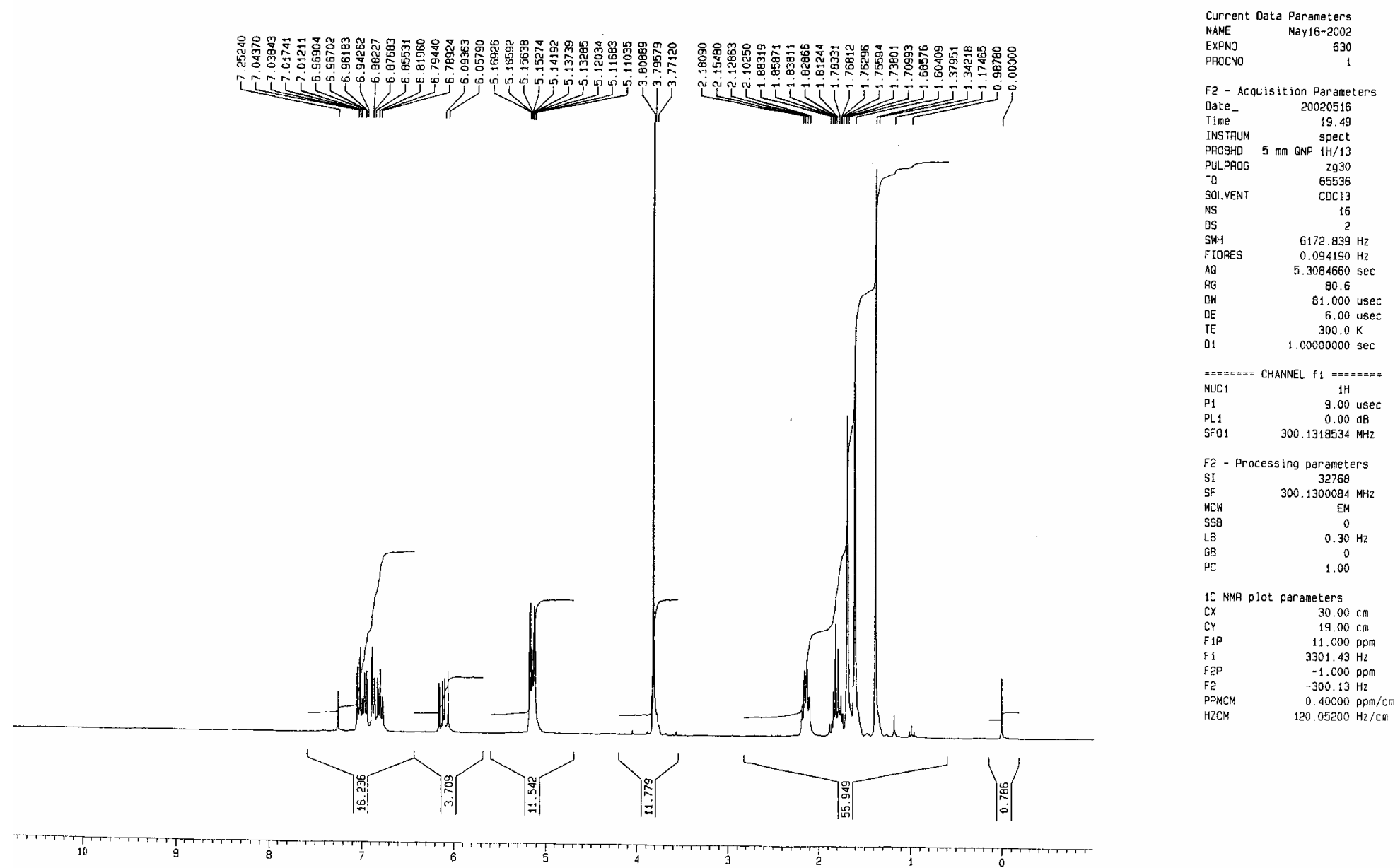

$\frac{1}{5}$ 

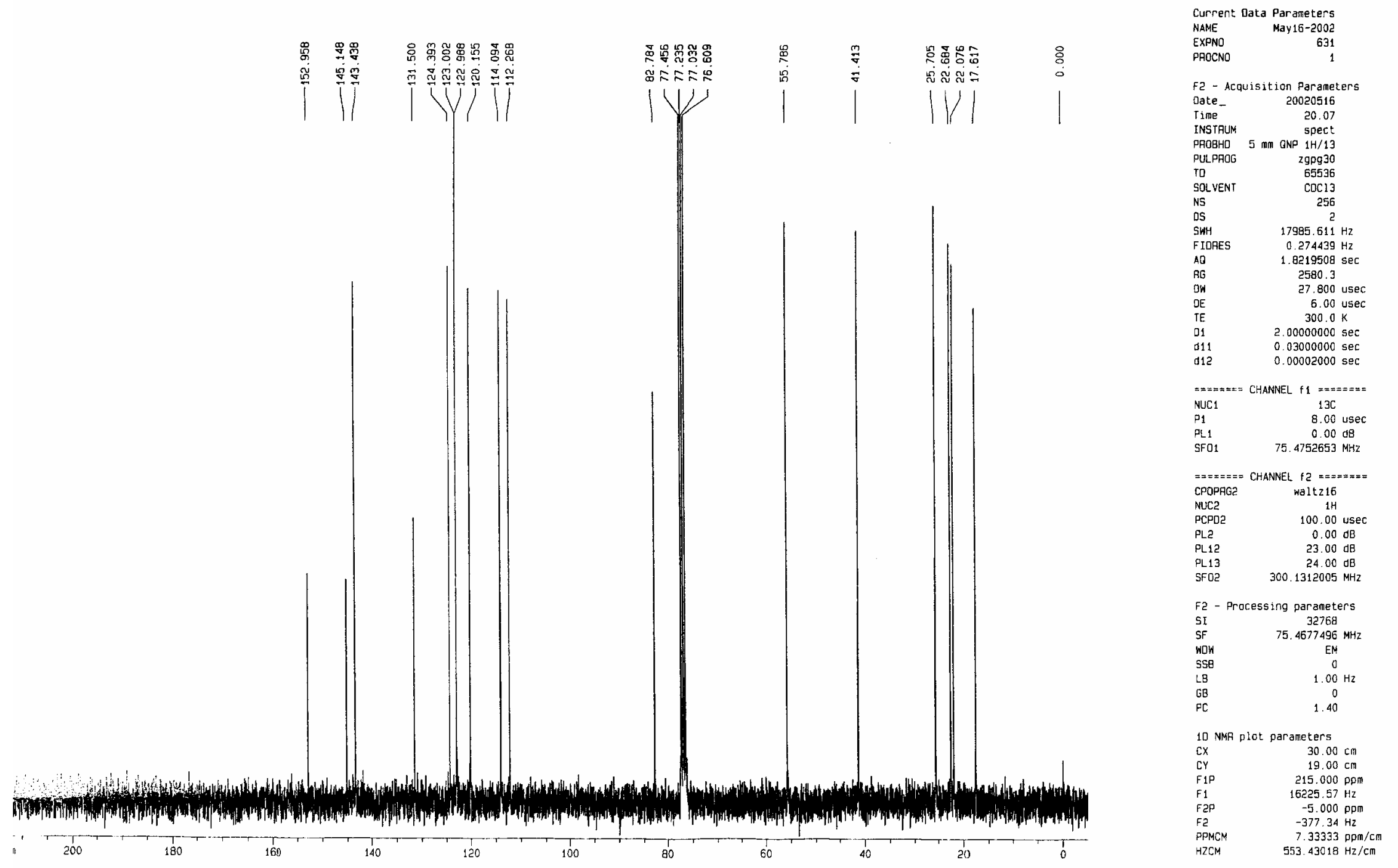
2-(3,7-Dimethylocta-2,6-dienyl)-6-methoxyphenol 16

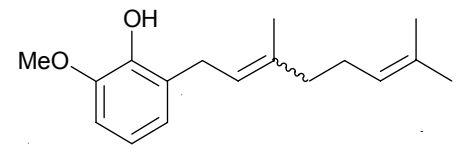

cid505ext 1

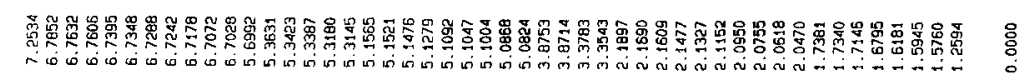
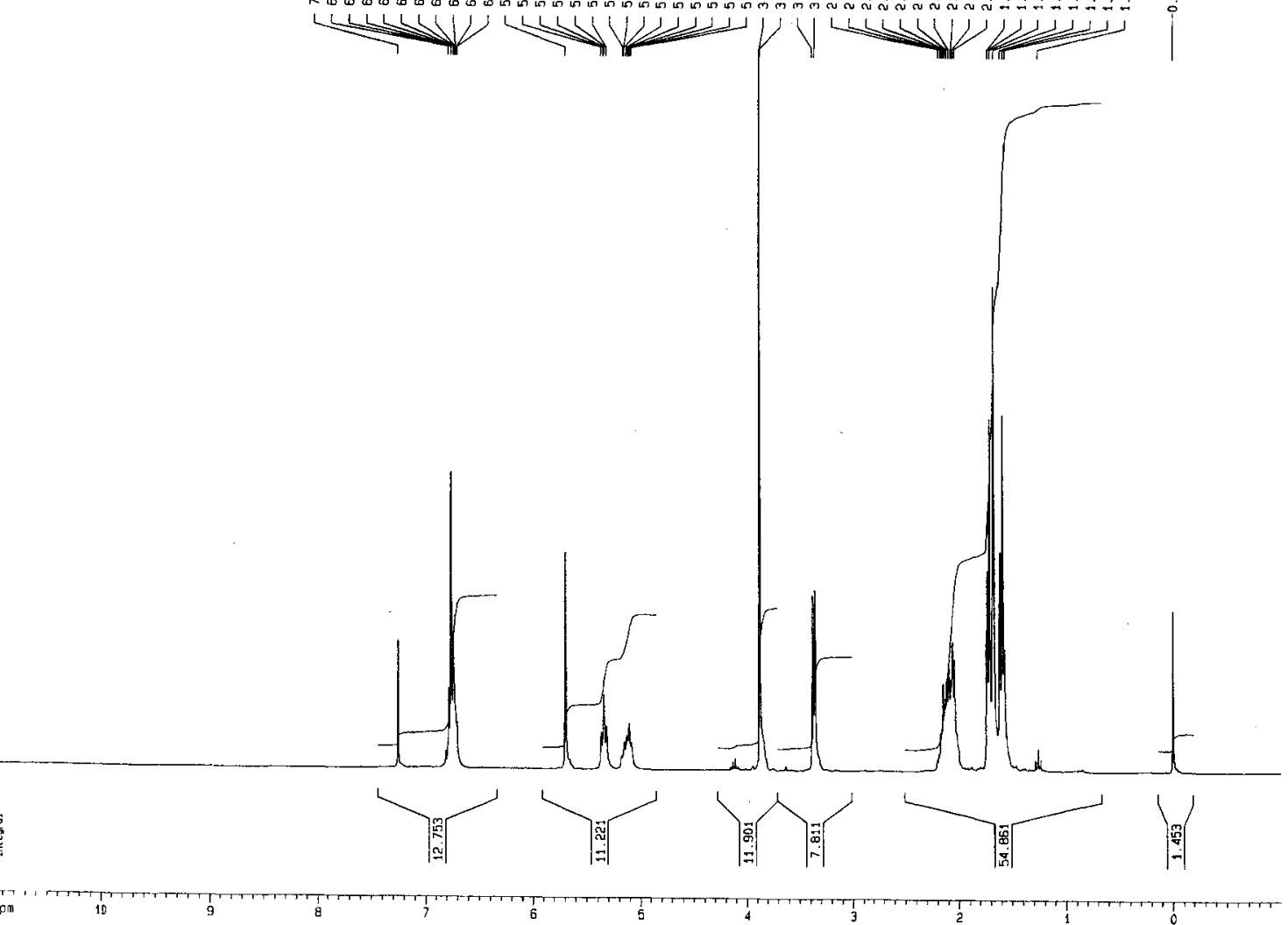

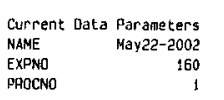

F2 - Accuisis ition Paranasers

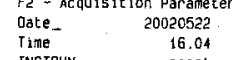

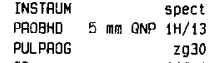

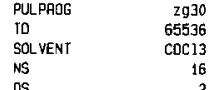

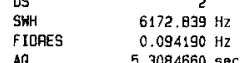

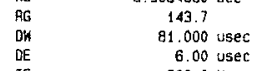

$\begin{array}{ll}T E & \begin{array}{l}3000.0 \mathrm{~K} \\ 01\end{array} \\ 01 & 1.00000000 \mathrm{sec}\end{array}$

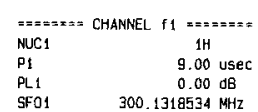

F2- Processing paraneters

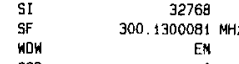

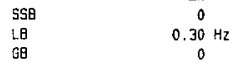

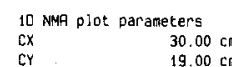

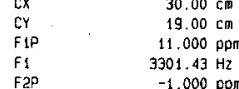

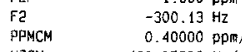

Ppacy
Hech 
2-(3,7-Dimethylocta-2,6-dienyl)-6-methoxy-1,4-benzoquinone (Verapliquinones A and B) 5/6

CJD 506

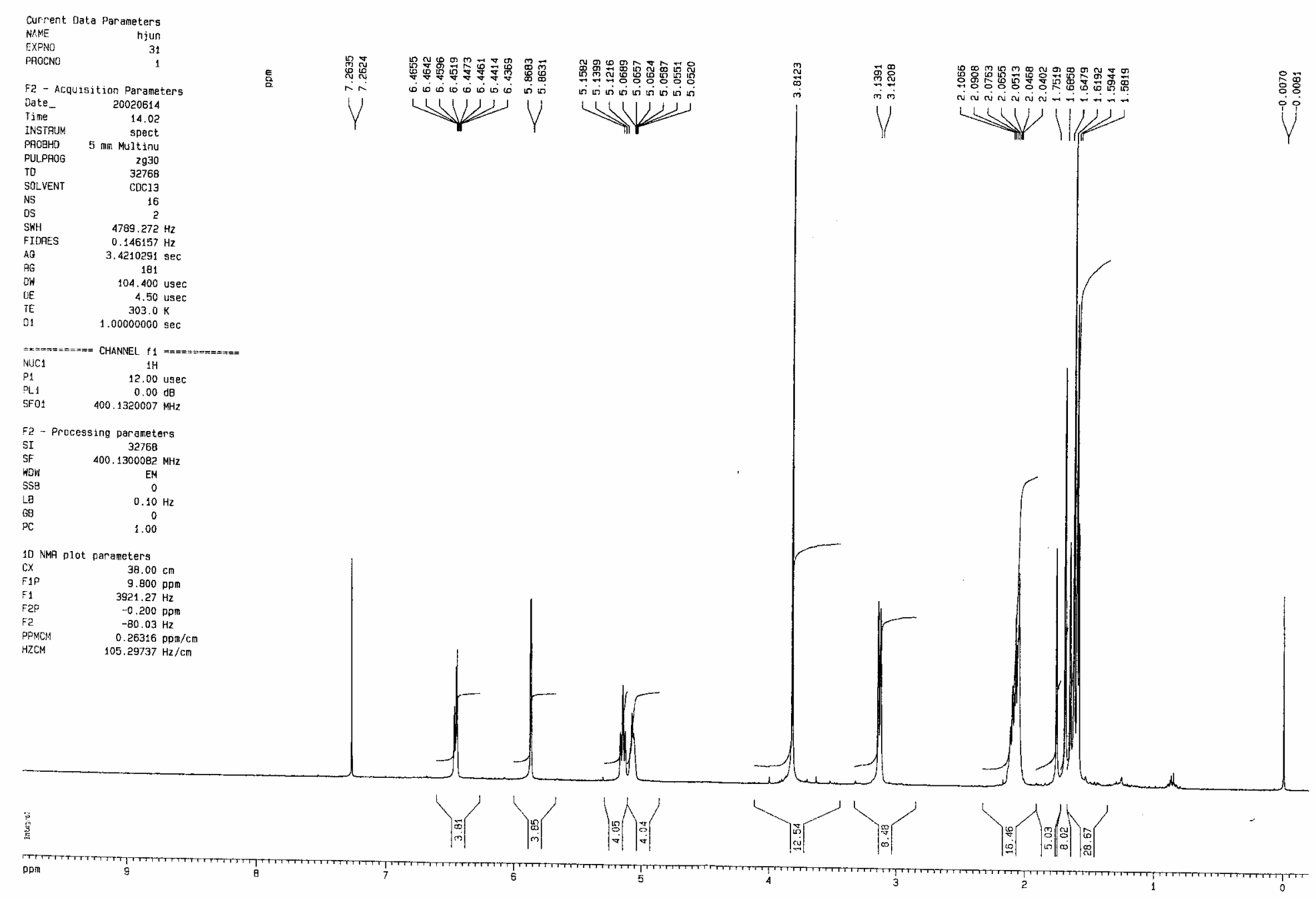


5-(4-Methoxy-2,3,6-trimethylphenyl)-3-methylpentyn-3-ol 17

TH2/24/2
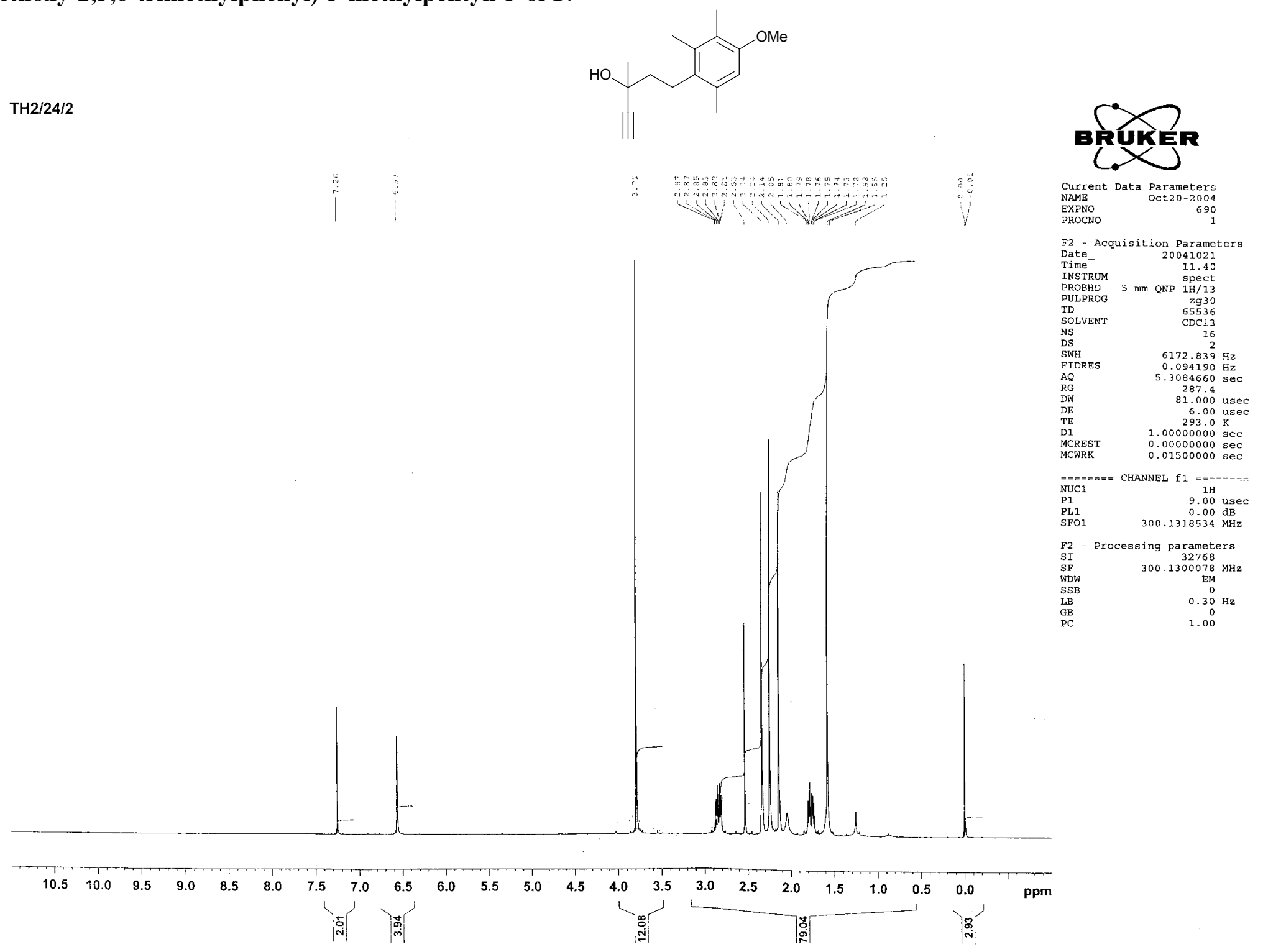
lab book ref TH2/24/2

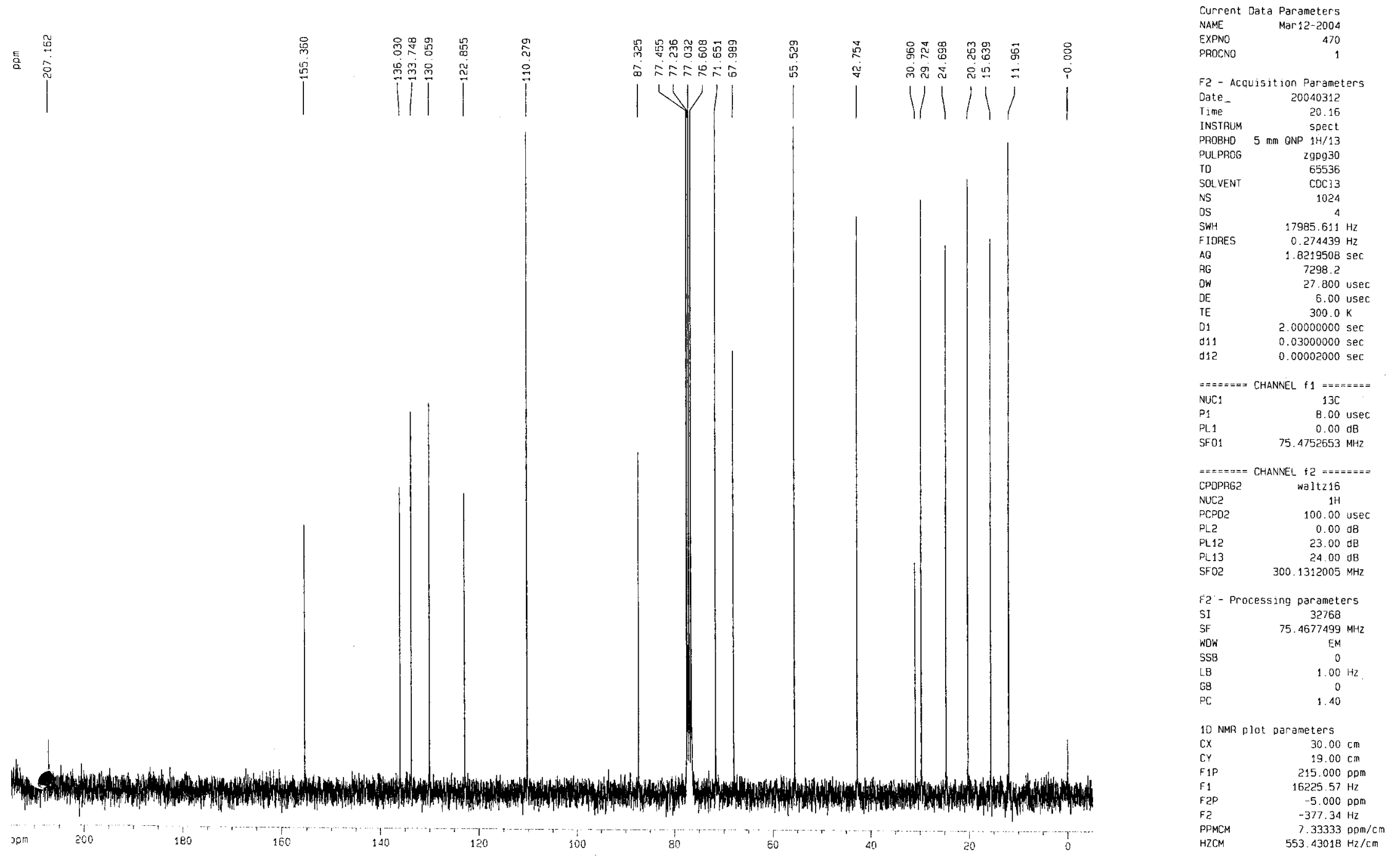


Carbonic acid 1-[2-(4-methoxy-2,3,6-trimethyl-phenyl)-ethyl]-1-methyl-prop-2-ynyl ester methyl ester

TH2/58/1
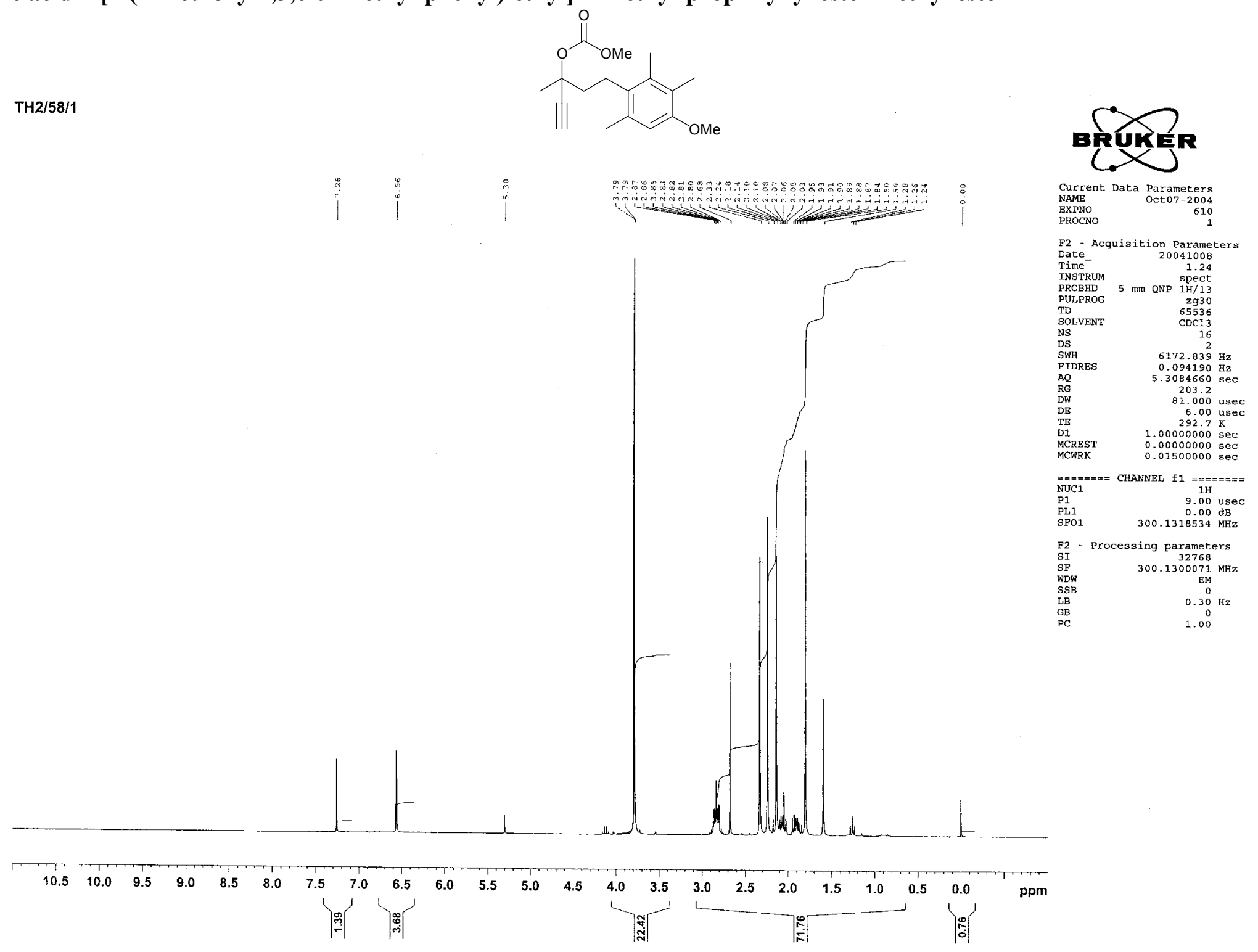

\section{BRUKER

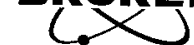

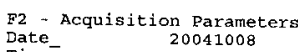

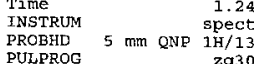

Tolve

os

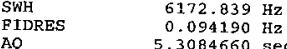

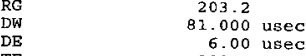

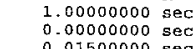

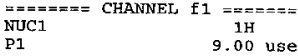

300.1318.0534 ํㅐ

SI - processing paranaterers

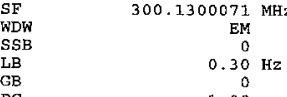




\section{BRUKER}

(x)

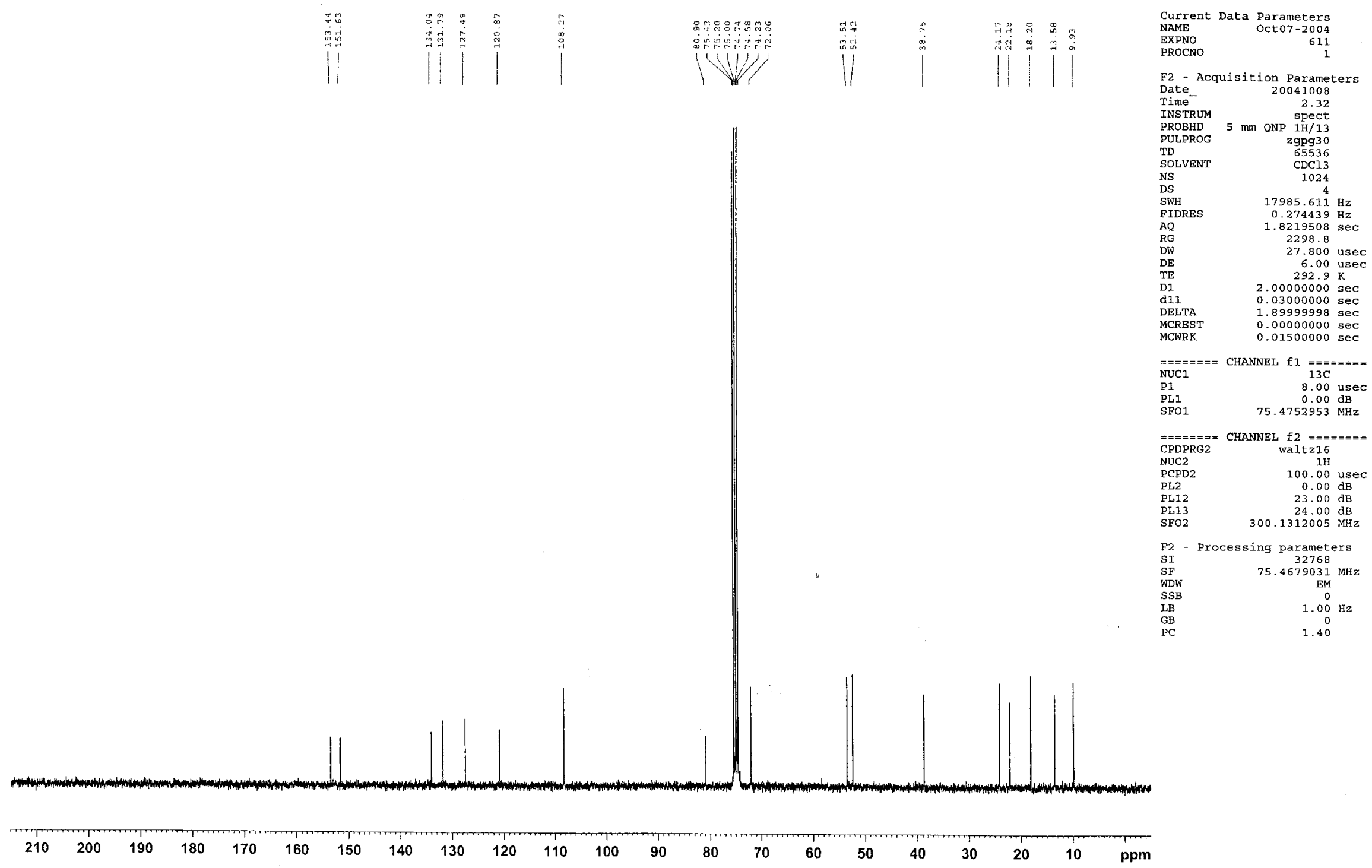


5-(4-Methoxy-2,3,6-trimethylphenyl)-3-(4-methoxyphenoxy)-3-methylpentyne 18

TH2/66/2

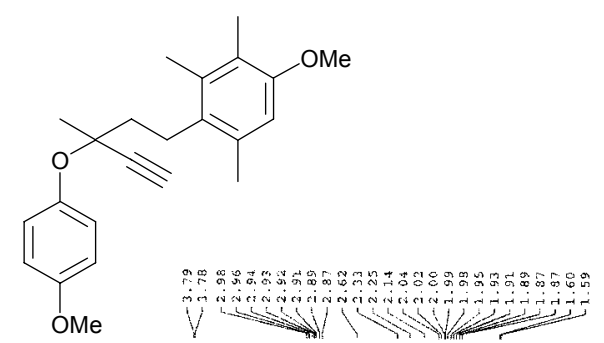

\section{BRuker \\ C.>}

Anctions

$\mathrm{OMe}$

$\begin{array}{ll}\text { Current Data } & \text { Parameters } \\ \text { NAME } & \text { OCt } 04-2004 \\ \text { EXPNO } & 10 \\ \text { PROCNO } & 10\end{array}$

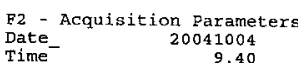

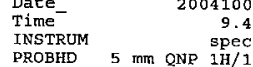

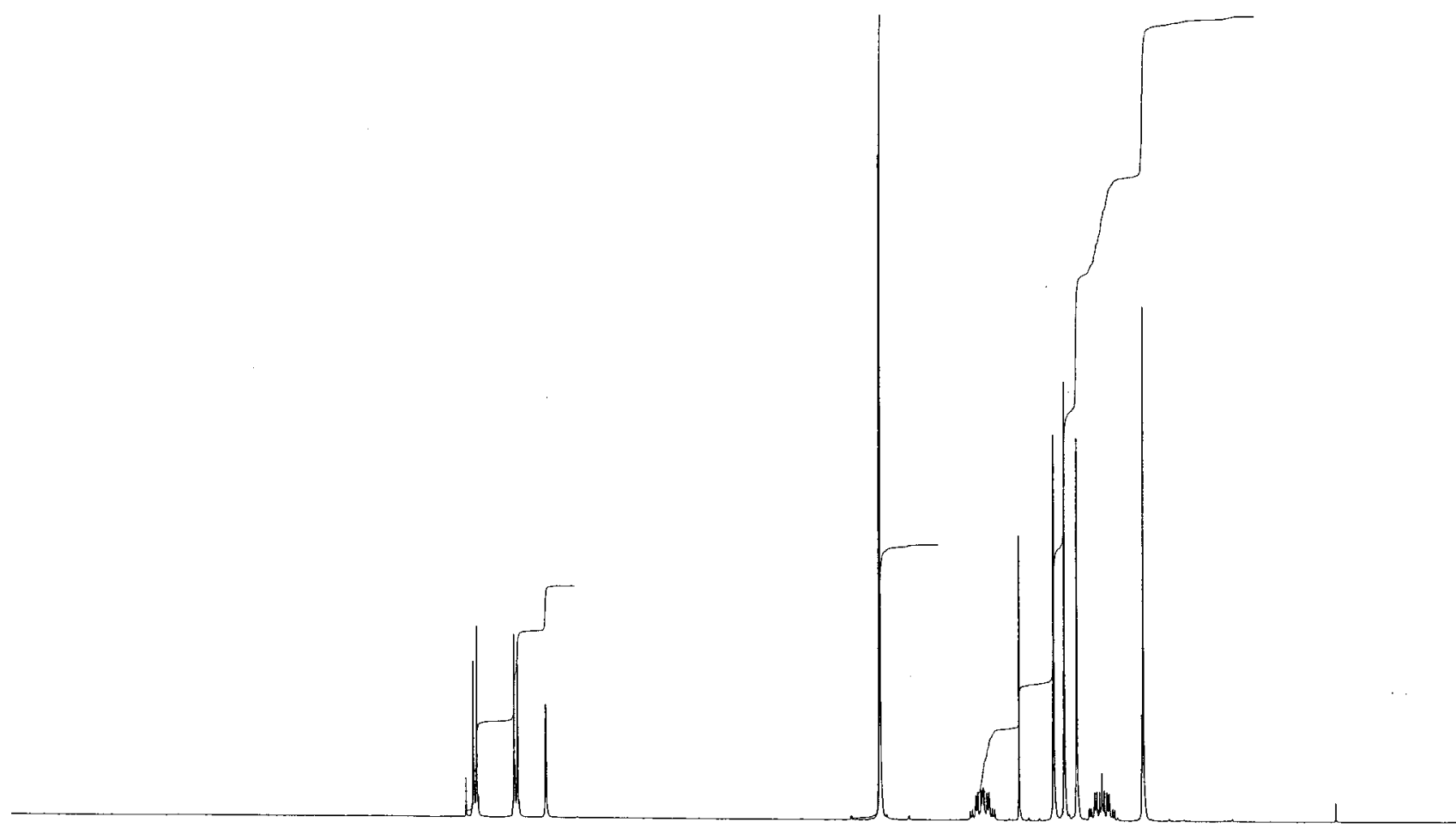

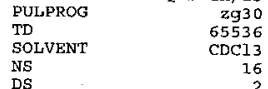

$\begin{array}{lr}\text { DS } & 2 \\ \text { SWH } & 6172.839 \mathrm{~Hz} \\ \text { FIDRES } & 0.094190 \mathrm{~Hz} \\ \text { AD } & 5.3084660 \mathrm{He}\end{array}$

$\begin{array}{lr}\mathrm{AO} & 5.3084660 \mathrm{sec} \\ \mathrm{RG} & 114 \\ \mathrm{DW} & 81.000 \mathrm{usec}\end{array}$

$\begin{array}{lr} & 114 \\ \mathrm{DW} & 81.000 \mathrm{usec} \\ \mathrm{DE} & 6.00 \mathrm{usec} \\ \mathrm{TE} & 291.0 \mathrm{~K} \\ \mathrm{TE} & 1.00000000 \mathrm{sec} \\ \mathrm{D1} & 0.0950000 \mathrm{~s}\end{array}$

$\begin{array}{ll}\text { DI } & 1.00000000 \mathrm{sec} \\ \text { MCEST } & 0.0000000 \mathrm{sec} \\ \text { MCWRK } & 0.01500000 \mathrm{sec}\end{array}$

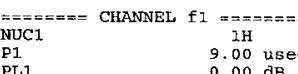

$\begin{array}{lr}\text { P1 } & 9.00 \text { usec } \\ \text { PL1 } & 0.00 \mathrm{dSB} \\ \text { SEO1 } & 300.1318534 \mathrm{MHz}\end{array}$

F2 - Processing parameters
SI
SI768
$300.1300158 \mathrm{MH}$

$\begin{array}{lc}\text { WDW } & \mathrm{EM} \\ \mathrm{SSB} & 0 \\ \mathrm{LB} & 0.30 \mathrm{~Hz} \\ \mathrm{LB} & 0 \\ \mathrm{~GB} & 1.00\end{array}$
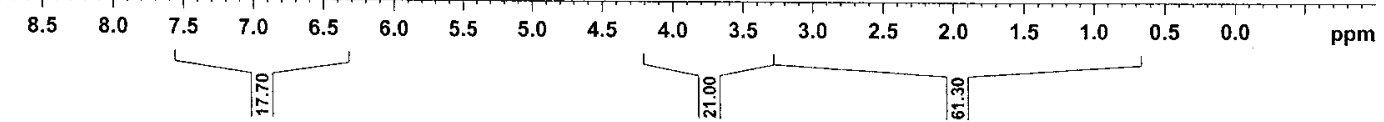


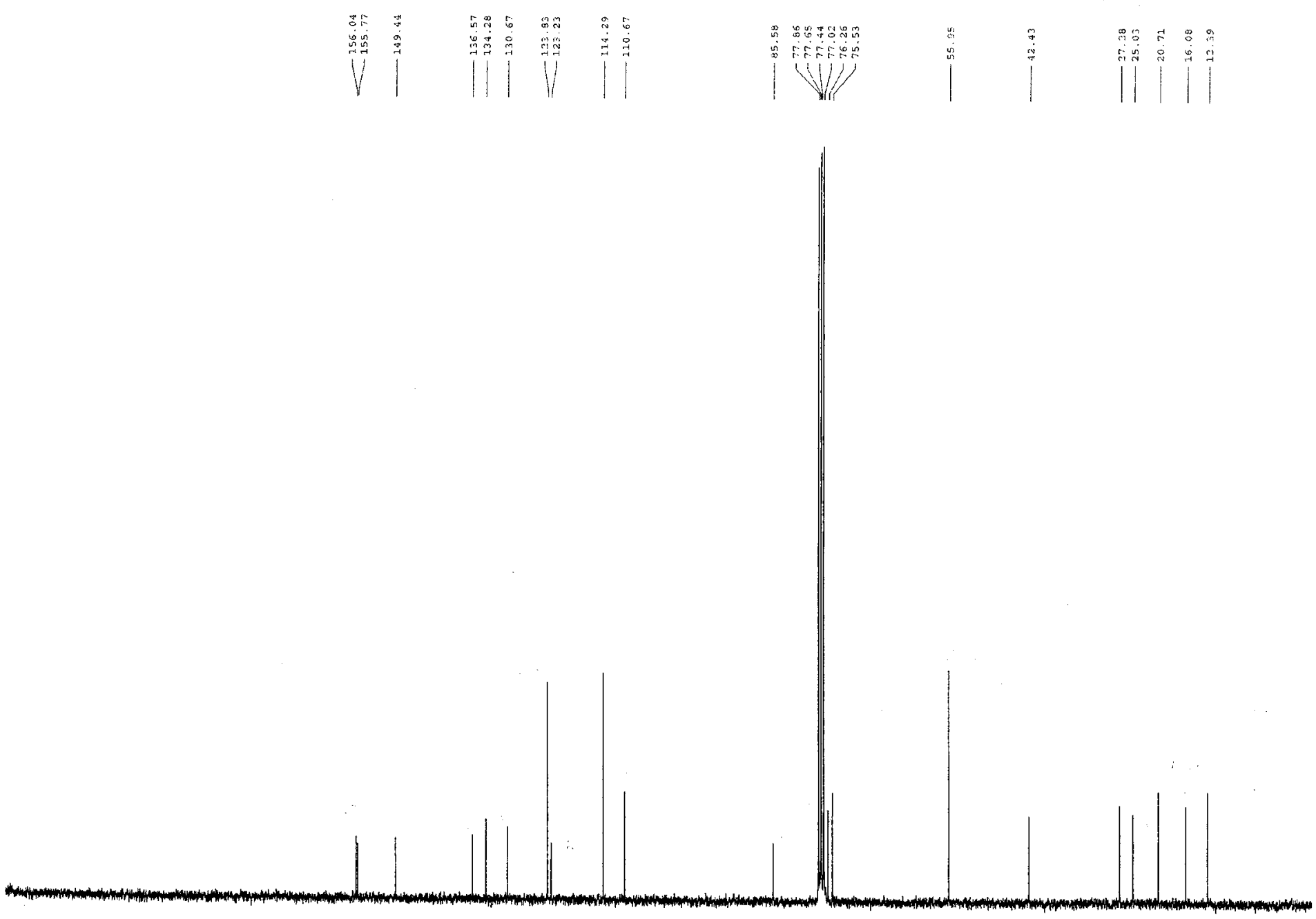


5-(4-Methoxy-2,3,6-trimethylphenyl)-3-(4-methoxyphenoxy)-3-methylpentene 19

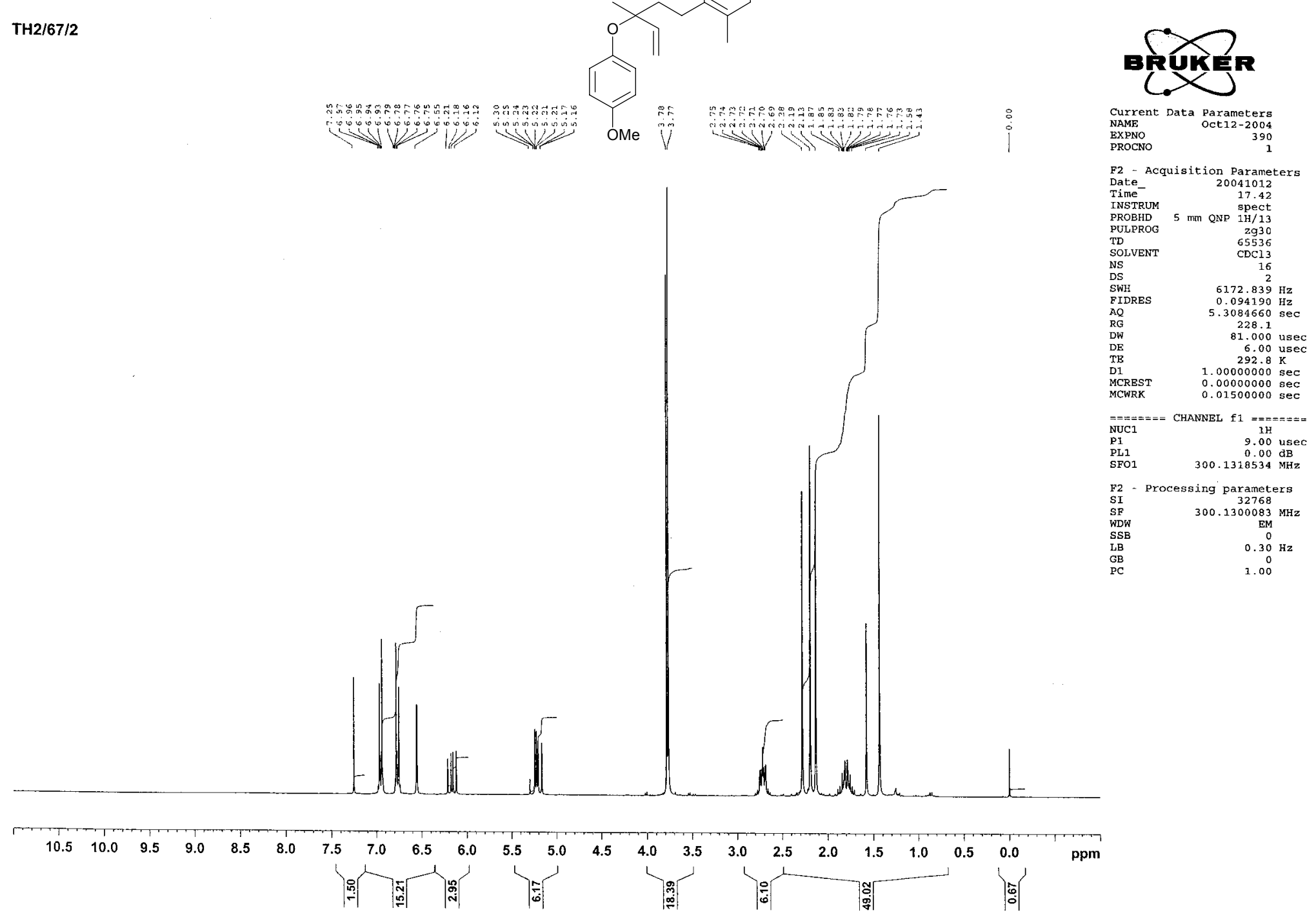




\section{BRUKER}
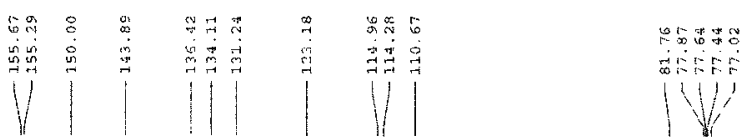

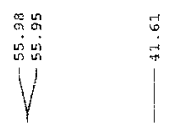
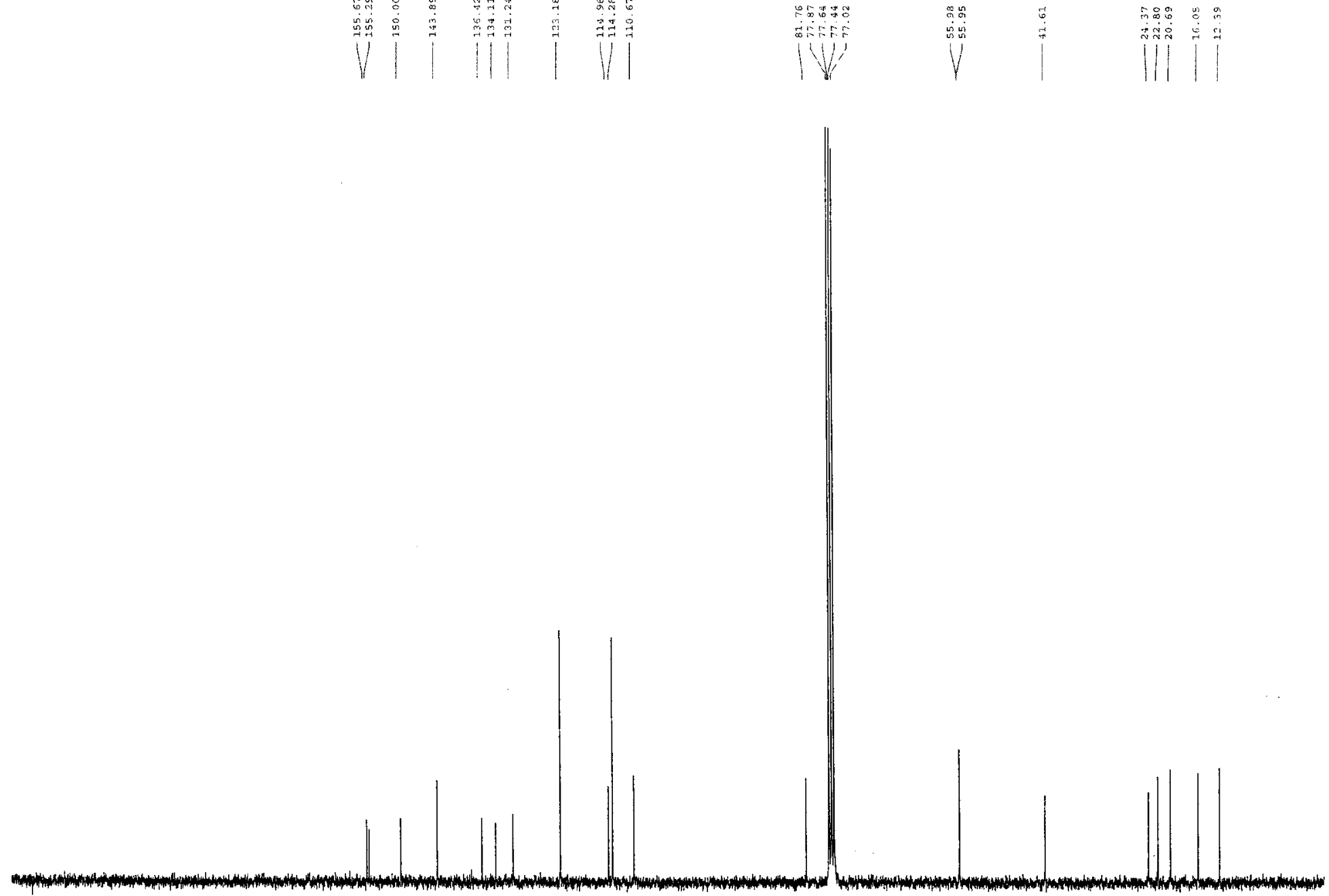

mons 
4-Methoxy-2-[5-(4-methoxy-2,3,6-trimethylphenyl)-3-methylpent-2-enyl]phenol 20

TH2/68/1

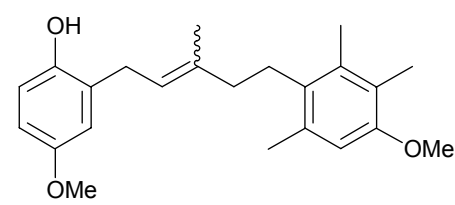

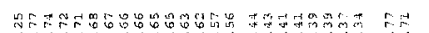
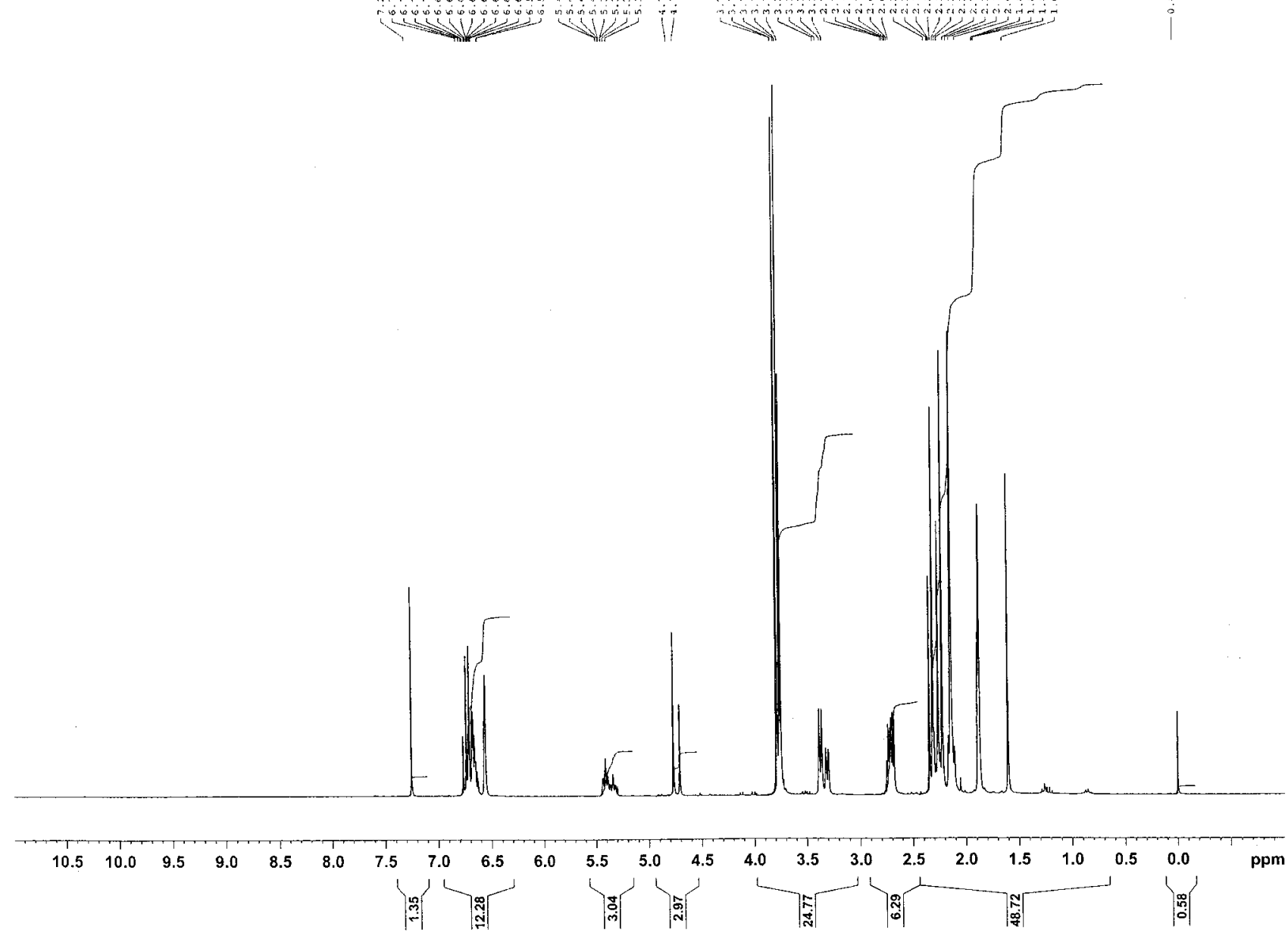

\section{BRYRer}

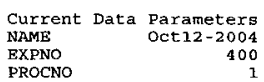

F2- Acquisition parameters
Date
Doc41012

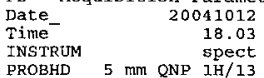

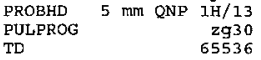

$\underset{\substack{\text { Solver } \\ \text { Ns }}}{\text { s. }}$

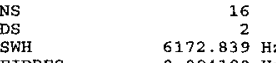

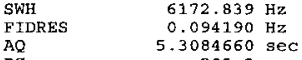

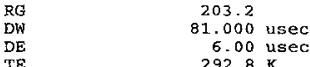

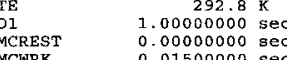

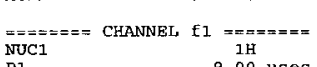

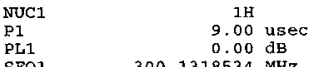

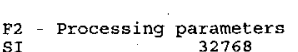

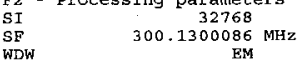

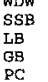

.30 Bz 


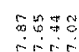

(i)

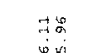

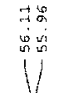
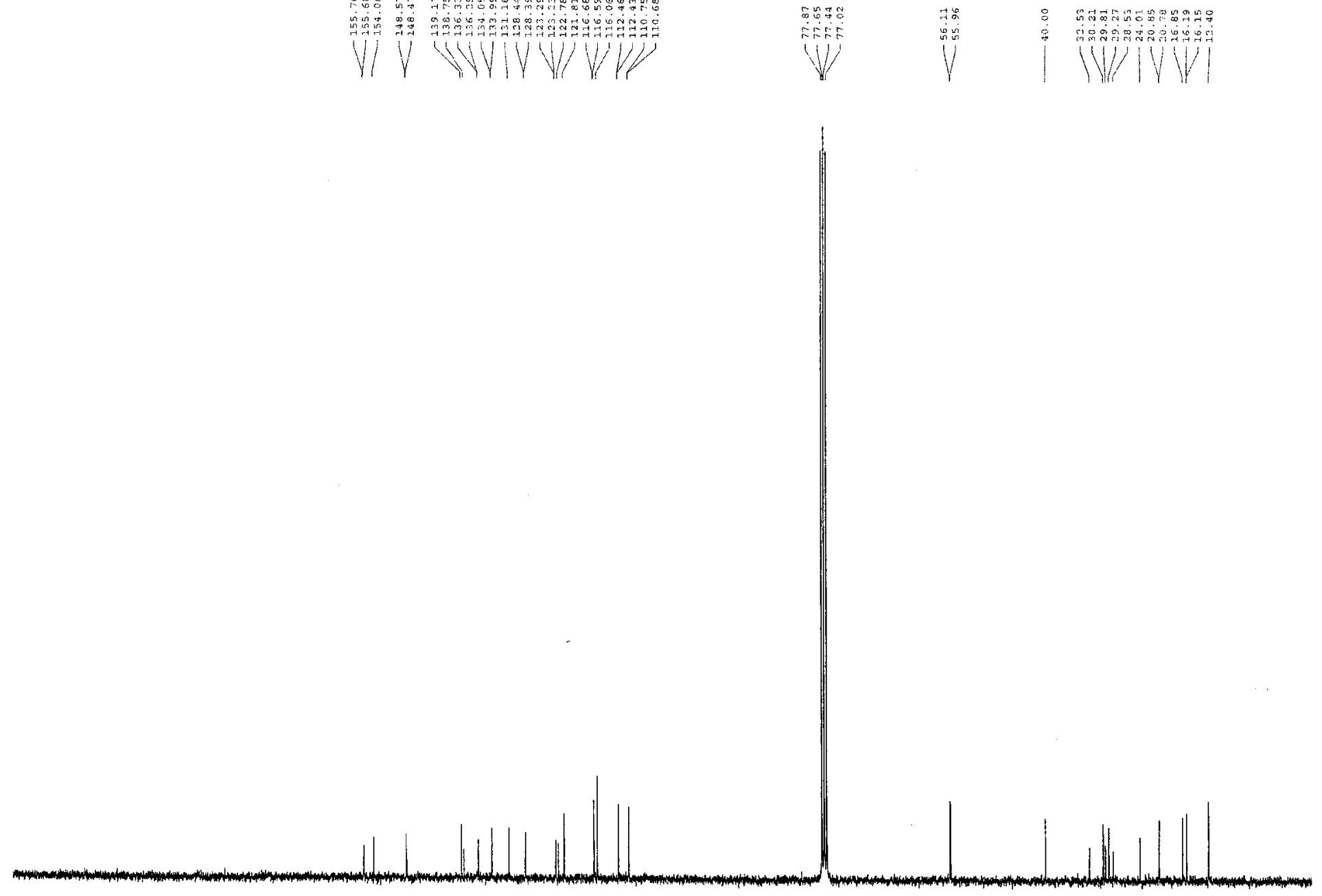

\section{cox}

Current Data Parameter

ERPNO
RROCNO

F2 - Acquisition Parameter

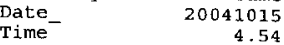

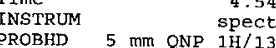

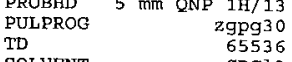

SOL
NS
DS

1024

$\begin{array}{ll}\quad 17985.611 \mathrm{~Hz} \\ \text { FIDRES } & 0.274439 \mathrm{~Hz} \\ \text { AQ } & 1.8219508 \mathrm{sec}\end{array}$

$\begin{array}{ll}\mathrm{AQ} & 1.8219508 \mathrm{sec} \\ \mathrm{RG} & 2048\end{array}$

27.800 usec
DW
DE

D1 2.0000000 sec

d11 $\quad 0.030000000 \mathrm{sec}$

$\begin{array}{ll}\text { DELTA } & 1.89999998 \mathrm{sec} \\ \text { MCREST } & 0.00000000 \\ \text { MCWRK } & 0.01500000 \text { sec }\end{array}$

$==\pi=-=-=$ CHANNEL f1 $== \pm===-$

$\begin{array}{lr}\text { NUC1 } & 13 \mathrm{C} \\ \text { P1 } & 8.000 \text { usec } \\ \text { PL1 } & 0.000 \text { dB } \\ \text { SFO1 } & 75.4752953 \mathrm{MHz}\end{array}$

$=======$ CHANNEL $f 2=======$
CPDPRG 2 .

$\begin{array}{ll}\text { NUC2 } & \text { waltz16 } \\ \text { NPDD2 } & 100.00 \text { usec } \\ \text { PT2 } & 10.00 \text { d }\end{array}$

$\begin{array}{ll}\text { PL2 } & 0.00 \mathrm{~dB} \\ \text { P12 } & 23.00 \mathrm{~dB} \\ \text { PL13 } & 24.00 \mathrm{~dB}\end{array}$

F2 - Processing parameters

$\begin{array}{ll}\text { SI } & 32768 \\ \text { SF } & 75.4677190 \mathrm{MH}\end{array}$

WD
$\mathrm{LS}$
$\mathrm{LB}$

GC

0
$1.00 \mathrm{~Hz}$

1.40

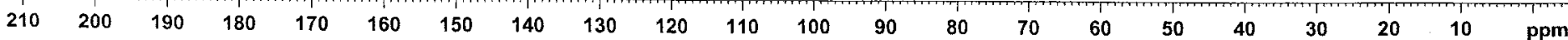


2-[5-(4-Methoxy-2,3,6-trimethylphenyl)-3-methylpent-2-enyl]-1,4-benzoquinone (Panicein A and isomer) 7

TH2/69/1
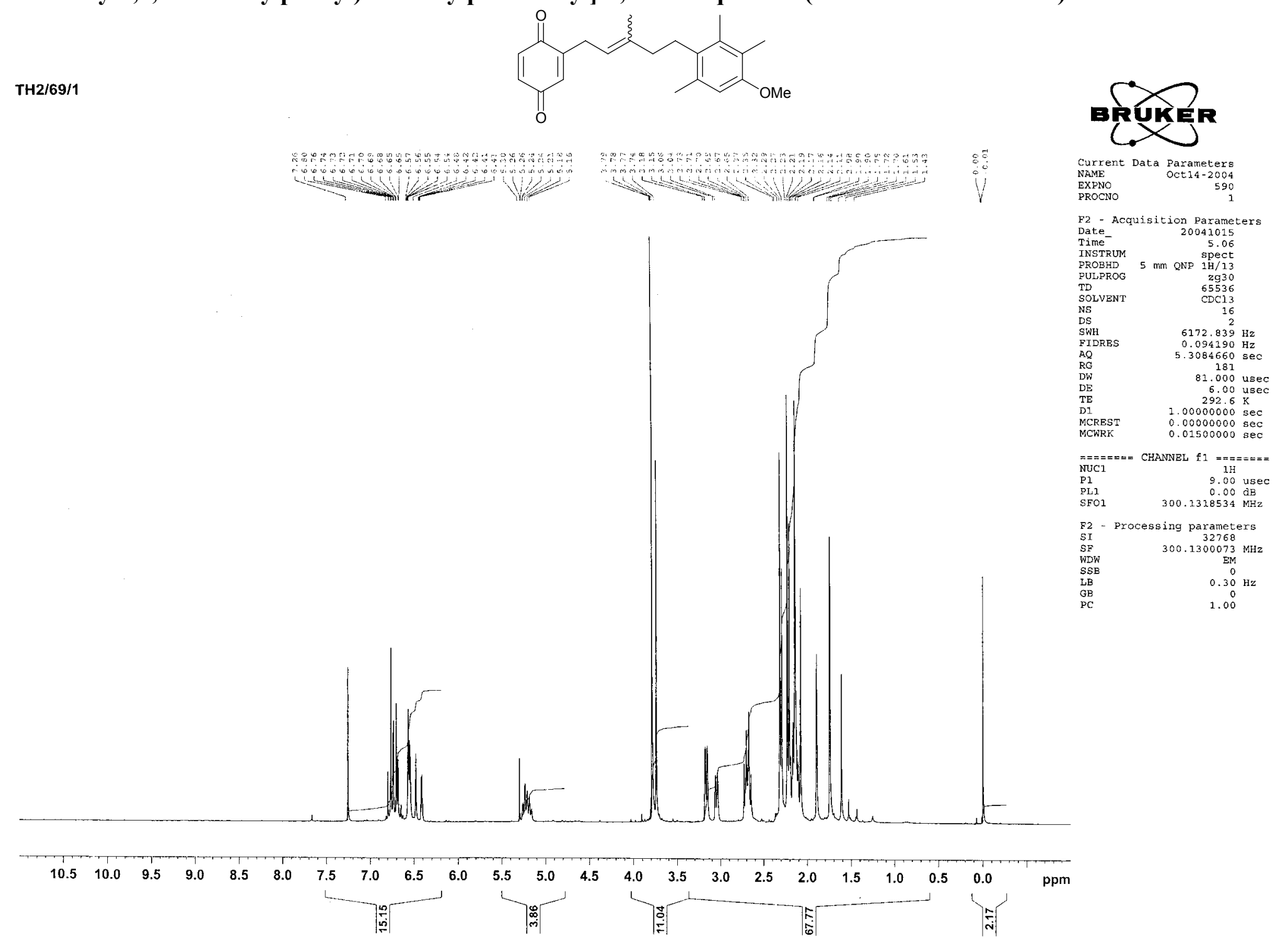

\section{BRUKER}

PROCNO

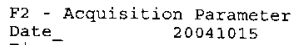

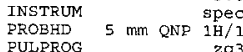

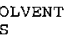

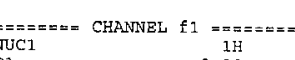

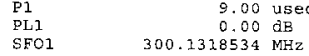

2- Processing parancters

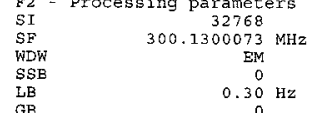


TH2/69/1

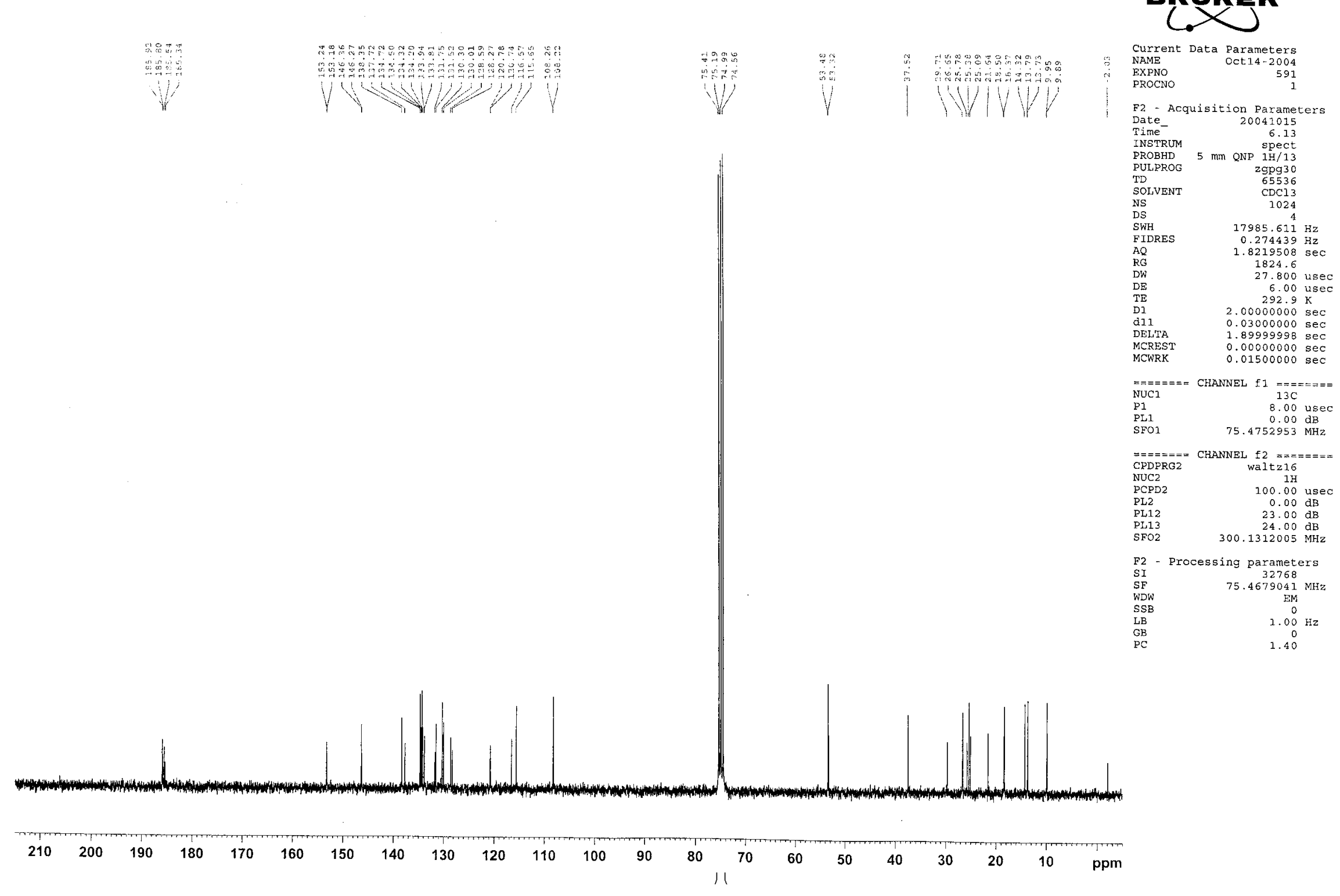

\section{BRUKER ,}

F2 - Acquisition Parameters
Date_
20041015

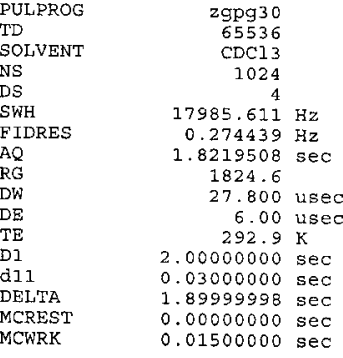

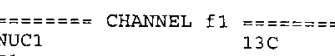
$\begin{array}{ll}8.00 \mathrm{usec} \\ \text { PL1 } & 0.00 \mathrm{~dB}\end{array}$

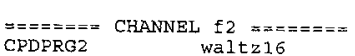
$\begin{array}{lc}\text { NUC2 } & 1 \mathrm{H} \\ \text { PCPD2 } & 100.00 \mathrm{usec} \\ \text { PL2 } & 0.00 \mathrm{~dB}\end{array}$ $3.00 \mathrm{~dB}$ soo. 1312005 Mtz F2 - Processing parameters 75.4679041 MHz $\begin{array}{ll}\mathrm{LB} & 1.00 \mathrm{~Hz}\end{array}$ 
(E)-Ethyl-3-(3-furyl)propenoate

IMJ 265
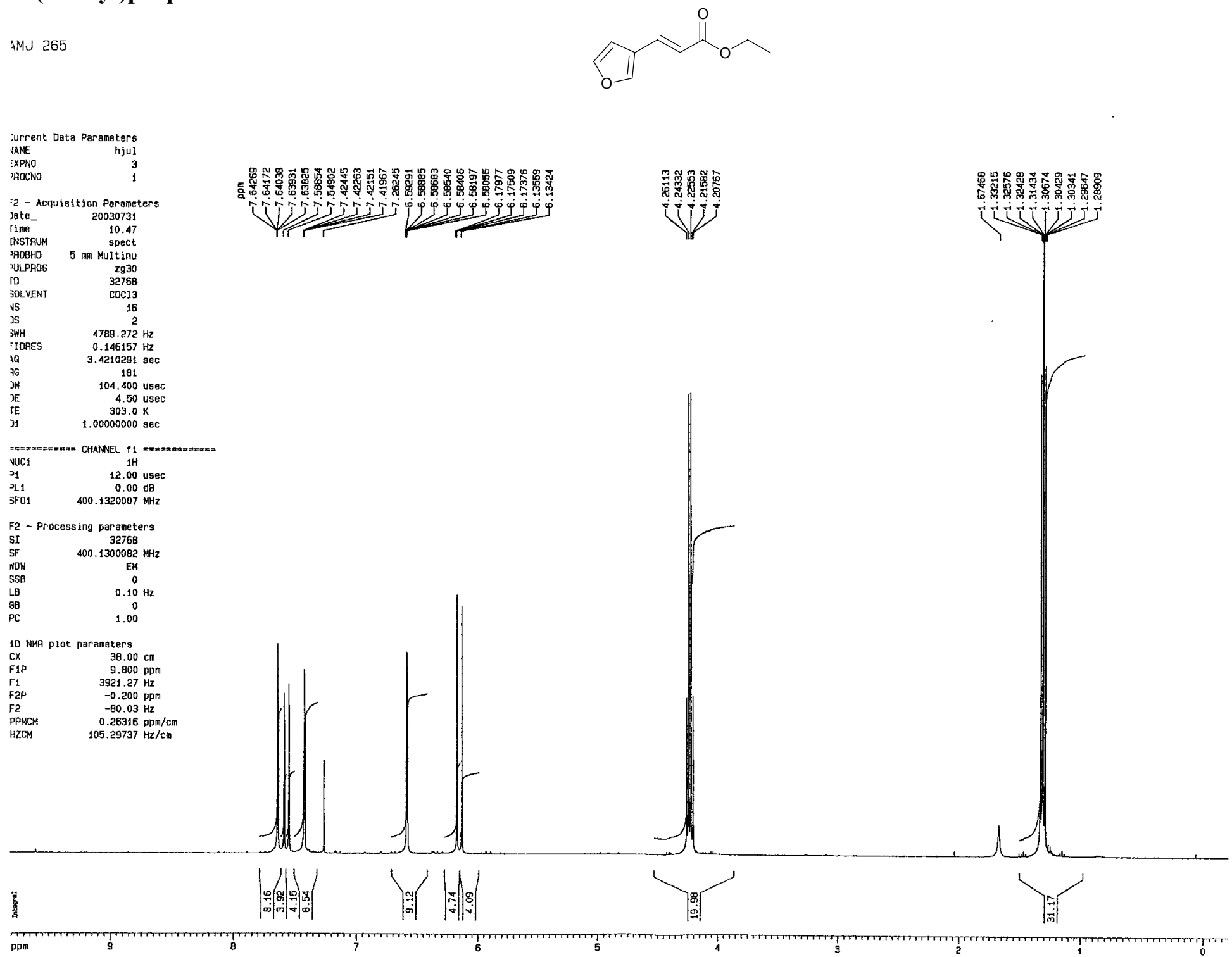
AMJ 265
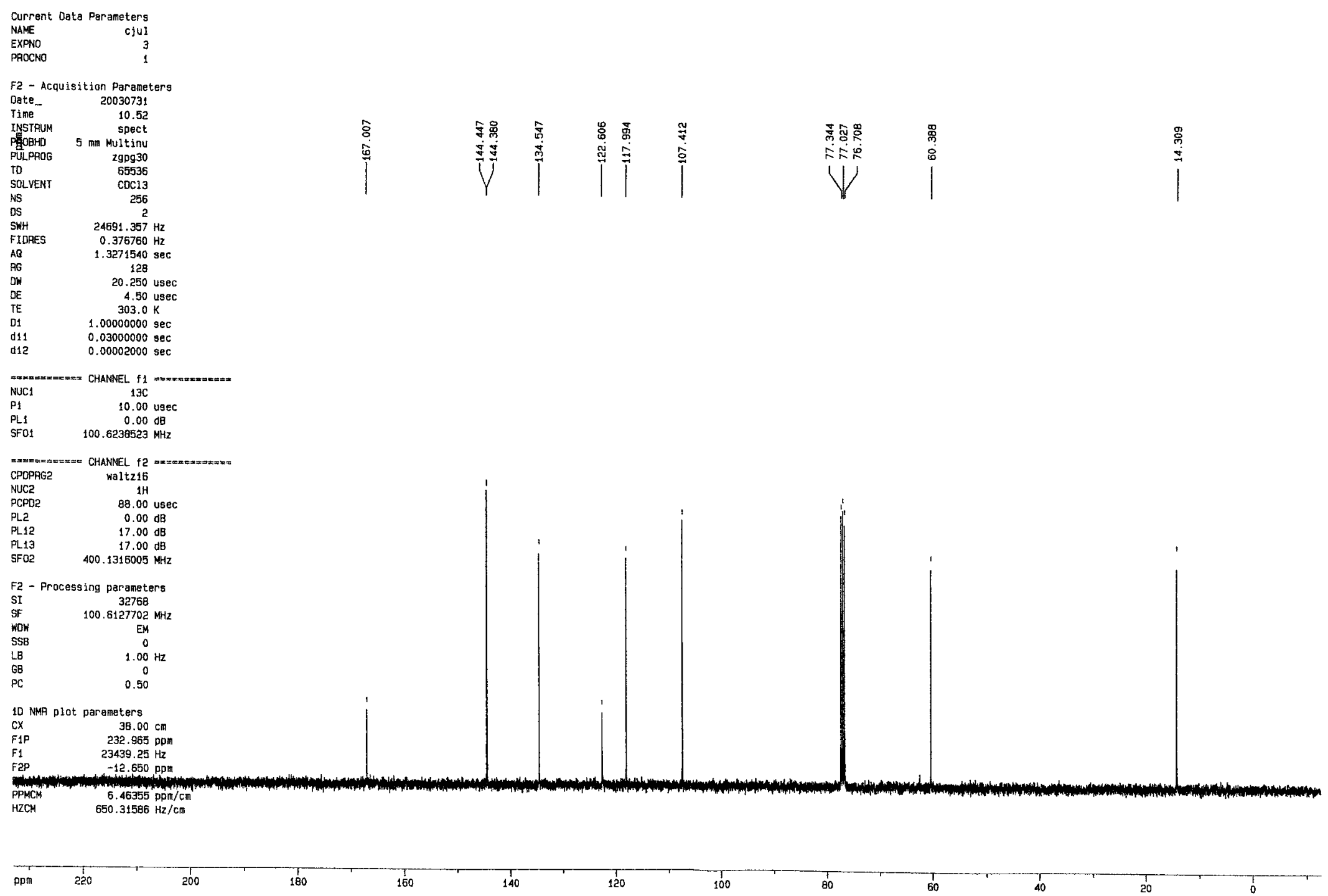
(E)-Ethyl-3-(3-furyl)propanoate

AMJ 307
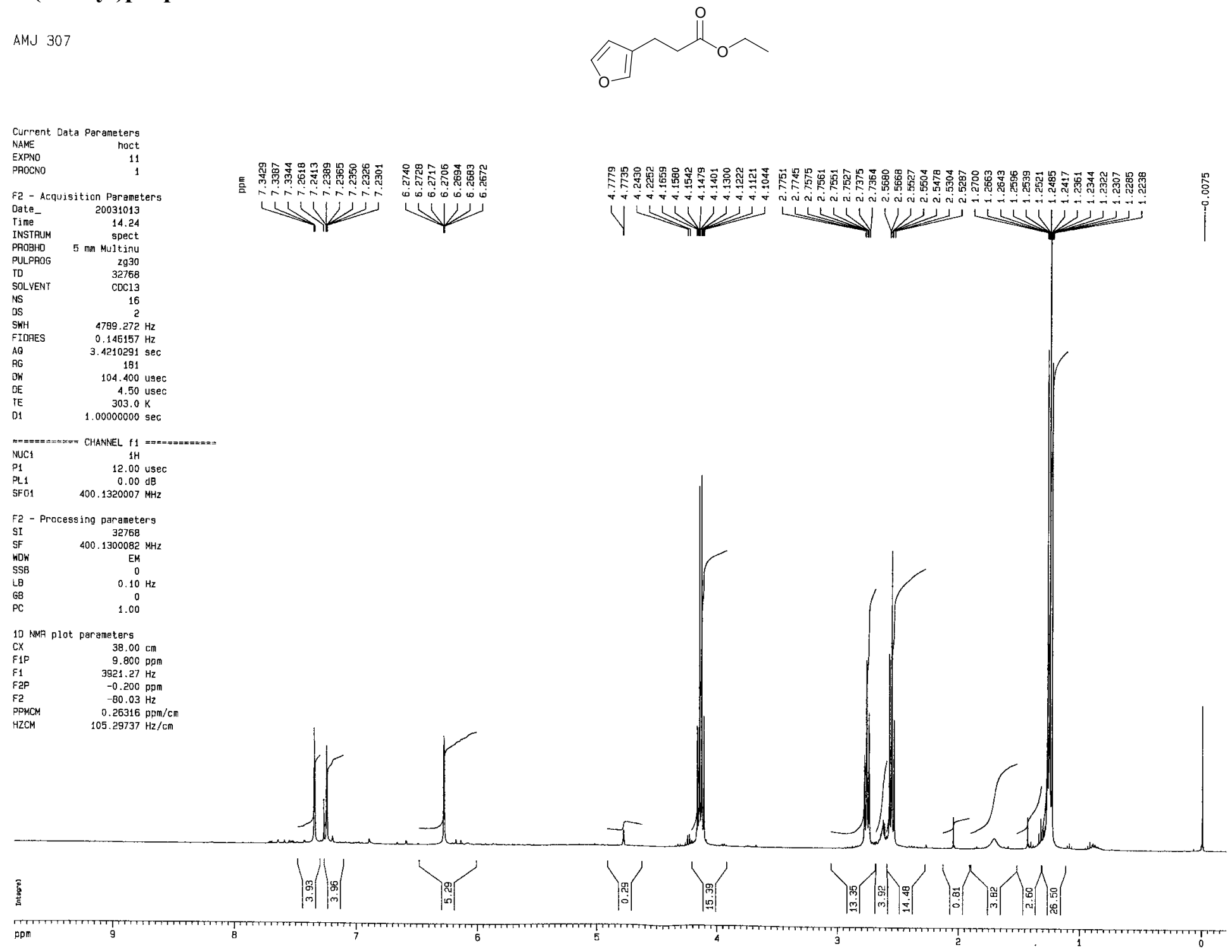

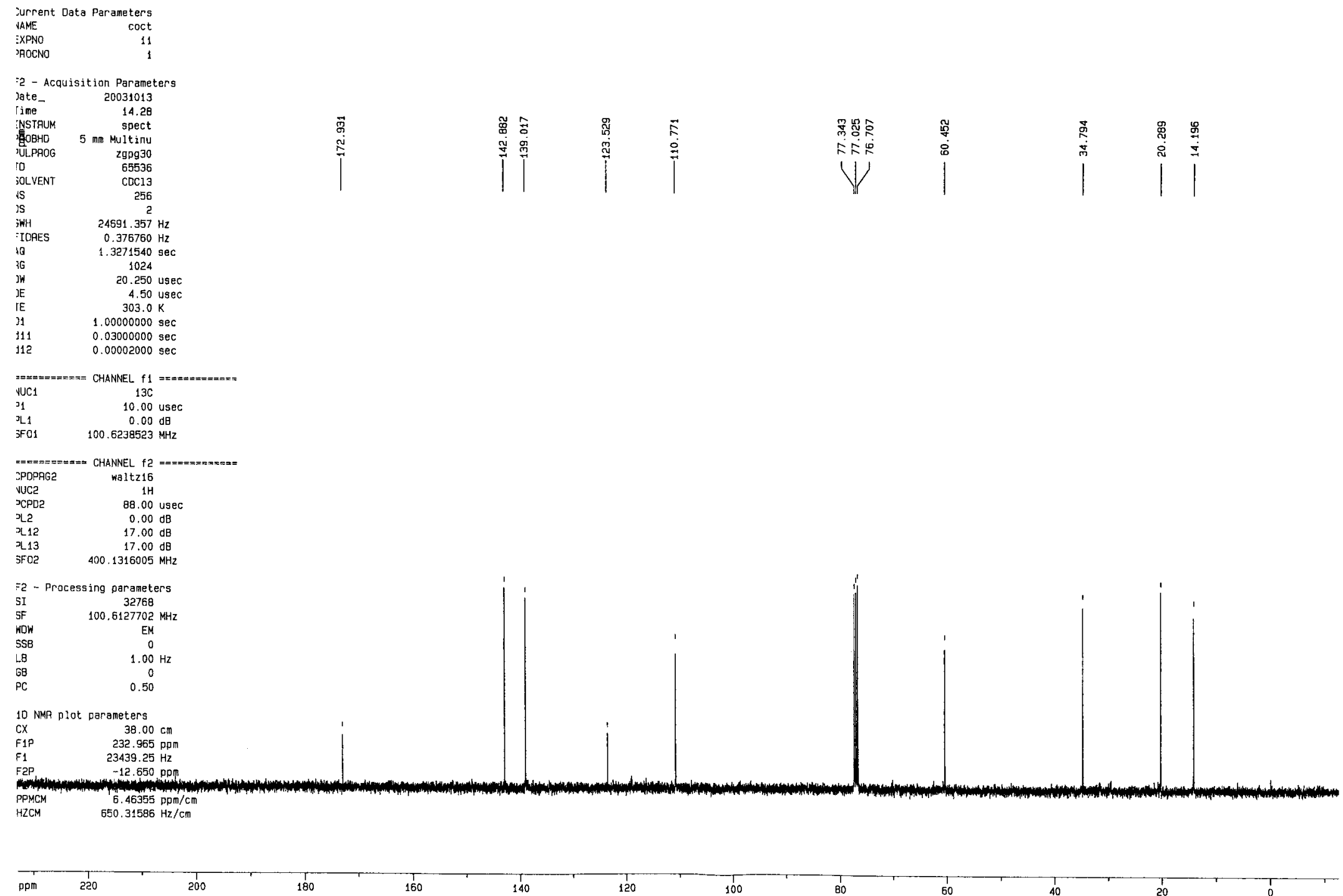
3-(3-Furyl)propanal 21

AMinj 314
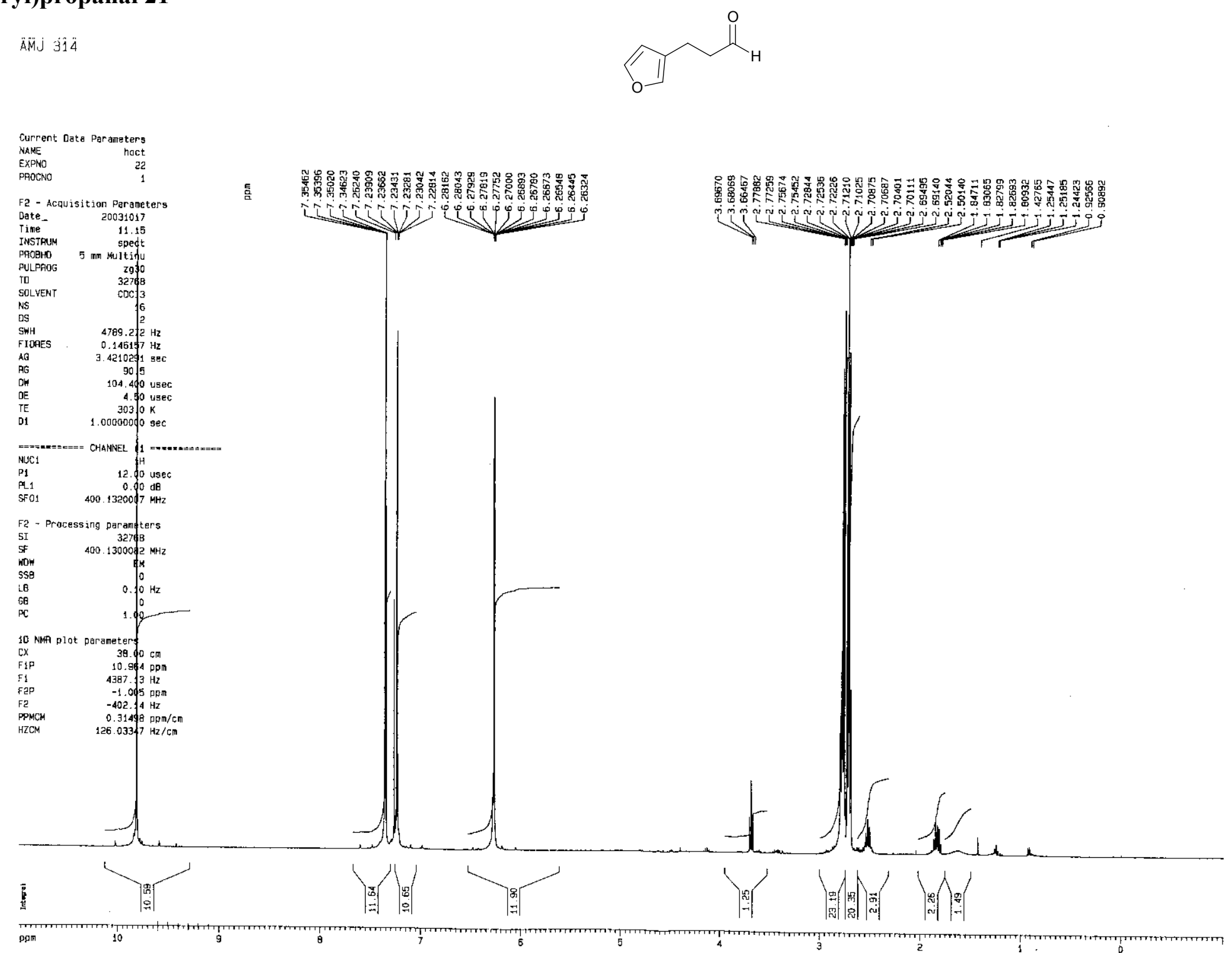
AMJ 314

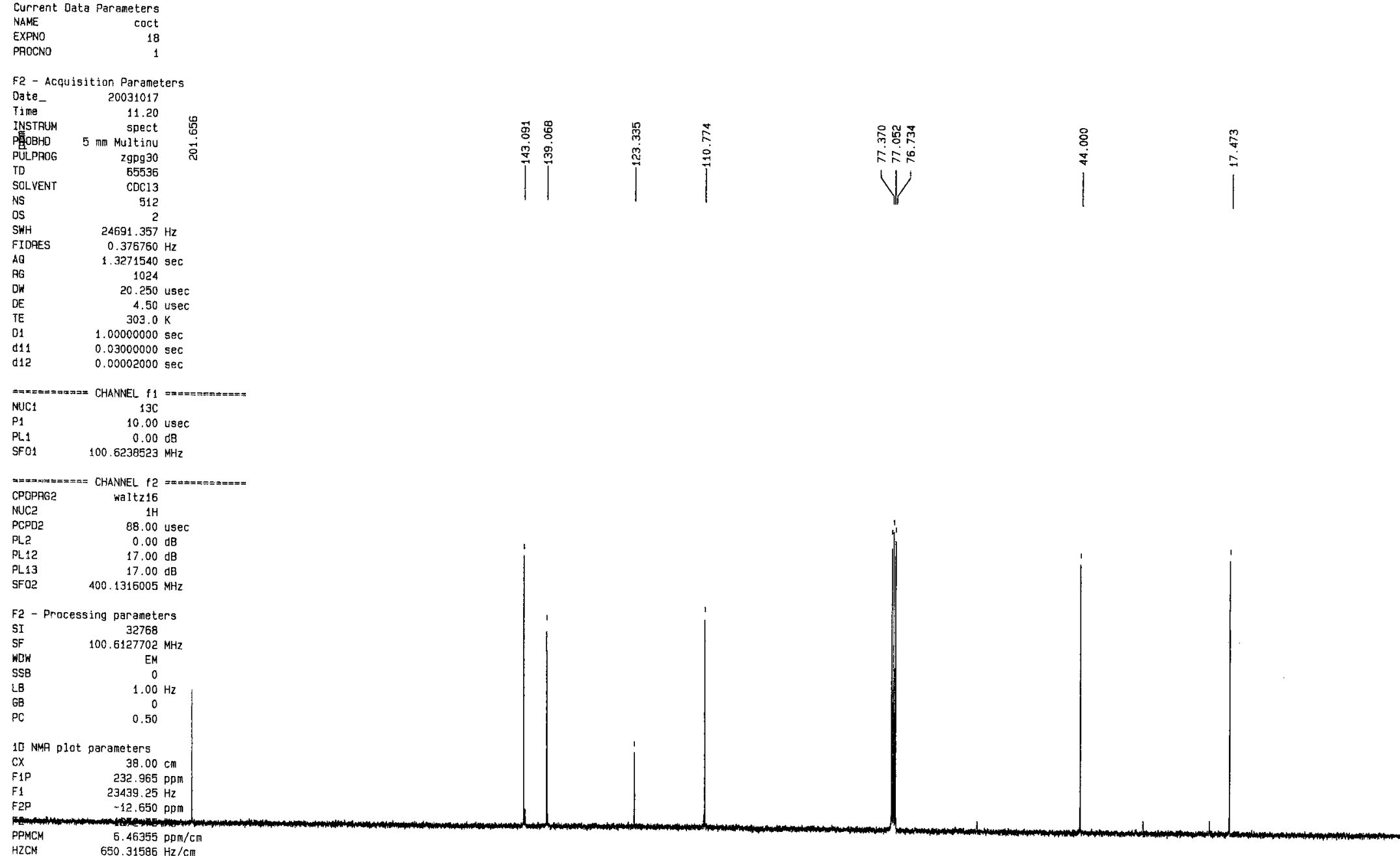

F2 - ACquisition Parameters

SOLVENT
NS

SWH $\quad 24691.357 \mathrm{~Hz}$

$\begin{array}{ll}A B & 1.3271540 \mathrm{se} \\ \mathrm{BO} & \end{array}$

DW $\quad 20.250$ us

$\begin{array}{ll}\mathrm{d} 11 & 0.03000000 \\ \mathrm{~d} 12 & 0.00002000\end{array}$

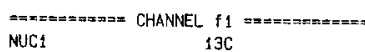

$\begin{array}{ll}\text { P1 } & 10.00 \text { usec } \\ \text { PL1 } & 0.00 \text { g }\end{array}$

CPCPPG
NHC?

$\overbrace{\text { porit }}^{120}$

$\frac{1}{200}$

${ }_{180}+160$

$\underset{140}{7}$

120

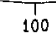

$\frac{1}{60}$

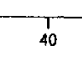

20

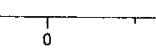


5-(3-Furyl)-2-methylpenten-3-ol 22

AMJ 320
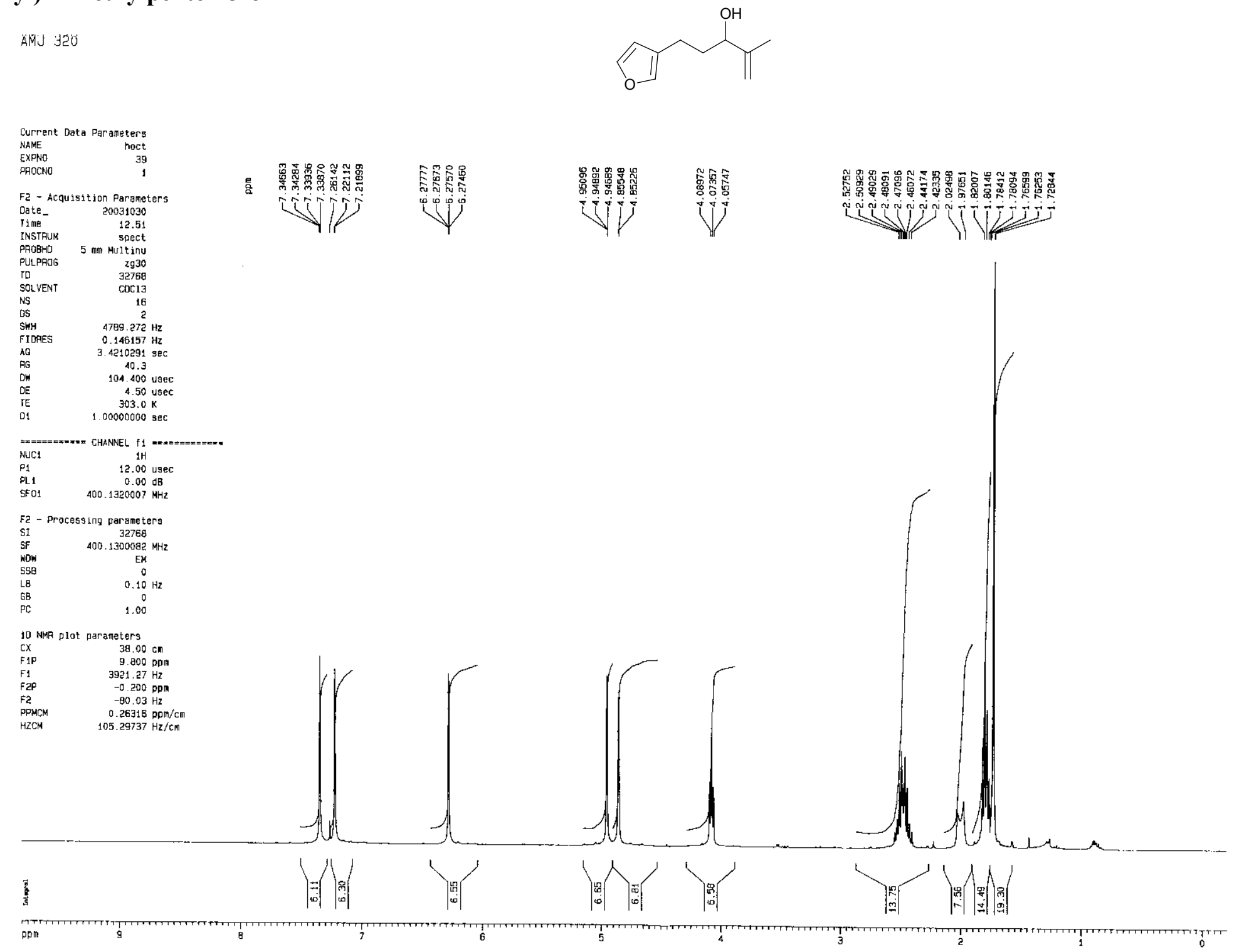
ĀMus seu

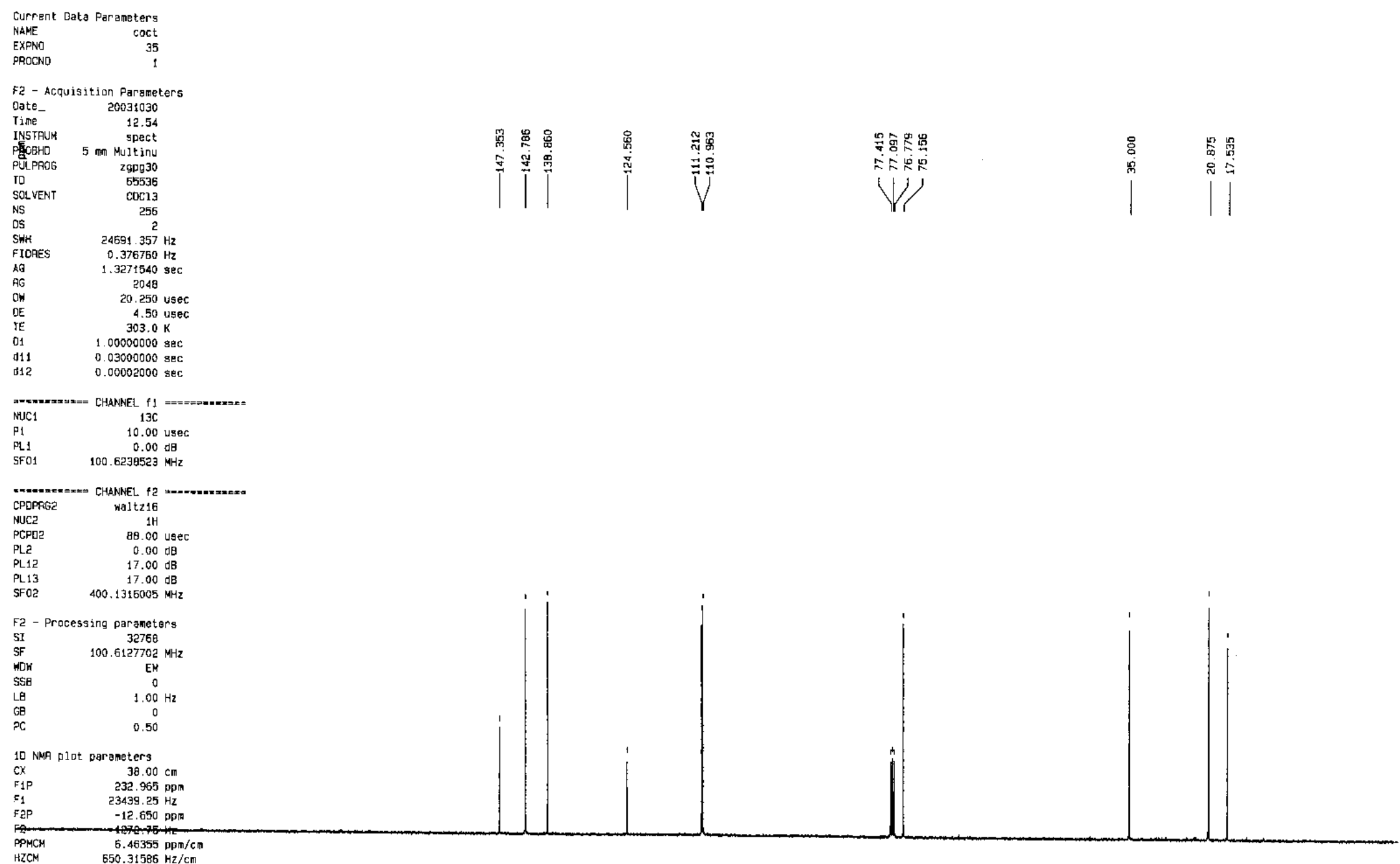

200

160

1 140

120

$\sqrt{100}$

60

40

. 30 $-$ 
3-[3-(2,3-Dimethoxy-phenoxy)-4-methyl-pent-4-enyl]-furan 23

IMJ 322

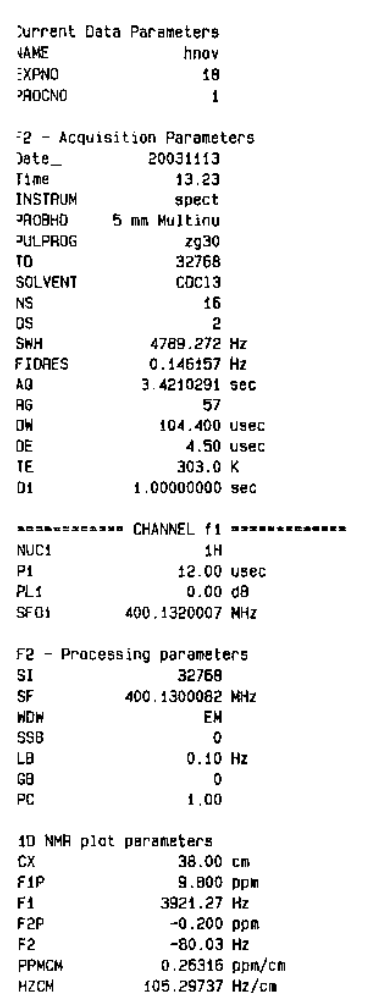

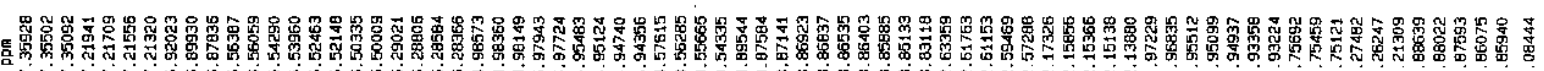

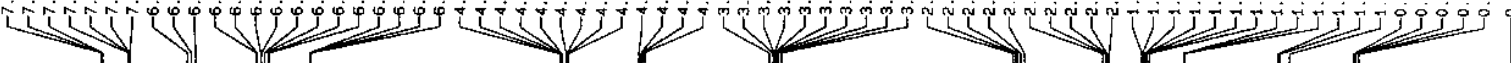

M 13.2

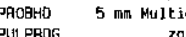

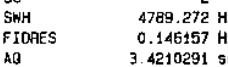

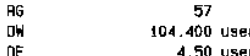

NUC1

$490 .+30.000789$

Fe - Pracess ing paraneterent

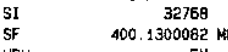

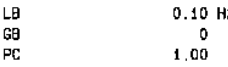

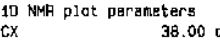

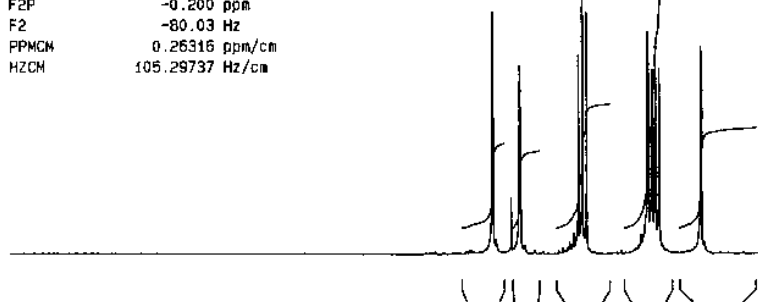

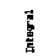

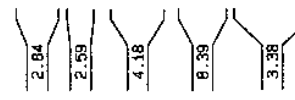

$\lim _{\operatorname{som}}$

(1)

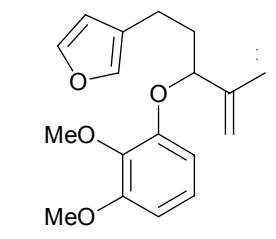


साज उसट

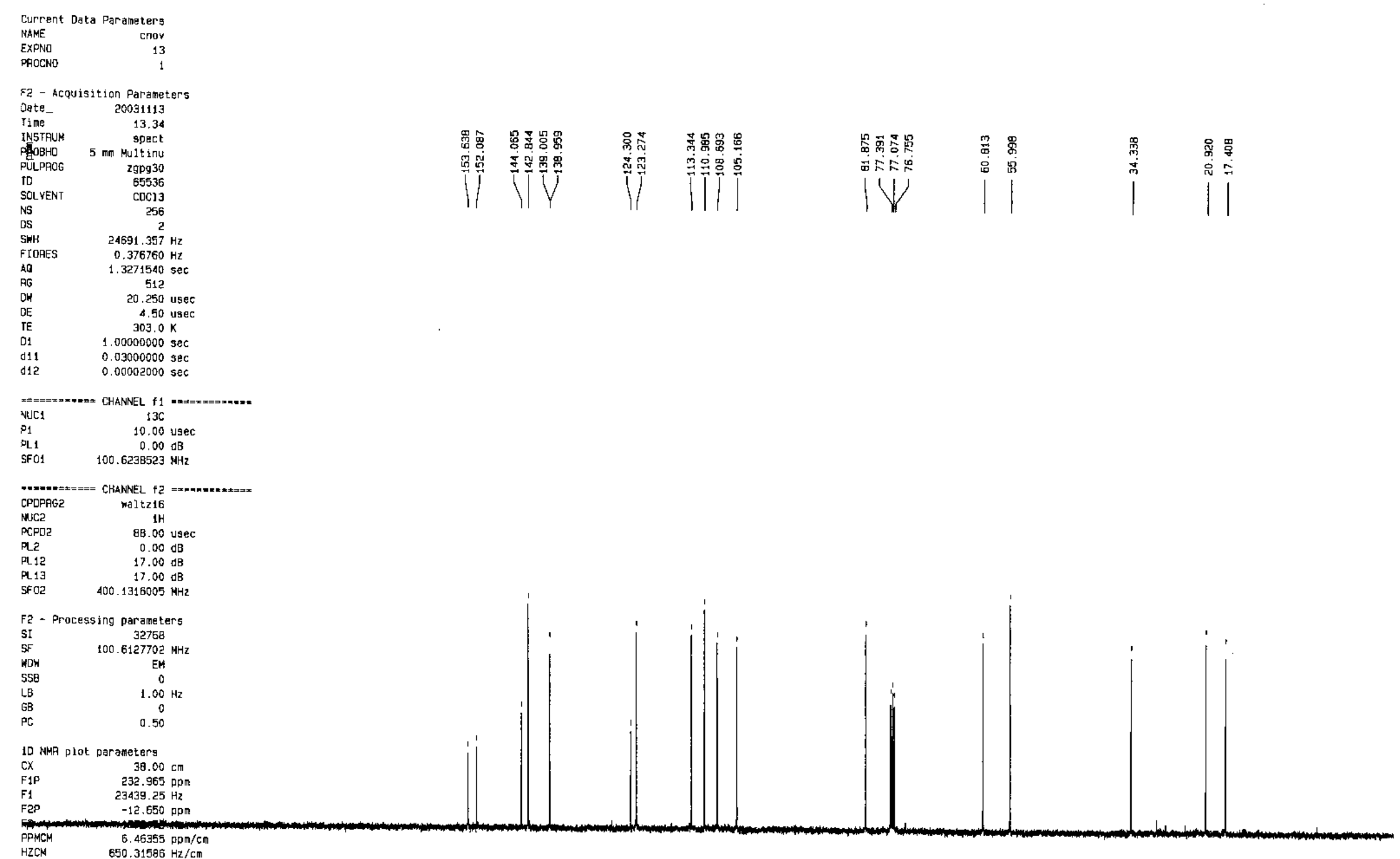

200

180

$\frac{1}{160}$

149

120

100

80

60

a 
6-[5-(3-Furyl)-2-methylpent-2-enyl]-2,3-dimethoxyphenol 24

4MJ 342

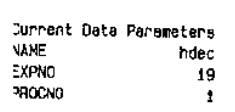

:2 - Acquisttion Parameters

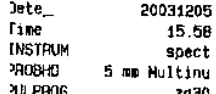

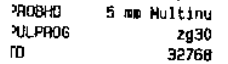

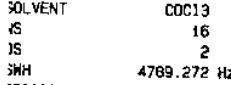

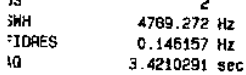

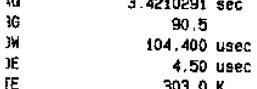

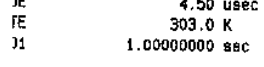

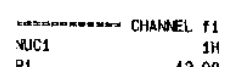

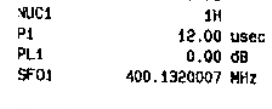

FI - Procoss ing par sheters

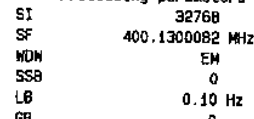

$\begin{array}{ll}L 6 & 0.10 \\ G B & 0 \\ \text { Ge } & 1.00\end{array}$

10 NWF plot parameters
CX
Fip $\quad 38.00 \mathrm{~cm}$

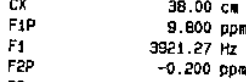

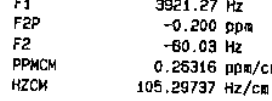

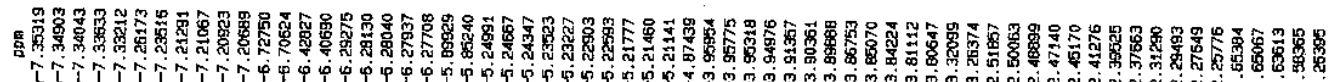

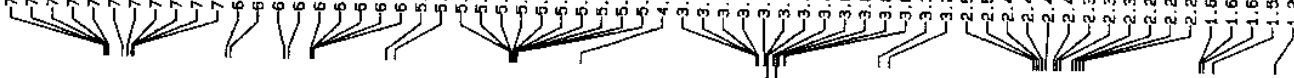

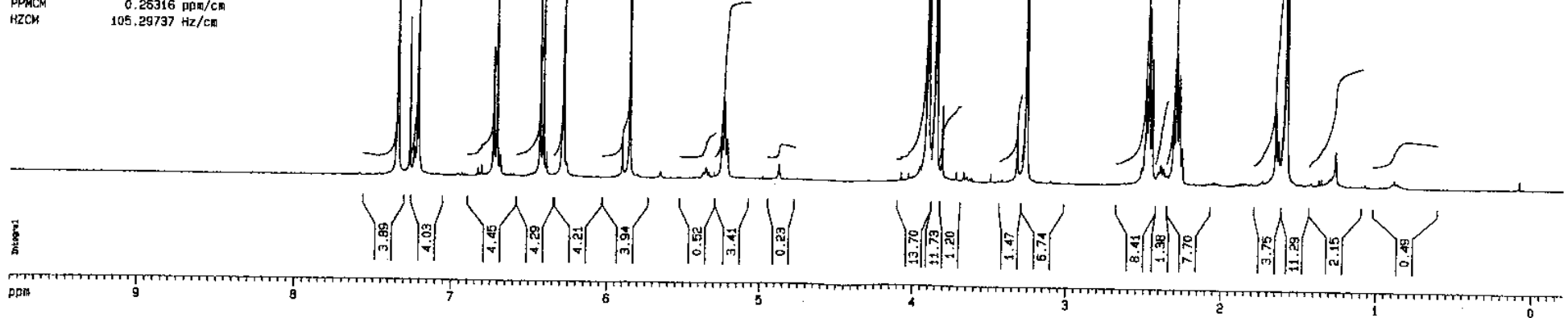


能的了程

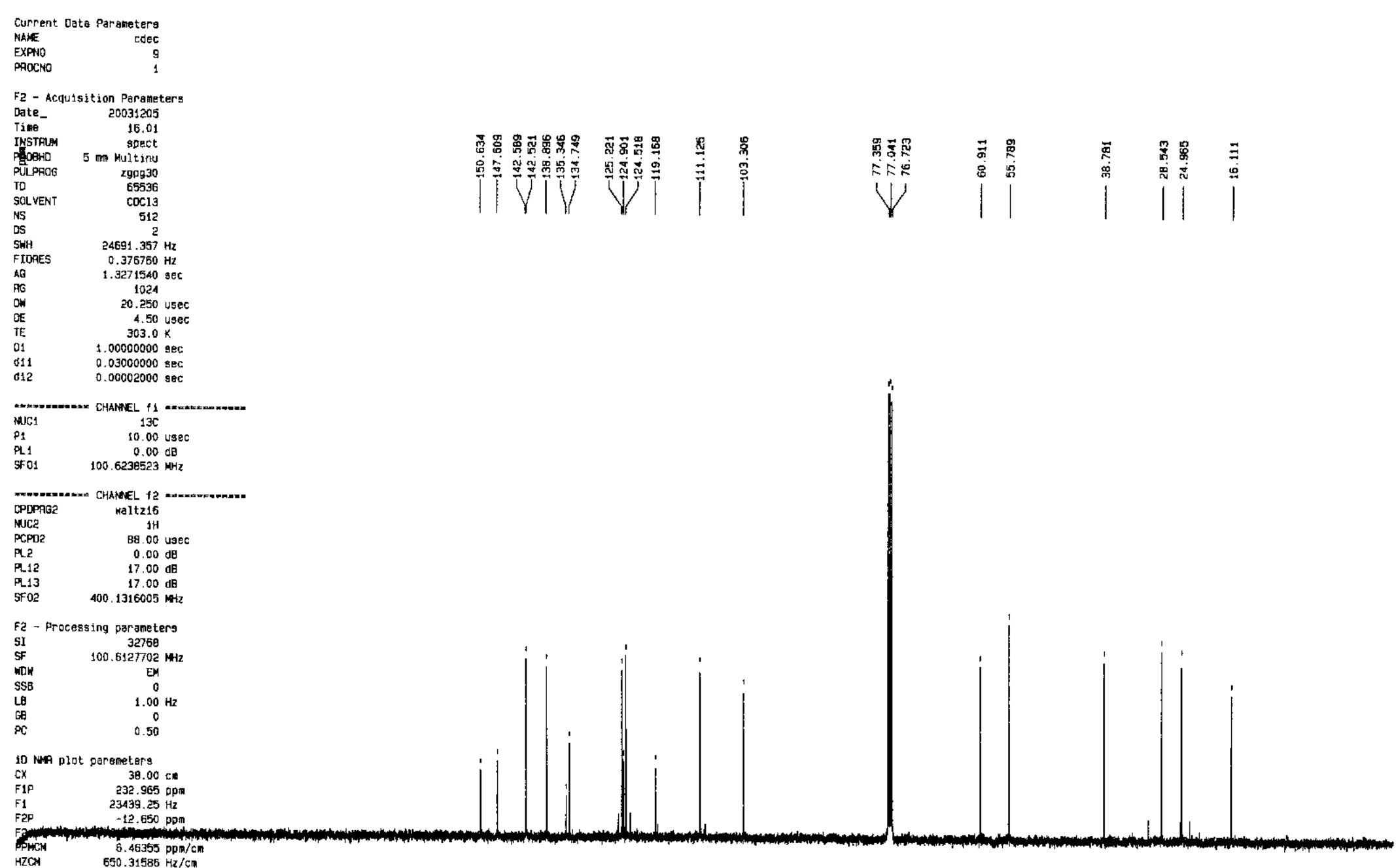

HZCA

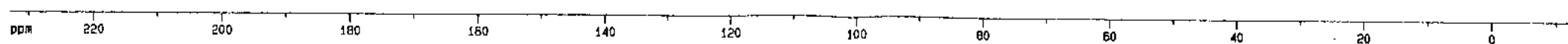


(E)-6-[5-(3-Furyl)-2-methylpent-2-enyl]-2,3-dimethoxy-1,4-benzoquinone (isoarnebifuranone) 9

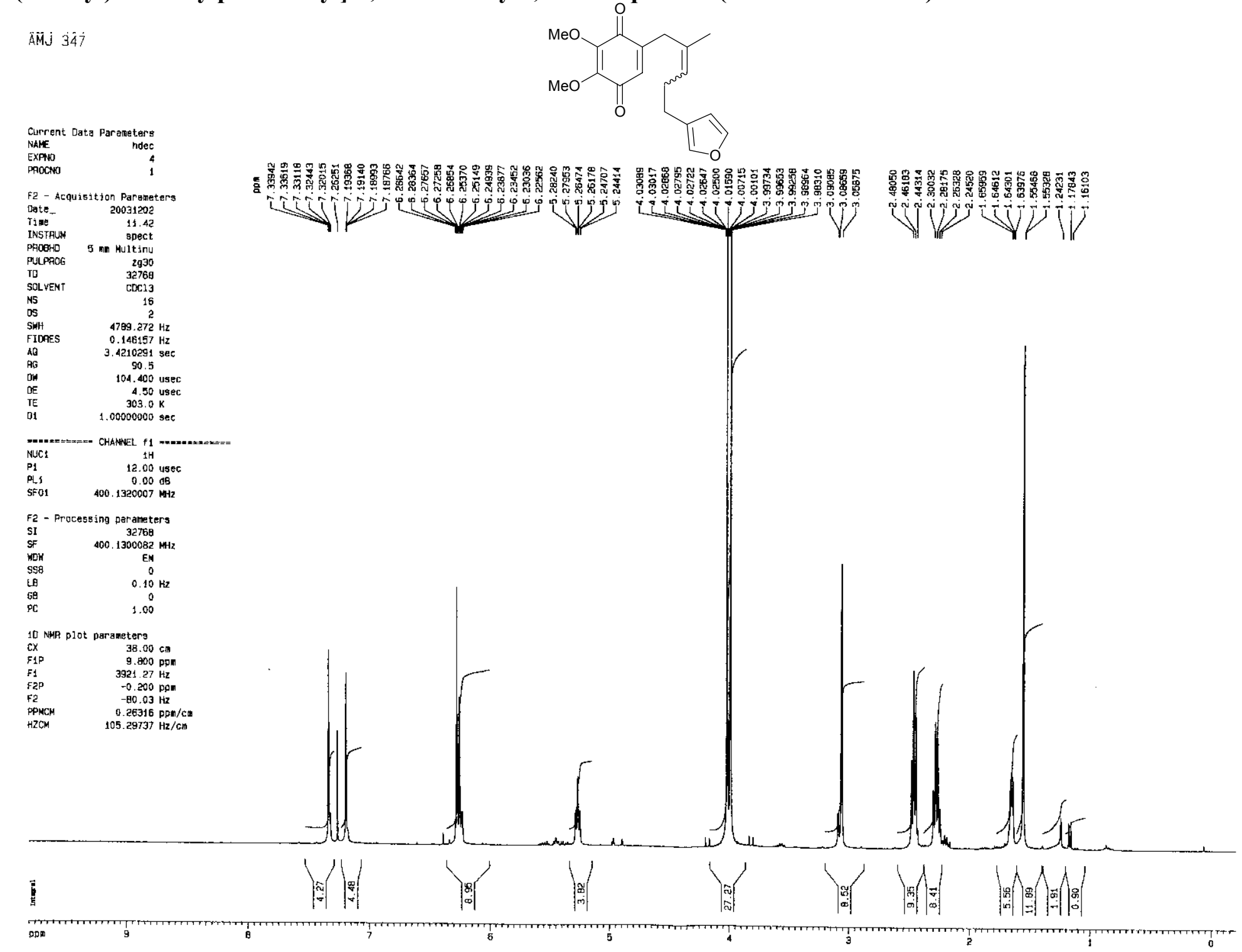



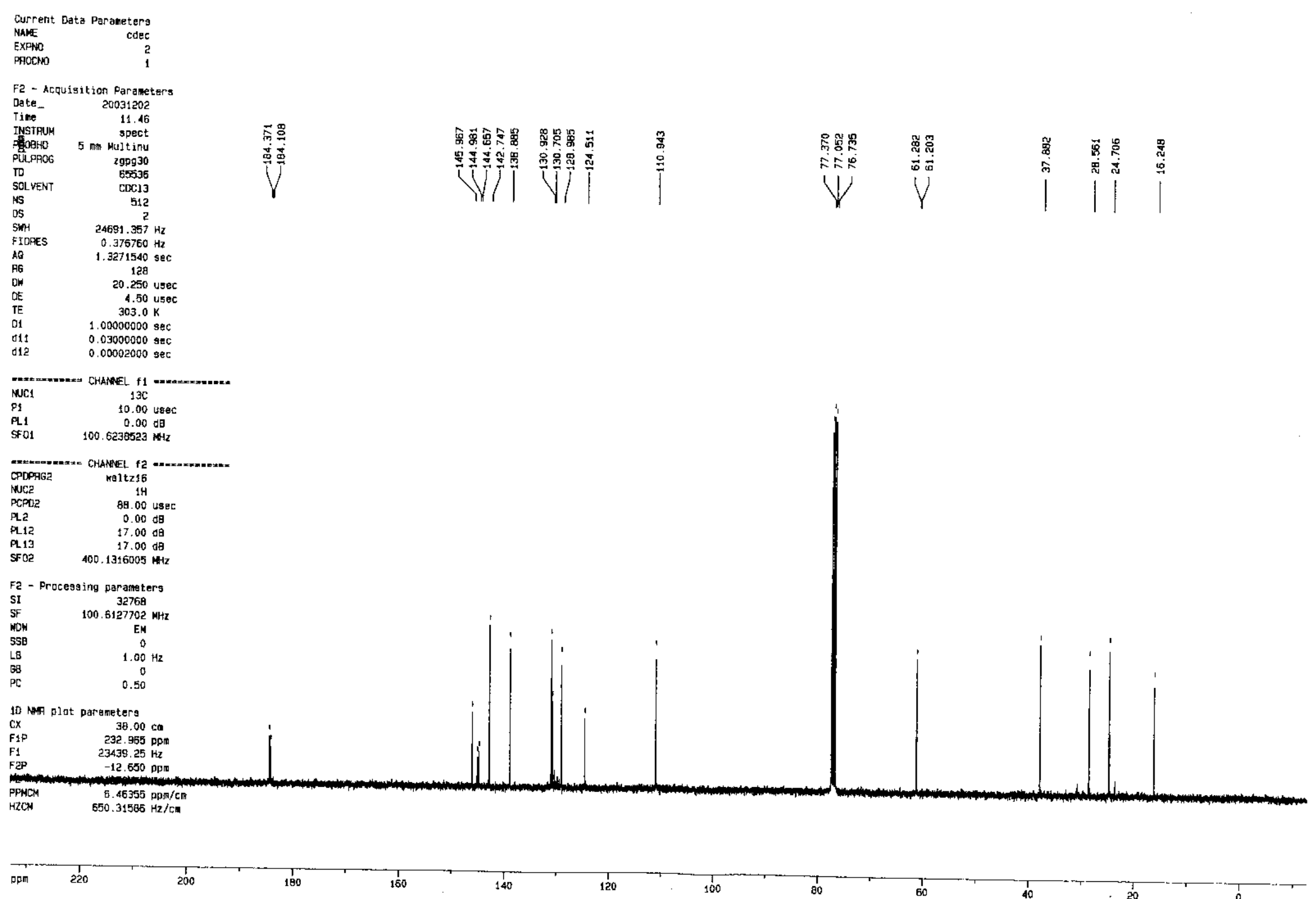


\section{Pentadec-1-en-3-ol}

To a solution of tridecanal (5.00 g, $25.2 \mathrm{mmol}, 1$ eq.) in anhydrous THF (50 mL) was added vinylmagnesium bromide (1 M in THF; $38 \mathrm{~mL}$, $38 \mathrm{mmol}, 1.5$ eq.) at $0{ }^{\circ} \mathrm{C}$ over $10 \mathrm{~min}$. The solution was stirred at $0{ }^{\circ} \mathrm{C}$ for $4 \mathrm{~h}$ under a nitrogen atmosphere. The reaction was quenched with saturated ammonium chloride $(60 \mathrm{~mL})$ and extracted with ethyl acetate $(3 \times 80 \mathrm{~mL})$. The combined organic layers were washed with water, dried $\left(\mathrm{MgSO}_{4}\right)$, filtered and the solvent was removed under reduced pressure. The crude product was purified by flash chromatography on silica, eluting with ethyl acetate-light petroleum (1:99) to give the title compound (4.14 g, 73\%) as a colorless oily solid (lit., ${ }^{1}(\mathrm{~S})$ enantiomer, no mp given), ${ }^{1} \mathrm{H}$ NMR (400 MHz; $\left.\mathrm{CDCl}_{3}\right) 5.87(1 \mathrm{H}, \mathrm{ddd}, J=17.2, J=10.3, J=6.2), 5.21(1 \mathrm{H}, \mathrm{d}, J=17.2), 5.09(1 \mathrm{H}, \mathrm{d}, J=$ 10.3), 4.20-4.00 (1H, m), 1.60-1.20 (22H, m), 0.88 (3H, t, $J=6.8) ;{ }^{13} \mathrm{C} \mathrm{NMR}\left(100 \mathrm{MHz}, \mathrm{CDCl}_{3}\right) 141.3(\mathrm{CH}), 114.5\left(\mathrm{CH}_{2}\right), 73.3(\mathrm{CH}), 37.0$ $\left(\mathrm{CH}_{2}\right), 31.9\left(\mathrm{CH}_{2}\right), 29.7-29.3\left(7 \times \mathrm{CH}_{2}\right), 25.3\left(\mathrm{CH}_{2}\right), 22.7\left(\mathrm{CH}_{2}\right), 14.1(\mathrm{Me}) ; \mathrm{MS}(\mathrm{CI}) 227\left(\mathrm{MH}^{+}, 67 \%\right), 225(100), 208(29), 207(60), 197$ (53), 165 (23), 111 (22), 97 (21); (Found: $\mathrm{MH}^{+}, 227.2386 . \mathrm{C}_{15} \mathrm{H}_{30} \mathrm{O}+\mathrm{H}$ requires 227.2375).

\section{Heptadec-1-en-3-ol}

To a solution of pentadecanal $(2.40 \mathrm{~g}, 10.6 \mathrm{mmol})$ in anhydrous THF $(20 \mathrm{~mL})$ was added vinylmagnesium bromide $(1 \mathrm{M}$ in THF; $16 \mathrm{~mL}, 16$ $\mathrm{mmol})$ at $0{ }^{\circ} \mathrm{C}$ over $10 \mathrm{~min}$. The solution was stirred at $0{ }^{\circ} \mathrm{C}$ for $2 \mathrm{~h}$ under a nitrogen atmosphere. The reaction was quenched with saturated ammonium chloride $(30 \mathrm{~mL})$ and extracted with ethyl acetate $(3 \times 40 \mathrm{~mL})$. The combined organic layers were washed with water $(3 \times 30$ $\mathrm{mL})$, brine $(30 \mathrm{~mL})$, dried $\left(\mathrm{MgSO}_{4}\right)$, filtered and evaporated in vacuo. The crude product was purified by flash chromatography on silica, eluting with ethyl acetate-light petroleum (1:9) to give the title compound (1.75 g, 65\%) as a colorless solid, mp $45-46{ }^{\circ} \mathrm{C}$ (from ethyl acetate-light petroleum) (lit., ${ }^{2}$ no data), IR (film)/ $\mathrm{cm}^{-1} 3366(\mathrm{O}-\mathrm{H}) ;{ }^{1} \mathrm{H} \mathrm{NMR}\left(300 \mathrm{MHz} ; \mathrm{CDCl}_{3}\right) 5.87(1 \mathrm{H}, \mathrm{td}, \mathrm{J}=6.2), 5.22(1 \mathrm{H}, \mathrm{dt}, \mathrm{J}=17.1$, 1.5), $5.10(1 \mathrm{H}, \mathrm{m}), 4.13-4.06(1 \mathrm{H}, \mathrm{m}), 1.59(1 \mathrm{H}, \mathrm{br}), 1.55-1.50(2 \mathrm{H}, \mathrm{m}), 1.26(24 \mathrm{H}, \mathrm{br}), 0.88(3 \mathrm{H}, \mathrm{t}, \mathrm{J}=7.0) ;{ }^{13} \mathrm{C} \mathrm{NMR}\left(75 \mathrm{MHz} ; \mathrm{CDCl}_{3}\right)$ 141.3 (CH), $114.5\left(\mathrm{CH}_{2}\right), 73.3(\mathrm{CH}), 37.1\left(\mathrm{CH}_{2}\right), 31.9\left(\mathrm{CH}_{2}\right), 29.7-29.4\left(9 \times \mathrm{CH}_{2}\right), 25.4\left(\mathrm{CH}_{2}\right), 22.7\left(\mathrm{CH}_{2}\right), 14.1(\mathrm{Me})$; $\mathrm{MS}(\mathrm{EI}) 254\left(\mathrm{M}^{+}\right.$, 3\%), 236 (94), 225 (84), 208 (21), 82 (21), 67 (29); (Found: $\mathrm{M}^{+}, 254.2621 . \mathrm{C}_{17} \mathrm{H}_{34} \mathrm{O}$ requires 254.2610). 


\section{3,7-Dimethyloct-6-en-1-yn-3-ol 13}

6-Methyl-5-hepten-2-one (4.68 mL, $4.0 \mathrm{~g}, 31.7 \mathrm{mmol})$ in anhydrous THF/ether $(60 \mathrm{~mL}, 1: 1)$ was cooled to $-10^{\circ} \mathrm{C}$. Ethynylmagnesium bromide (0.5 M in THF; $82.4 \mathrm{~mL}, 41.2 \mathrm{mmol}$ ) was added dropwise over a 10-20 min period while stirring below $0{ }^{\circ} \mathrm{C}$ under a nitrogen atmosphere. The mixture was then stirred for a further $2 \mathrm{~h}$ and then quenched with saturated ammonium chloride $(100 \mathrm{~mL})$, extracted with ethyl acetate $(3 \times 100 \mathrm{~mL})$, washed with water $(2 \times 100 \mathrm{~mL})$, brine $(100 \mathrm{~mL})$, dried $\left(\mathrm{MgSO}_{4}\right)$, filtered and evaporated in vacuo. The crude product was purified by flash chromatography on silica, eluting with ethyl acetate-light petroleum (1:19) to give the title compound $\mathbf{1 3}$ as a

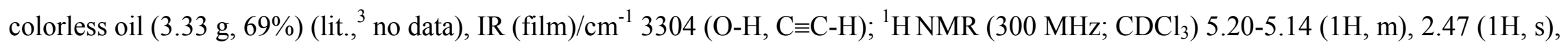
2.37-2.12 (2H, m), $2.05(1 \mathrm{H}, \mathrm{s}), 1.73-1.68(5 \mathrm{H}, \mathrm{m}), 1.66(3 \mathrm{H}, \mathrm{s}), 1.51(3 \mathrm{H}, \mathrm{s}) ;{ }^{13} \mathrm{C} \mathrm{NMR}\left(300 \mathrm{MHz} ; \mathrm{CDCl}_{3}\right) 133.1(\mathrm{C}), 124.0(\mathrm{CH}), 87.9(\mathrm{C})$, $71.9(\mathrm{CH}), 68.7(\mathrm{C}), 43.5\left(\mathrm{CH}_{2}\right), 30.2(\mathrm{Me}), 26.1(\mathrm{Me}), 24.0\left(\mathrm{CH}_{2}\right), 18.1(\mathrm{Me})$.

\section{5-(4-Methoxy-2,3,6-trimethylphenyl)-3-methylpentyn-3-ol 17}

To a solution of 4-(4-methoxy-2,3,6-trimethylphenyl)butan-2-one ${ }^{4}(2.50 \mathrm{~g}, 11.35 \mathrm{mmol})$ in THF/ether $(1: 1 ; 30 \mathrm{~mL})$ at $-5^{\circ} \mathrm{C}$ was added dropwise ethynylmagnesium bromide $(0.5 \mathrm{M}$ in THF; $27.24 \mathrm{~mL}, 13.62 \mathrm{mmol})$. The reaction mixture was stirred at between $-5{ }^{\circ} \mathrm{C}$ and $0{ }^{\circ} \mathrm{C}$ for $2 \mathrm{~h}$, then quenched with saturated ammonium chloride $(50 \mathrm{~mL})$. The aqueous phase was then extracted with ether $(3 \times 75 \mathrm{~mL})$. The combined ethereal extracts were washed with water $(2 \times 50 \mathrm{~mL})$ and brine $(50 \mathrm{~mL})$, dried $\left(\mathrm{MgSO}_{4}\right)$ and concentrated in vacuo. The crude product was purified by flash chromatography on silica gel, eluting with ethyl acetate-light petroleum (1:9) to afford the title compound $\mathbf{1 7}$

as a pale oil $\left(1.99 \mathrm{~g}, 71 \%\right.$ ), (lit., $\left.{ }^{4} \mathrm{mp} 55.5-57{ }^{\circ} \mathrm{C}\right)$, IR (film)/cm ${ }^{-1} 3584(\mathrm{O}-\mathrm{H}), 3305(\mathrm{C} \equiv \mathrm{C}-\mathrm{H}) ;{ }^{1} \mathrm{H} \mathrm{NMR}\left(300 \mathrm{MHz} ; \mathrm{CDCl}_{3}\right) 6.57(1 \mathrm{H}, \mathrm{s}), 3.79$ $(3 \mathrm{H}, \mathrm{s}), 2.88-2.81(2 \mathrm{H}, \mathrm{m}), 2.54(1 \mathrm{H}, \mathrm{s}), 2.45(3 \mathrm{H}, \mathrm{s}), 2.34(3 \mathrm{H}, \mathrm{s}), 2.14(3 \mathrm{H}, \mathrm{s}), 2.03(1 \mathrm{H}, \mathrm{s}), 1.80-1.73(2 \mathrm{H}, \mathrm{m}), 1.58(3 \mathrm{H}, \mathrm{s}) ;{ }^{13} \mathrm{C} \mathrm{NMR}(75$ MHz; $\left.\mathrm{CDCl}_{3}\right) 155.4(\mathrm{C}), 136.0(\mathrm{C}), 133.8(\mathrm{C}), 130.1(\mathrm{C}), 122.9(\mathrm{C}), 110.3(\mathrm{CH}), 87.3(\mathrm{C}), 71.7(\mathrm{CH}), 68.0(\mathrm{C}), 55.5(\mathrm{Me}), 42.8\left(\mathrm{CH}_{2}\right), 29.7$ (Me), $24.7\left(\mathrm{CH}_{2}\right), 20.3(\mathrm{Me}), 15.6(\mathrm{Me}), 12.0$ (Me); MS (EI) 246 (M+, 32\%), 191 (6), 177 (7), 163 (100), 97 (5); (Found: M+, 246.1626. $\mathrm{C}_{16} \mathrm{H}_{22} \mathrm{O}_{2}$ requires 246.1620). 


\section{(E)-Ethyl 3-(3-furyl)propenoate}

(Ethoxycarbonylmethylene)triphenylphosphorane $(20.14 \mathrm{~g}, 57.8 \mathrm{mmol})$ was added to a solution of 3-furaldehyde $(5.0 \mathrm{~mL}, 57.8 \mathrm{mmol}) \mathrm{in}$ dichloromethane $(70 \mathrm{~mL})$. The resulting mixture was heated under reflux and under a nitrogen atmosphere overnight. Then it was concentrated to one quarter of its original volume and light petroleum $(30 \mathrm{~mL})$ was added. The mixture was then filtered and washed through with light petroleum $(30 \mathrm{~mL})$. The solvents were removed in vacuo and the crude product was purified by flash chromatography on silica, eluting with ethyl acetate-light petroleum $(1: 19)$ to give the title compound $(8.94 \mathrm{~g}, 93 \%)$ as a colorless solid, mp $28-30{ }^{\circ} \mathrm{C}$ (from ethyl acetate-light petroleum) (lit., $\left.{ }^{5} \mathrm{mp} 38-38.5{ }^{\circ} \mathrm{C}\right) ; \mathrm{IR}(\mathrm{KBr}) / \mathrm{cm}^{-1} 1705(\mathrm{C}=\mathrm{O}) ;{ }^{1} \mathrm{H}$ NMR $\left(400 \mathrm{MHz} ; \mathrm{CDCl}_{3}\right) 7.64(1 \mathrm{H}, \mathrm{m}), 7.57(1 \mathrm{H}, \mathrm{d}, J=$ 15.8), $7.42(1 \mathrm{H}, \mathrm{s}), 6.58(1 \mathrm{H}, \mathrm{m}), 6.14(1 \mathrm{H}, \mathrm{d}, J=15.8), 4.23(2 \mathrm{H}, \mathrm{q}, J=7.2), 1.31(3 \mathrm{H}, \mathrm{t}, J=7.2) ;{ }^{13} \mathrm{C} \mathrm{NMR}\left(100 \mathrm{MHz} ; \mathrm{CDCl}_{3}\right) 167.0(\mathrm{C})$, $144.5(\mathrm{CH}), 144.4(\mathrm{CH}), 134.6(\mathrm{CH}), 122.6(\mathrm{C}), 117.9(\mathrm{CH}), 107.4(\mathrm{CH}), 60.4\left(\mathrm{CH}_{2}\right), 14.3(\mathrm{Me})$.

\section{Ethyl 3-(3-furyl)propanoate}

$\mathrm{Pd} / \mathrm{CaCO}_{3}(5 \% ; 0.873 \mathrm{~g})$ was added to a solution of ethyl 3-(3-furyl)propenoate (5.940 g, $\left.35.78 \mathrm{mmol}\right)$ in dry methanol (150 mL). The mixture was stirred under a hydrogen atmosphere for $1.5 \mathrm{~h}$. Then it was filtered through Celite, washed through with methanol ( $30 \mathrm{~mL})$. The solvent was removed under reduced pressure and the crude product was purified by flash chromatography on silica, eluting with ethyl acetate-light petroleum (1:49) to give the title compound (4.746 g, 79\%) as a colorless oil (lit., ${ }^{6}$ bp $95{ }^{\circ} \mathrm{C} / 3 \mathrm{mmHg}$ ), IR (film) $/ \mathrm{cm}^{-1} 1736$ $(\mathrm{C}=\mathrm{O}) ;{ }^{1} \mathrm{H}$ NMR $\left(400 \mathrm{MHz} ; \mathrm{CDCl}_{3}\right)$ 7.34-7.33 (1H, m), 7.23-7.22 (1H, m, ), 6.27 (1H, s), $4.13(2 \mathrm{H}, \mathrm{q}, J=7.2), 2.8-2.7(2 \mathrm{H}, \mathrm{m}), 2.6-2.5(2 \mathrm{H}$, m), $1.24(3 \mathrm{H}, \mathrm{t}, J=7.2) ;{ }^{13} \mathrm{C} \mathrm{NMR}\left(100 \mathrm{MHz} ; \mathrm{CDCl}_{3}\right) 172.9(\mathrm{C}), 142.0(\mathrm{CH}), 139.0(\mathrm{CH}), 123.5(\mathrm{C}), 110.8(\mathrm{CH}), 60.5\left(\mathrm{CH}_{2}\right), 34.8\left(\mathrm{CH}_{2}\right)$, $20.3\left(\mathrm{CH}_{2}\right), 14.2(\mathrm{Me})$. 


\section{3-(3-Furyl)propanal 21}

To a solution of ethyl 3-(3-furyl)propanoate $(8.711 \mathrm{~g}, 51.85 \mathrm{mmol})$ in ether $(260 \mathrm{~mL})$ at $-78{ }^{\circ} \mathrm{C}$ was added diisobutylaluminium hydride $(1 \mathrm{M}$ in dichloromethane; $57 \mathrm{~mL}, 57.04 \mathrm{mmol}$ ) so that the temperature was maintained below $-70{ }^{\circ} \mathrm{C}$. The resulting solution was stirred for a further $45 \mathrm{~min}$ at $-70{ }^{\circ} \mathrm{C}$. Then it was quenched with saturated ammonium chloride $(50 \mathrm{~mL})$, filtered and washed through with ether $(300$ $\mathrm{mL})$. The organic layer was dried $\left(\mathrm{MgSO}_{4}\right)$ and the solvents were removed in vacuo. The residue was distilled under reduced pressure to give the title compound 21 (4.347 g, 68\%) as a colorless oil, bp 100-150 ${ }^{\circ} \mathrm{C} / \mathrm{ca} .12 \mathrm{mmHg}\left(\right.$ lit., $\left.{ }^{6} \mathrm{bp} 90-95{ }^{\circ} \mathrm{C} / 10 \mathrm{mmHg}\right), \mathrm{IR}\left(\right.$ film) $/ \mathrm{cm}^{-1} 2724$ $(\mathrm{C}-\mathrm{H}), 1726(\mathrm{C}=\mathrm{O}) ;{ }^{1} \mathrm{H}$ NMR $\left(400 \mathrm{MHz} ; \mathrm{CDCl}_{3}\right) 9.81(1 \mathrm{H}, \mathrm{s}), 7.35(1 \mathrm{H}, \mathrm{s}), 7.23(1 \mathrm{H}, \mathrm{s}), 6.26(1 \mathrm{H}, \mathrm{s}), 2.82-2.67(4 \mathrm{H}, \mathrm{m}) ;{ }^{13} \mathrm{C} \mathrm{NMR}(100$ MHz; $\left.\mathrm{CDCl}_{3}\right) 201.7(\mathrm{CH}), 143.1(\mathrm{CH}), 139.1(\mathrm{CH}), 123.3(\mathrm{C}), 110.8(\mathrm{CH}), 44.0\left(\mathrm{CH}_{2}\right), 17.5\left(\mathrm{CH}_{2}\right)$; $\mathrm{MS}(\mathrm{CI}) 125\left(\mathrm{MH}^{+}, 100 \%\right), 109(27)$, 107 (17), 81 (16); (Found: $\mathrm{MH}^{+}, 125.0597 . \mathrm{C}_{7} \mathrm{H}_{8} \mathrm{O}_{2}+\mathrm{H}$ requires 125.0603).

\section{5-(3-Furyl)-2-methylpenten-3-ol 22}

To a solution of 3-(3-furyl)propanal 21 (3.566 g, $28.76 \mathrm{mmol})$ in ether $(140 \mathrm{~mL})$ at $-10{ }^{\circ} \mathrm{C}$ was added isopropenylmagnesium bromide $(0.5$ $\mathrm{M}$ in THF; $86.3 \mathrm{~mL}, 43.14 \mathrm{mmol})$ and the resulting solution was stirred for a further $4 \mathrm{~h}$ between -10 and $0{ }^{\circ} \mathrm{C}$. The solution was quenched with saturated ammonium chloride $(50 \mathrm{~mL})$ and extracted with ether. The combined organic extracts were dried $\left(\mathrm{MgSO}_{4}\right)$, filtered and the solvent was removed in vacuo. The crude product was purified by flash chromatography on silica, eluting with ether and light petroleum (1:19) to give the title compound $22(3.306 \mathrm{~g}, 69 \%)$ as a pale yellow oil (lit., ${ }^{7}$ no bp given), IR (film)/ $\mathrm{cm}^{-1} 3370(\mathrm{O}-\mathrm{H})$; ${ }^{1} \mathrm{H} \mathrm{NMR}(400 \mathrm{MHz}$; $\left.\mathrm{CDCl}_{3}\right) 7.34(1 \mathrm{H}, \mathrm{s}), 7.22(1 \mathrm{H}, \mathrm{s}), 6.27(1 \mathrm{H}, \mathrm{s}), 4.95(1 \mathrm{H}, \mathrm{s}), 4.86(1 \mathrm{H}, \mathrm{s}), 4.07(1 \mathrm{H}, \mathrm{t}, J=6.4), 2.57-2.38(2 \mathrm{H}, \mathrm{m}), 2.06-1.95(1 \mathrm{H}, \mathrm{m}), 2.03-$ $1.97(1 \mathrm{H}, \mathrm{br}), 1.85-1.75(1 \mathrm{H}, \mathrm{m}), 1.73(3 \mathrm{H}, \mathrm{s}) ;{ }^{13} \mathrm{C} \mathrm{NMR}\left(100 \mathrm{MHz}, \mathrm{CDCl}_{3}\right) 147.4(\mathrm{C}), 142.8(\mathrm{CH}), 138.9(\mathrm{CH}), 124.6(\mathrm{C}), 112.2\left(\mathrm{CH}_{2}\right)$, $110.9(\mathrm{CH}), 75.2(\mathrm{CH}), 35.0\left(\mathrm{CH}_{2}\right), 20.9\left(\mathrm{CH}_{2}\right), 17.5(\mathrm{Me})$; MS (CI) $167\left(\mathrm{MH}^{+}, 14 \%\right), 166\left(\mathrm{M}^{+}, 18\right), 149(91), 1319(25), 121(23), 109$ (30), 95 (25), 82 (30), 81 (100); (Found: $\mathrm{MH}^{+}, 167.1068 . \mathrm{C}_{10} \mathrm{H}_{14} \mathrm{O}_{2}+\mathrm{H}$ requires 167.1072).

\section{References}

(1) Dorta, R. L.; Rodriquez, M. S.; Salazar, J. A.; Suarez, E. Tetrahedron Lett. 1997, 38, 4675-4678.

(2) Saito, A.; Saito, K.; Tanaka, A.; Oritani, T. Tetrahedron Lett. 1997, 38, 3955-3958. 
(3) Kahn, P. H.; Cossy, J. Tetrahedron Lett. 1999, 40, 8113-8114.

(4) Samokhvalov, G. I.; Zakharova, N. I.; Sokolova, N. N.; Filippova, T. M.; Raigorodskaya, O. I.; Murav'ev, V. V.; Tuguzova, A. M.; Khristoforov, V. L. Pharm. Chem. J. 1994, 28, 343-348.

(5) Crimmins, M. T.; Jung, D. K.; Gray, J. L. J. Am. Chem. Soc. 1993, 115, 3146-3155.

(6) Weyerstahl, P.; Schenk, A.; Marschall, H. Liebigs Ann. 1995, 1849-1853.

(7) Foland, L. D.; Decker, O. H. W.; Moore, H. W. J. Am. Chem. Soc. 1989, 111, 989-995. 